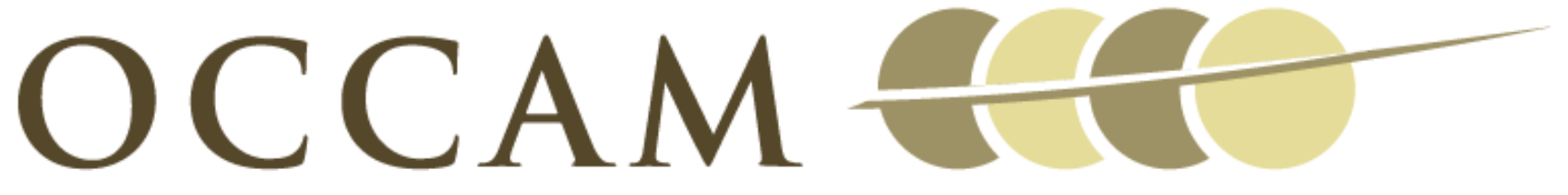

OXFORD CENTRE FOR COLLABORATIVE APPLIED MATHEMATICS

Report Number 10/13

Nonlinear Morphoelastic Plates II: Exodus to Buckled States

by

Joseph McMahon, Alain Goriely, and Michael Tabor

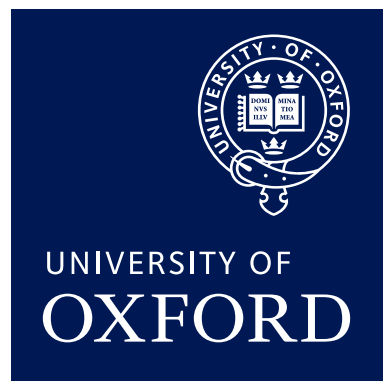

Oxford Centre for Collaborative Applied Mathematics Mathematical Institute 24 - 29 St Giles'

Oxford

OX1 3LB

England 



\section{Nonlinear Morphoelastic Plates II: Exodus to Buckled States}

\section{Joseph McMahon*, Alain Goriely*i‡, and Michael Tabor ${ }^{* \dagger}$}

${ }^{*}$ Program in Applied Mathematics and ${ }^{\dagger}$ Department of Mathematics, University of Arizona, Building \#89, Tucson, AZ 85721

${ }^{\dagger}$ OCCAM, Institute of Mathematics, University of Oxford, UK;

email: goriely@maths.ox.ac.uk, Corresponding author: A. G.

Abstract Morphoelasticity is the theory of growing elastic materials. This theory is based on the multiple decomposition of the deformation gradient and provides a formulation of the deformation and stresses induced by growth. Following a companion paper, a general theory of growing nonlinear elastic Kirchhoff plate is described. First, a complete geometric description of incompatibility with simple examples is given. Second, the stability of growing Kirchhoff plates is analyzed.

KEYWORDS: Nonlinear elasticity, Kirchhoff Plates, Growth, Residual Stress, Buckling.

\section{Contents}

1 Introduction $\quad 2$

2 Geometry and Kinematics of Deformation and Growth 3

2.1 Coordinates and Euclidean Space $\mathbb{E}^{3} \ldots \ldots \ldots$

2.2 Manifold with Cylindrical Coordinates . . . . . . . . . . . . . . . . . 3

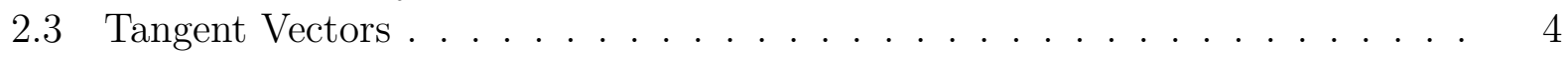

2.3.1 Tangent Vectors in $\mathbb{E}^{3} \ldots \ldots \ldots \ldots$. . . . . . . . . . 4

2.3 .2 Tangent Vectors in $\mathbb{R}^{3} \ldots \ldots \ldots \ldots$

2.4 Metric Tensor . . . . . . . . . . . . . . . . . . . 6

2.4.1 Deformation and Change of Metric Tensor . . . . . . . . . . . . . 7

2.5 Incompatible Growth . . . . . . . . . . . . . . . . . . . . . . . . . . 10

2.6 Immersibility and Embeddability . . . . . . . . . . . . . . . . 10

2.6.1 Immersions, Embeddings, and Isometry . . . . . . . . . . . . . . . 10

2.6.2 Riemann-Christoffel Curvature Tensor . . . . . . . . . . . . . . . 11

2.7 Example: Immersion without Embedding . . . . . . . . . . . . . . . . 12

2.8 Lack of Immersion . . . . . . . . . . . . . . . . . . . . . . . . . . . . . . . . . . . . . . . . . . . . . . . .

2.9 Non-Metric Complications . . . . . . . . . . . . . . . . . . 15

3 Deformation and Hyperelasticity 16

3.1 The Multiplicative Decomposition . . . . . . . . . . . . . . . 16

3.2 Constitutive Relation of an Elastic Body with Growth . . . . . . . . . . . . 17

3.2.1 Traditional Hyperelasticity . . . . . . . . . . . . . . . . . . . 17

3.2.2 Hyperelasticity with Growth . . . . . . . . . . . . . 18 
5 The Kirchhoff Plate $\quad 19$

5.1 Kirchhoff Constraints . . . . . . . . . . . . . . . . . . . . 19

5.2 Kinematics of an Axisymmetric Kirchhoff Plate . . . . . . . . . . . . 21

5.3 Addressing Lagrange Multipliers . . . . . . . . . . . . . . . . . . . . . 27

6 Formulation of the problem as a system of ODEs 30

7 Applying the Constitutive Relation 33

7.1 Without Growth . . . . . . . . . . . . . . . . 33

7.2 With Growth . . . . . . . . . . . . . . . . . 34

8 Stability of the Flat Plate $\quad 35$

8.1 The Flat Plate . . . . . . . . . . . . . . . . . . . . . 35

8.2 Differential Equations for Bifurcation . . . . . . . . . . . . . . . 36

8.3 Linear Boundary Conditions for Bifurcation . . . . . . . . . . . . . 39

8.4 Evidence of Bifurcation . . . . . . . . . . . . . . . . . . . . . 39

8.5 Numerical Results . . . . . . . . . . . . . . . . . . . . 41

9 Large Buckling Due to Immersibility-Precluding Growth 42

10 Conclusion $\quad 43$

\section{Introduction}

In a companion paper [12] we demonstrated that residual stress can be generated through growth in a nonlinear Kirchhoff plate. It has been established that under appropriate circumstances, residual stress induced through growth can be sufficient to create instabilities in an elastic three-dimensional material $[9,4]$. It is therefore a natural question to investigate whether such stress fields can be sufficient to initiate buckling in plates. Surprisingly, the case of two-dimensional structures has not been analyzed within the framework of nonlinear elasticity (note however, a growing body of literature in the physics community-all related to extension of the Föppl-von Kármán equation to include the effect of growth through extra curvature terms [8, 7], see [12] for a general introduction and citations). Here, we combine incompatible growth with the global constraint principle ([2], [10]) that has clarified and simplified the theory of finite deformations of rods and shells, geometrically exact models can be constructed for a wide variety of shapes. We demonstrate that such theories can be easily adapted to include growth. Further, we solve explicit examples of plate models and find residual stress and buckling caused by the elastic response to growth. Whereas, the notation and basic theory follows the discussion introduced in [12], we further analyze the general theory of incompatibility based on differential geometry before analysing the buckling problem.

The structure of the paper is as follows: In Section 2 we review the basic kinematics of finite deformations of solids and show its relation to the geometry of differentiable manifolds. 
Using the notation and tools of kinematics and differential geometry, we construct concrete examples of continuously differentiable two-point tensor fields with positive determinant that are not deformation gradients. We expose three distinct ways in which such tensor fields can fail the requirements of a deformation gradient. Such tensor fields are called incompatible growth fields.

In Section 3 we introduce the multiplicative decomposition of the deformation gradient into growth and elastic components. The proper method for incorporating the multiplicative decomposition into constitutive relations is shown.

Section 4 introduces the global constraint principle by which rod and shell theories are derived. In Section 5 we derive the kinematics of a buckled axisymmetric Kirchhoff plate, find the virtual displacements from such a configuration, and apply the global constraint principle to derive balance equations, which are simplified in Section 6 . In Section 7 we introduce a nonlinearly elastic constitutive relation, modify it with the multiplicative decomposition of the deformation gradient, and import the result into the balance equations.

Section 8 explores the appearance of buckled solutions alongside un-buckled configurations. We find the impact of thickness on the onset of buckling in response to incompatible growth. In Section 9 we find large buckling in another example of elastic response to growth.

\section{Geometry and Kinematics of Deformation and Growth}

\subsection{Coordinates and Euclidean Space $\mathbb{E}^{3}$}

The space in which a solid body resides is three-dimensional Euclidean space $\mathbb{E}^{3}$, which consists of (spatial) points, each of which has an inner-product space of tangent vectors attached to it. $\mathbb{E}^{3}$ has a fixed right-handed trio of orthogonal Cartesian axes, and each tangent space has unit vectors $\boldsymbol{i}, \boldsymbol{j}$, and $\boldsymbol{k}$ pointing along these axes. We will also use

cylindrical coordinates $(R, \Theta, Z)$. The unit tangent vectors $\boldsymbol{h}_{1}, \boldsymbol{h}_{2}$, and $\boldsymbol{k}$ point in the directions of increasing $R, \Theta$, and $Z$, respectively. They are related to the Cartesian tangent vectors by

$$
\begin{aligned}
\boldsymbol{h}_{1}(\Theta) & =\cos \Theta \boldsymbol{i}+\sin \Theta \boldsymbol{j}, \\
\boldsymbol{h}_{2}(\Theta) & =-\sin \Theta \boldsymbol{i}+\cos \Theta \boldsymbol{j} .
\end{aligned}
$$

Although $\mathbb{E}^{3}$ consists of spatial points, in most cases we will refer to a point not by its coordinates, but by the tangent vector anchored at the origin that points to the point.

\subsection{Manifold with Cylindrical Coordinates}

We will treat a solid body as a differentiable manifold with boundary, which is a set of material points that can be assigned coordinates in an invertible manner, at least locally. In the case at hand, we consider a multiply connected manifold $\mathcal{B}$ that is assigned cylindrical coordinates $(R, \Theta, Z)$. We may need to employ different branches of the azimuthal angle $\Theta$ for situations such as those illustrated in Figures 1 and 2 . The map $\Psi$ from $\mathcal{B}$ to coordinate 
points is called a coordinate system, and $\Psi(\mathcal{B})$ will be called the space of coordinates.

Now that the body $\mathcal{B}$ is represented by a collection of coordinate points, we define the position function $\widehat{\boldsymbol{X}}: \Psi(\mathcal{B}) \rightarrow \mathbb{E}^{3}$ by

$$
\widehat{\boldsymbol{X}}(R, \Theta, Z)=R \cos \Theta \boldsymbol{i}+R \sin \Theta \boldsymbol{j}+Z \boldsymbol{k}=R \boldsymbol{h}_{1}(\Theta)+Z \boldsymbol{k} .
$$

The map $\widehat{\boldsymbol{X}} \circ \Psi$ takes each point $p \in \mathcal{B}$ and assigns it a position $(\widehat{\boldsymbol{X}} \circ \Psi)(p)=\widehat{\boldsymbol{X}}(R(p), \Theta(p), Z(p))$ in $\mathbb{E}^{3}$ in an invertible manner. The set $(\widehat{\boldsymbol{X}} \circ \Psi)(\mathcal{B})$ will often be called the "Euclidean body". Note that the position function $\widehat{\boldsymbol{X}}$ maps to the same point in $\mathbb{E}^{3}$ regardless of whether the chart in Figure 1 or the chart in Figure 2 is used.

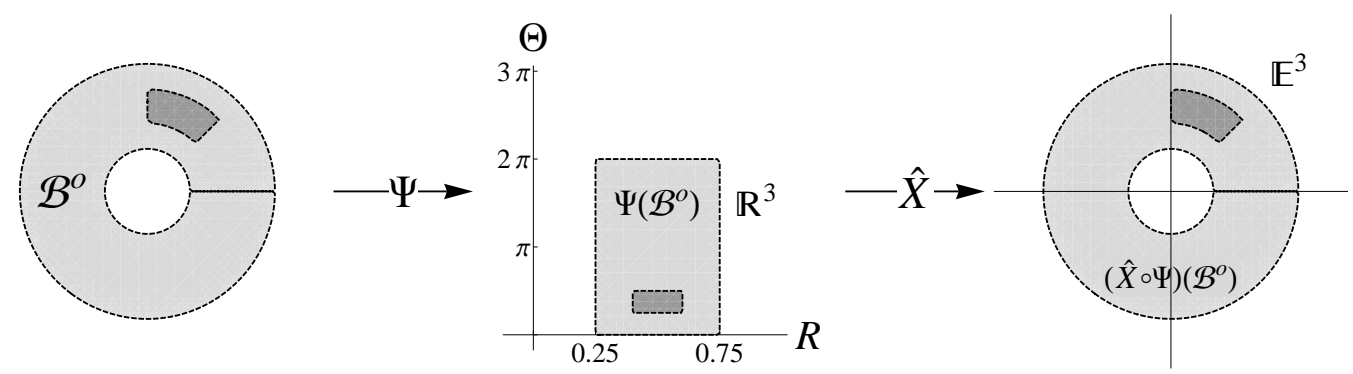

Figure 1: One chart for cylindrical coordinates assigns $\Theta$-coordinates between 0 and $2 \pi$. The position function $\widehat{\boldsymbol{X}}$ for this chart sends this portion of $\mathbb{R}^{3}$ into $\mathbb{E}^{3}$.

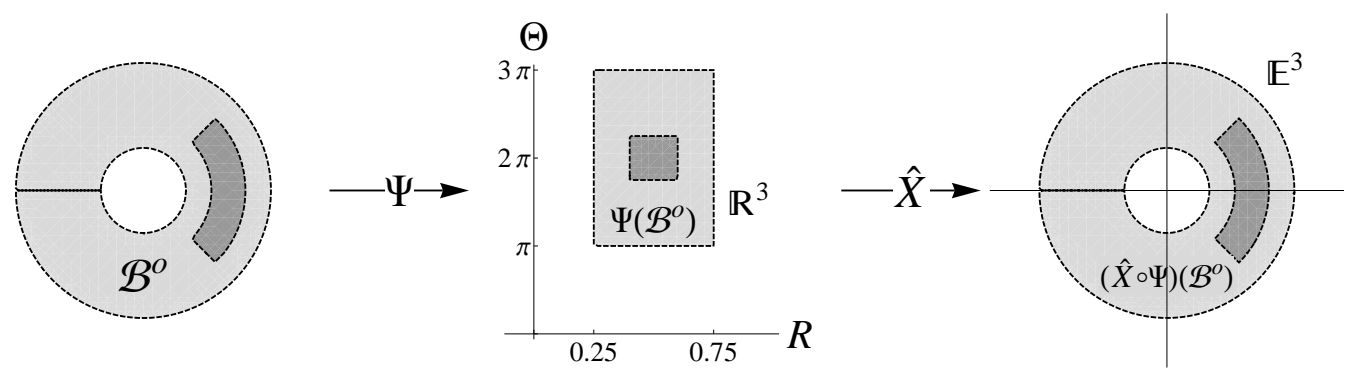

Figure 2: Some subsets of $\mathcal{B}^{\circ}$ (the open interior of $\mathcal{B}$ ) may straddle the line that corresponds to $\Theta=0=2 \pi$ in the chart in Figure 1. To avoid a disconnected image of the subset in $\mathbb{R}^{3}$, we choose a different chart, which assigns $\Theta$-coordinates between $\pi$ and $3 \pi$. The position function $\widehat{\boldsymbol{X}}$ for this chart sends this portion of $\mathbb{R}^{3}$ into $\mathbb{E}^{3}$.

\subsection{Tangent Vectors}

\subsubsection{Tangent Vectors in $\mathbb{E}^{3}$}

To each point $\boldsymbol{X}$ in the Euclidean body there is attached a three-dimensional real vector space whose elements are called tangent vectors. At $\boldsymbol{X}$ a basis for this tangent space is 
provided by the three partial derivatives of the position function $\widehat{\boldsymbol{X}}$ :

$$
\begin{aligned}
& \boldsymbol{E}_{R}=\frac{\partial \widehat{\boldsymbol{X}}}{\partial R}=\cos \Theta \boldsymbol{i}+\sin \Theta \boldsymbol{j}, \\
& \boldsymbol{E}_{\Theta}=\frac{\partial \widehat{\boldsymbol{X}}}{\partial \Theta}=-R \sin \Theta \boldsymbol{i}+R \cos \Theta \boldsymbol{j}, \\
& \boldsymbol{E}_{Z}=\frac{\partial \widehat{\boldsymbol{X}}}{\partial Z}=\boldsymbol{k} .
\end{aligned}
$$

This basis is called the coordinate basis. There is a corresponding dual basis of vectors $\boldsymbol{E}^{j}$ defined so that $\boldsymbol{E}^{j} \cdot \boldsymbol{E}_{i}=\delta_{i}^{j}$ :

$$
\begin{aligned}
& \boldsymbol{E}^{R}=\cos \Theta \boldsymbol{i}+\sin \Theta \boldsymbol{j}, \\
& \boldsymbol{E}^{\Theta}=-R^{-1} \sin \Theta \boldsymbol{i}+R^{-1} \cos \Theta \boldsymbol{j}, \\
& \boldsymbol{E}^{Z}=\boldsymbol{k} .
\end{aligned}
$$

The inner products $E_{i j}=\boldsymbol{E}_{i} \cdot \boldsymbol{E}_{j}$ form a symmetric, positive-definite matrix:

$$
\left(\begin{array}{ccc}
E_{R R} & E_{R \Theta} & E_{R Z} \\
E_{\Theta R} & E_{\Theta \Theta} & E_{\Theta Z} \\
E_{Z R} & E_{Z \Theta} & E_{Z Z}
\end{array}\right)=\left(\begin{array}{ccc}
1 & 0 & 0 \\
0 & R^{2} & 0 \\
0 & 0 & 1
\end{array}\right) ;
$$

while the inner products $E^{i j}=\boldsymbol{E}^{i} \cdot \boldsymbol{E}^{j}$ form the inverse matrix:

$$
\left(\begin{array}{ccc}
E^{R R} & E^{R \Theta} & E^{R Z} \\
E^{\Theta R} & E^{\Theta \Theta} & E^{\Theta Z} \\
E^{Z R} & E^{Z \Theta} & E^{Z Z}
\end{array}\right)=\left(\begin{array}{ccc}
1 & 0 & 0 \\
0 & R^{-2} & 0 \\
0 & 0 & 1
\end{array}\right)
$$

\subsubsection{Tangent Vectors in $\mathbb{R}^{3}$}
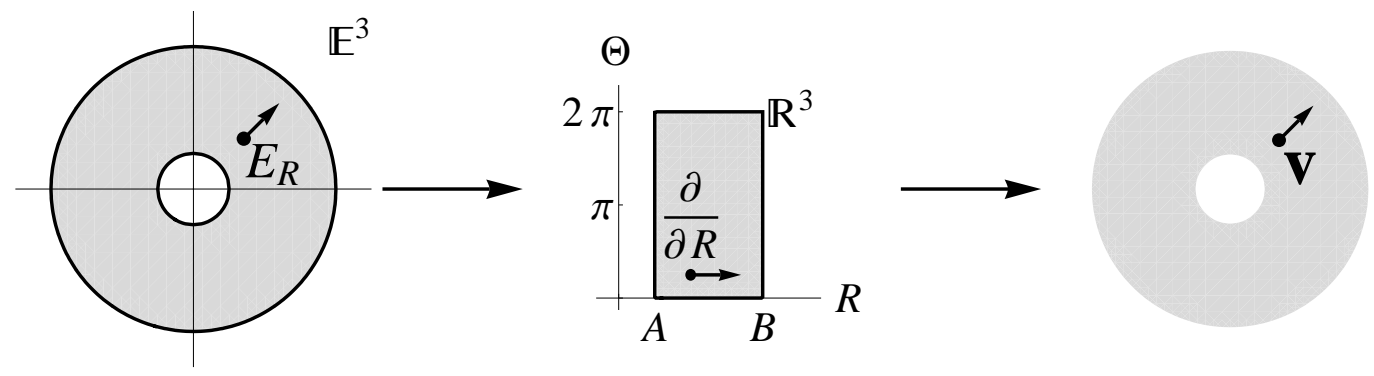

Figure 3: Each tangent vector at a point $\widehat{\boldsymbol{X}}(R, \Theta, Z)$ in the Euclidean body corresponds to a unique tangent vector at $(R, \Theta, Z) \in \Psi(\mathcal{B})$ and to a unique abstract tangent vector at $\Psi^{-1}(R, \Theta, Z) \in \mathcal{B}$.

For each tangent vector at a point $\widehat{\boldsymbol{X}}(R, \Theta, Z)$ in the Euclidean body there is a unique tangent vector at $(R, \Theta, Z) \in \Psi(\mathcal{B})$. In the space of coordinates, the tangent vectors are 
represented by partial differential operators. The correspondence is

$$
\begin{aligned}
\boldsymbol{E}_{R}=\frac{\partial \widehat{\boldsymbol{X}}}{\partial R} \quad \text { at } \widehat{\boldsymbol{X}}(R, \Theta, Z) \longleftrightarrow \frac{\partial}{\partial R} \quad \text { at }(R, \Theta, Z), \\
\boldsymbol{E}_{\Theta}=\frac{\partial \widehat{\boldsymbol{X}}}{\partial \Theta} \quad \text { at } \widehat{\boldsymbol{X}}(R, \Theta, Z) \longleftrightarrow \frac{\partial}{\partial \Theta} \quad \text { at }(R, \Theta, Z), \\
\boldsymbol{E}_{Z}=\frac{\partial \widehat{\boldsymbol{X}}}{\partial Z} \quad \text { at } \widehat{\boldsymbol{X}}(R, \Theta, Z) \longleftrightarrow \frac{\partial}{\partial Z} \quad \text { at }(R, \Theta, Z) .
\end{aligned}
$$

There is also a one-to-one correspondence between the vectors $\boldsymbol{E}^{i}$ and the linear functionals on the vectors at $(R, \Theta, Z)$ :

$$
\begin{aligned}
& \boldsymbol{E}^{R} \text { at } \widehat{\boldsymbol{X}}(R, \Theta, Z) \longleftrightarrow d R \text { at }(R, \Theta, Z), \\
& \boldsymbol{E}^{\Theta} \text { at } \widehat{\boldsymbol{X}}(R, \Theta, Z) \longleftrightarrow d \Theta \text { at }(R, \Theta, Z) \text {, } \\
& \boldsymbol{E}^{Z} \text { at } \widehat{\boldsymbol{X}}(R, \Theta, Z) \longleftrightarrow d Z \text { at }(R, \Theta, Z) \text {, }
\end{aligned}
$$

where the linear functionals are defined so that

$$
d \xi^{j}\left[\frac{\partial}{\partial \xi^{i}}\right]=\delta_{i}^{j}, \quad \text { where } \quad \xi^{R}=R, \quad \xi^{\Theta}=\Theta, \quad \xi^{Z}=Z,
$$

where we have used square brackets to indicate that $\partial / \partial \xi^{i}$ is the argument of $d \xi^{j}$. We will need this notation for tangent vectors at $(R, \Theta, Z)$ distinct from those at $\widehat{\boldsymbol{X}}(R, \Theta, Z)$ because we will be considering situations in which there is either no position function $\widehat{\boldsymbol{X}}$ from $\Psi(\mathcal{B})$ or the position function is not globally one-to-one, so that two distinct coordinate points $\left(R_{1}, \Theta_{1}, Z_{1}\right)$ and $\left(R_{2}, \Theta_{2}, Z_{2}\right)$ may be mapped to the same point in $\mathbb{E}^{3}$.

\subsection{Metric Tensor}

A priori, the tangent space at $(R, \Theta, Z) \in \Psi(\mathcal{B})$ is just a real three-dimensional vector space, not an inner-product space. A Riemannian metric tensor is a smooth ${ }^{1}$, symmetric, positivedefinite quadratic form on tangent vectors in $\Psi(\mathcal{B})$. A metric tensor is used to assign sizes to vectors and to compute the analogues of dot-products between vectors. A differentiable manifold equipped with a Riemannian metric tensor is called a Riemannian manifold.

When a position function $\widehat{\boldsymbol{X}}$ is present, the tangent space at $\widehat{\boldsymbol{X}}(R, \Theta, Z)$ is an innerproduct space, and we want the tangent space at $(R, \Theta, Z)$ to "inherit" the inner product. That is, we want an inner product $\langle$,$\rangle such that$

$$
\begin{aligned}
& \left\langle\frac{\partial}{\partial R}, \frac{\partial}{\partial R}\right\rangle=\boldsymbol{E}_{R} \cdot \boldsymbol{E}_{R}=1, \\
& \left\langle\frac{\partial}{\partial \Theta}, \frac{\partial}{\partial \Theta}\right\rangle=\boldsymbol{E}_{\Theta} \cdot \boldsymbol{E}_{\Theta}=R^{2} \\
& \left\langle\frac{\partial}{\partial Z}, \frac{\partial}{\partial Z}\right\rangle=\boldsymbol{E}_{Z} \cdot \boldsymbol{E}_{Z}=1,
\end{aligned}
$$

\footnotetext{
${ }^{1}$ Infinite differentiability is required in Riemannian geometry, but several continuous derivatives are sufficient in much of solid mechanics.
} 
with all other inner products of basis vectors equal to zero. Using the linear functionals in Eq. (12), we can construct a bilinear form that satisfies these relations:

$$
M=d R \otimes d R+R^{2} d \Theta \otimes d \Theta+d Z \otimes d Z
$$

Note the correspondence of $M$ to the tensor

$$
\boldsymbol{M}=\boldsymbol{E}^{R} \otimes \boldsymbol{E}^{R}+R^{2} \boldsymbol{E}^{\Theta} \otimes \boldsymbol{E}^{\Theta}+\boldsymbol{E}^{Z} \otimes \boldsymbol{E}^{Z}
$$

which can be used to define the inner products of $\boldsymbol{E}_{R}, \boldsymbol{E}_{\Theta}$, and $\boldsymbol{E}_{Z}$.

If we treat the functions $E_{i j}=\boldsymbol{E}_{i} \cdot \boldsymbol{E}_{j}$ as functions of $(R, \Theta, Z)$ and ignore their original definition, $M$ in Eq. (15), which is known as a metric tensor, can be expressed as

$$
M=E_{i j} d \xi^{i} \otimes d \xi^{j}
$$

Among other things, the metric tensor defines arclengths of curves. Let $[a, b] \ni s \mapsto$ $\gamma(s)=(R(s), \Theta(s), Z(s))$ be a continuously differentiable curve in $\Psi(\mathcal{B})$. The length of $\gamma$ is computed with the metric tensor as follows:

$$
\int_{a}^{b} \sqrt{E_{i j}(\gamma(s)) \frac{d \xi^{i}}{d s} \frac{d \xi^{j}}{d s}} d s=\int_{a}^{b} \sqrt{\left(R^{\prime}(s)\right)^{2}+\left(R(s) \Theta^{\prime}(s)\right)^{2}+\left(Z^{\prime}(s)\right)^{2}} d s .
$$

By design of $M$, the length in Eq. (18) is the same as the Euclidean length of the Euclidean image $\widehat{\boldsymbol{X}} \circ \gamma$ of the curve. Whenever there is a position function $\widehat{\boldsymbol{X}}$, the metric tensor can be used to convert a triple of coordinate velocities $(d R / d s, d \Theta / d s, d Z / d s)$ into the squared norm of the corresponding Euclidean velocity. First we write the triple of velocities as the tangent vector

$$
\frac{d R}{d S} \frac{\partial}{\partial R}+\frac{d \Theta}{d s} \frac{\partial}{\partial \Theta}+\frac{d Z}{d s} \frac{\partial}{\partial Z}
$$

Then we compute the inner product

$\left\langle\frac{d R}{d S} \frac{\partial}{\partial R}+\frac{d \Theta}{d s} \frac{\partial}{\partial \Theta}+\frac{d Z}{d s} \frac{\partial}{\partial Z}, \frac{d R}{d S} \frac{\partial}{\partial R}+\frac{d \Theta}{d s} \frac{\partial}{\partial \Theta}+\frac{d Z}{d s} \frac{\partial}{\partial Z}\right\rangle=\left(\frac{d R}{d s}\right)^{2}+R^{2}\left(\frac{d \Theta}{d s}\right)^{2}+\left(\frac{d Z}{d s}\right)^{2}$.

Tangent vector at $(R, \Theta, Z) \in \Psi(\mathcal{B})$ corresponds to a tangent vector at $\Psi^{-1}(R, \Theta, Z) \in \mathcal{B}$. Similarly, the metric tensor at $(R, \Theta, Z)$ also corresponds to a symmetric, positive-definite bilinear form on tangent vectors at $\Psi^{-1}(R, \Theta, Z)$. Equipped with this bilinear form, each tangent space at a point in $\mathcal{B}$ becomes an inner-product space, and $\mathcal{B}$ with the abstract metric tensor is an example of a Riemannian manifold.

\subsubsection{Deformation and Change of Metric Tensor}

Consider a deformation $\chi$ in which the deformed configuration has coordinates $(r, \theta, z)$ and corresponding position function

$$
\widehat{\boldsymbol{x}}(r, \theta, z)=r \cos \theta \boldsymbol{i}+r \sin \theta \boldsymbol{j}+z \boldsymbol{k} .
$$




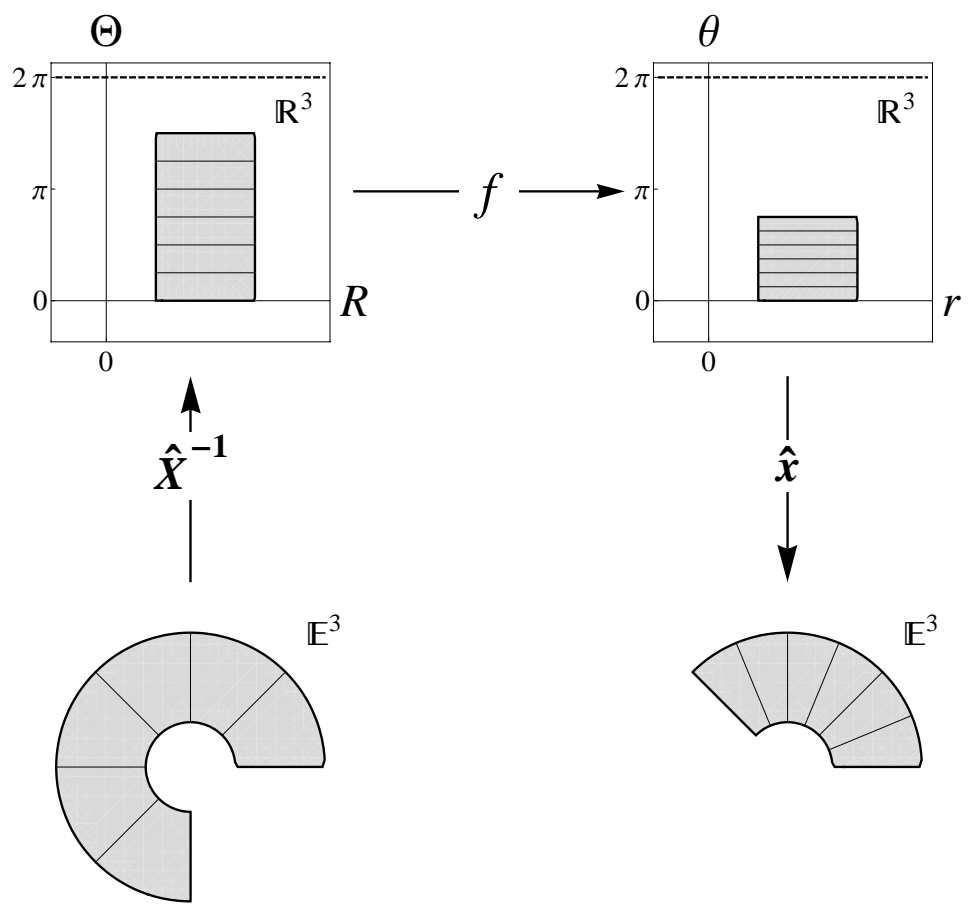

Figure 4: A deformation of a solid body is computed in three steps. $\widehat{\boldsymbol{X}}^{-1}$ maps each Euclidean point of the body to its corresponding coordinate point. $f$ maps reference coordinate points to deformed coordinate points. The position function $\widehat{\boldsymbol{x}}$ maps deformed coordinate points into $\mathbb{E}^{3}$. The full deformation is $\boldsymbol{\chi}=\widehat{\boldsymbol{x}} \circ f \circ \widehat{\boldsymbol{X}}^{-1}$.

Just as the position function $\widehat{\boldsymbol{X}}$ in Eq. (2) induces the metric tensor in Eq. (15) on the space of $(R, \Theta, Z)$-points, the position function $\widehat{\boldsymbol{x}}$ induces a metric tensor on the space of $(r, \theta, z)$-points:

$$
m=d r \otimes d r+r^{2} d \theta \otimes d \theta+d z \otimes d z .
$$

If we use the notation $\left(\xi^{1}, \xi^{2}, \xi^{3}\right)=(R, \Theta, Z)$ for the reference coordinates and $\left(\zeta^{1}, \zeta^{2}, \zeta^{3}\right)=$ $(r, \theta, z)$ for the deformed coordinates, the deformation gradient can be written as

$$
\boldsymbol{F}=\frac{\partial \zeta^{j}}{\partial \xi^{i}} \boldsymbol{e}_{j} \otimes \boldsymbol{E}^{i}
$$

where

$$
\begin{aligned}
\boldsymbol{e}_{r} & =\frac{\partial \widehat{\boldsymbol{x}}}{\partial r}=\cos \theta \boldsymbol{i}+\sin \theta \boldsymbol{j}=\boldsymbol{h}_{1}(\theta) \\
\boldsymbol{e}_{\theta} & =\frac{\partial \widehat{\boldsymbol{x}}}{\partial \theta}=-r \sin \theta \boldsymbol{i}+r \cos \theta \boldsymbol{j}=r \boldsymbol{h}_{2}(\theta), \\
\boldsymbol{e}_{z} & =\frac{\partial \widehat{\boldsymbol{x}}}{\partial z}=\boldsymbol{k} .
\end{aligned}
$$

The deformation gradient maps tangent vectors at $\boldsymbol{X} \in(\widehat{\boldsymbol{X}} \circ \Psi)(\mathcal{B})$ to the corresponding, deformed tangent vectors at $\boldsymbol{x}=\boldsymbol{\chi}(\boldsymbol{X})$. The right Cauchy-Green tensor $\boldsymbol{F}^{T} \cdot \boldsymbol{F}$ associated 
with $\boldsymbol{F}$ provides a quadratic form akin to the one in Eq. (16):

$$
\begin{aligned}
\boldsymbol{F}^{T} \cdot \boldsymbol{F} & =\left(\frac{\partial \zeta^{j}}{\partial \xi^{i}} \boldsymbol{E}^{i} \otimes \boldsymbol{e}_{j}\right) \cdot\left(\frac{\partial \zeta^{\ell}}{\partial \xi^{k}} \boldsymbol{e}_{\ell} \otimes \boldsymbol{E}^{k}\right) \\
& =e_{j \ell} \frac{\partial \zeta^{j}}{\partial \xi^{i}} \frac{\partial \zeta^{\ell}}{\partial \xi^{k}} \boldsymbol{E}^{i} \otimes \boldsymbol{E}^{k}
\end{aligned}
$$

where $e_{j \ell}=\boldsymbol{e}_{j} \cdot \boldsymbol{e}_{\ell} \cdot \boldsymbol{F}^{T} \cdot \boldsymbol{F}$ allows the computation of deformed arclengths while using the reference coordinates $(R, \Theta, Z)$. For example, if $[a, b] \ni s \mapsto \gamma(s)=(R(s), \Theta(s), Z(s))$ is a continuously differentiable curve in $\Psi(\mathcal{B})$, then the velocity of its Euclidean image is

$$
\boldsymbol{v}=\frac{d R}{d s} \boldsymbol{E}_{R}+\frac{d \Theta}{d s} \boldsymbol{E}_{\Theta}+\frac{d Z}{d s} \boldsymbol{E}_{Z}
$$

and its arclength after deformation is

$$
\begin{aligned}
\int_{a}^{b}\|\boldsymbol{F} \cdot \boldsymbol{v}\| d s & =\int_{a}^{b} \sqrt{\boldsymbol{v} \cdot \boldsymbol{F}^{T} \cdot \boldsymbol{F} \cdot \boldsymbol{v}} d s \\
& =\int_{a}^{b} \sqrt{e_{j \ell} \frac{\partial \zeta^{j}}{\partial \xi^{i}} \frac{\partial \zeta^{\ell}}{\partial \xi^{k}} \frac{d \xi^{i}}{d s} \frac{d \xi^{k}}{d s} d s}
\end{aligned}
$$

The metric tensor on $\Psi(\mathcal{B})$ corresponding to the right Cauchy-Green tensor is

$$
e_{j \ell} \frac{\partial \zeta^{j}}{\partial \xi^{i}} \frac{\partial \zeta^{\ell}}{\partial \xi^{k}} d \xi^{i} \otimes d \xi^{k},
$$

which is used as a quadratic form on velocity vectors in $\Psi(\mathcal{B})$ of the form

$$
\mathbf{v}=\frac{d \xi^{i}}{d s} \frac{\partial}{\partial \xi^{i}}=\frac{d R}{d s} \frac{\partial}{\partial R}+\frac{d \Theta}{d s} \frac{\partial}{\partial \Theta}+\frac{d Z}{d s} \frac{\partial}{\partial Z}
$$

If $\mathbf{v}$ is the velocity vector of the curve $s \mapsto \gamma(s)$ in $\Psi(\mathcal{B})$, then the arclength assigned by the metric tensor in Eq. (30) is

$$
\begin{aligned}
\int_{a}^{b} \sqrt{e_{j \ell} \frac{\partial \zeta^{j}}{\partial \xi^{i}} \frac{\partial \zeta^{\ell}}{\partial \xi^{k}} d \xi^{i}[\mathbf{v}] \otimes d \xi^{k}[\mathbf{v}] d s} & =\int_{a}^{b} \sqrt{e_{j \ell} \frac{\partial \zeta^{j}}{\partial \xi^{i}} \frac{\partial \zeta^{\ell}}{\partial \xi^{k}} d \xi^{i}\left[\frac{d \xi^{m}}{d s} \frac{\partial}{\partial \xi^{m}}\right] \otimes d \xi^{k}\left[\frac{d \xi^{n}}{d s} \frac{\partial}{\partial \xi^{n}}\right]} d s \\
& =\int_{a}^{b} \sqrt{e_{j \ell} \frac{\partial \zeta^{j}}{\partial \xi^{i}} \frac{\partial \zeta^{\ell}}{\partial \xi^{k}} \frac{d \xi^{m}}{d s} \delta_{m}^{i} \frac{d \xi^{n}}{d s} \delta_{n}^{k}} d s \\
& =\int_{a}^{b} \sqrt{e_{j \ell} \frac{\partial \zeta^{j}}{\partial \xi^{m}} \frac{\partial \zeta^{\ell}}{\partial \xi^{n}} \frac{d \xi^{m}}{d s} \frac{d \xi^{n}}{d s}} d s
\end{aligned}
$$

The metric tensor in Eq. (30) is called the pull-back of the metric tensor $m$ in Eq. (17), under the map $f(R, \Theta, Z)=(r, \theta, z)$. 


\subsection{Incompatible Growth}

Each fixed two-point tensor with positive determinant can be the value of a deformation gradient at a point, but not every two-point tensor field with positive determinant is equal to a deformation gradient. A continuously differentiable two-point tensor field $\boldsymbol{G}$ with positive determinant will be called an incompatible growth field if it is not equal to a deformation gradient.

Even if $\boldsymbol{G}$ is an incompatible growth field, it can be used to create a new metric tensor in the same fashion that a deformation gradient can. Let

$$
\boldsymbol{G}=G_{i}^{j} \boldsymbol{e}_{i} \otimes \boldsymbol{E}^{j}
$$

Then the analogue of the right Cauchy-Green tensor is

$$
\boldsymbol{G}^{T} \cdot \boldsymbol{G}=e_{j \ell} G_{i}^{j} G_{k}^{\ell} \boldsymbol{E}^{i} \otimes \boldsymbol{E}^{k}
$$

This is a symmetric, positive-definite quadratic form on tangent vectors at $\boldsymbol{X} \in(\widehat{\boldsymbol{X}} \circ \Psi)(\mathcal{B})$, and it corresponds to the metric tensor

$$
e_{j \ell} G_{i}^{j} G_{k}^{\ell} d \xi^{i} \otimes d \xi^{k}
$$

on $\Psi(\mathcal{B})$.

Before we can demonstrate the ways in which $\boldsymbol{G}$ can fail to be a deformation gradient, we must delve a little deeper into differential geometry.

\subsection{Immersibility and Embeddability}

\subsubsection{Immersions, Embeddings, and Isometry}

An immersion of a set $\Omega \subset \mathbb{R}^{3}$ of coordinate points $\left(\xi^{1}, \xi^{2}, \xi^{3}\right)$ is a differentiable function $\widehat{\boldsymbol{Y}}: \Omega \rightarrow \mathbb{E}^{3}$ such that the determinant of the gradient of $\widehat{\boldsymbol{Y}}$ has positive determinant throughout $\Omega$ :

$$
\operatorname{det}\left(\frac{\partial \widehat{\boldsymbol{Y}}}{\partial \xi^{1}} \frac{\partial \widehat{\boldsymbol{Y}}}{\partial \xi^{2}} \frac{\partial \widehat{\boldsymbol{Y}}}{\partial \xi^{3}}\right)>0 .
$$

This ensures that no volumes in $\Omega$ are collapsed to areas in $\mathbb{E}^{3}$, and that no volumes undergo a reversal of orientation. If $\Omega$ is equipped with a metric tensor $E_{i j} d \xi^{i} \otimes d \xi^{j}$, an immersion $\widehat{\boldsymbol{Y}}$ is called isometric if

$$
\frac{\partial \widehat{\boldsymbol{Y}}}{\partial \xi^{i}} \cdot \frac{\partial \widehat{\boldsymbol{Y}}}{\partial \xi^{j}}=E_{i j}, \quad i, j=1,2,3, \text { in } \Omega
$$

An immersion is locally invertible, but it need not be globally so. An immersion $\widehat{\boldsymbol{Y}}$ of $\Omega$ is called an embedding if it invertible throughout $\Omega$. An embedding is called isometric if it satisfies Eq. (37). 


\subsubsection{Riemann-Christoffel Curvature Tensor}

Not every Riemannian metric has an isometric immersion into $\mathbb{E}^{3}$. There is a test for the existence of such a map from coordinates to $\mathbb{E}^{3}$. The test involves the Riemann-Christoffel curvature tensor, whose computation we now show.

If $E_{i j} d \xi^{i} \otimes d \xi^{j}$ is a Riemannian metric, then the Christoffel symbols of the first kind are defined by

$$
\Gamma_{i j k}=\frac{1}{2}\left(\frac{\partial E_{i k}}{\partial \xi^{j}}+\frac{\partial E_{j k}}{\partial \xi^{i}}-\frac{\partial E_{i j}}{\partial \xi^{k}}\right),
$$

and the Christoffel symbols of the second kind are defined by

$$
\Gamma_{i j}^{\ell}=E^{\ell k} \Gamma_{i j k},
$$

where $\left[E^{i j}\right]=\left[E_{i j}\right]^{-1}$.

In classical tensor analysis, the Christoffel symbols of the second kind are defined as the coefficients used to express the rates of change of coordinate tangent vectors:

$$
\frac{\partial \boldsymbol{E}_{j}}{\partial \xi^{i}}=\Gamma_{i j}^{\ell} \boldsymbol{E}_{\ell}
$$

and the Christoffel symbols of the first kind are defined by "lowering" the raised index in $\Gamma_{i j}^{\ell}$ :

$$
\Gamma_{i j k}=E_{k \ell} \Gamma_{i j}^{\ell} .
$$

The Riemann-Christoffel curvature tensor $\mathcal{R}$ is a four-indexed tensor whose components satisfy

$$
\mathcal{R}_{\ell i j k}=\frac{\partial \Gamma_{i k \ell}}{\partial \xi^{j}}-\frac{\partial \Gamma_{i j \ell}}{\partial \xi^{k}}+\Gamma_{i j}^{m} \Gamma_{k \ell m}-\Gamma_{i k}^{m} \Gamma_{j \ell m} .
$$

The test is embodied in the three-dimensional case of the "Fundamental Theorem of Riemannian Geometry", as described in Theorem 1.6-1 in [6], which we paraphrase:

Theorem 2.1 Let $\Omega$ be an open, simply-connected set in $\mathbb{R}^{3}$ and let $\boldsymbol{C}=\left[E_{i j}\right]$ be a $C^{2}$ symmetric positive-definite matrix-valued function on $\Omega$ that satisfies

$$
\mathcal{R} \equiv \boldsymbol{O} \text { in } \Omega
$$

where $\mathcal{R}_{\ell i j k}$ is defined by Eq. (39). Then there exists a $C^{3}$ immersion $\widehat{\boldsymbol{Y}}$ of $\Omega$ such that

$$
E_{i j}=\frac{\partial \widehat{\boldsymbol{Y}}}{\partial \xi^{i}} \cdot \frac{\partial \widehat{\boldsymbol{Y}}}{\partial \xi^{j}} \quad \text { in } \Omega .
$$

For a deeper exploration, see [6] or [11].

We will consider simple cases in which isometric embeddability fails. 


\subsection{Example: Immersion without Embedding}

We construct an example by considering two manifolds with cylindrical coordinate systems, metric tensors, and position functions isometric with the given metric tensors:

$\begin{array}{ll}\text { manifold } & \mathcal{B} \\ \text { coordinate system } & \Psi=(R, \Theta, Z) \\ \text { metric tensor } & M=d R \otimes d R+R^{2} d \Theta \otimes d \Theta+d Z \otimes d Z \\ & \boldsymbol{M}=\boldsymbol{E}^{R} \otimes \boldsymbol{E}^{R}+R^{2} \boldsymbol{E}^{\Theta} \otimes \boldsymbol{E}^{\Theta}+\boldsymbol{E}^{Z} \otimes \boldsymbol{E}^{Z} \\ \text { isometric position function } & \widehat{\boldsymbol{X}}(R, \Theta, Z)=R \cos (\Theta) \boldsymbol{i}+R \sin (\Theta) \boldsymbol{j}+Z \boldsymbol{k} \\ & \mathcal{M} \\ \text { manifold } & \psi=(r, \theta, z) \\ \text { coordinate system } & m=d r \otimes d r+r^{2} d \theta \otimes d \theta+d z \otimes d z \\ \text { metric tensor } & \boldsymbol{m}=\boldsymbol{e}^{r} \otimes \boldsymbol{e}^{r}+r^{2} \boldsymbol{e}^{\theta} \otimes \boldsymbol{e}^{\theta}+\boldsymbol{e}^{z} \otimes \boldsymbol{e}^{z} \\ & \widehat{\boldsymbol{x}}(r, \theta, z)=r \cos (\theta) \boldsymbol{i}+r \sin (\theta) \boldsymbol{j}+z \boldsymbol{k}\end{array}$

In each case we have also included the "Euclidean image" of the metric tensor under the isometric position functions, where

$$
\begin{aligned}
\boldsymbol{e}^{r} & =\cos (\theta) \boldsymbol{i}+\sin (\theta) \boldsymbol{j} \\
\boldsymbol{e}^{\theta} & =-r^{-1} \sin (\theta) \boldsymbol{i}+r^{-1} \cos (\theta) \boldsymbol{j} \\
\boldsymbol{e}^{z} & =\boldsymbol{k} .
\end{aligned}
$$

We consider a two-point tensor field of the form

$$
\boldsymbol{G}=\gamma_{1} \boldsymbol{e}_{r} \otimes \boldsymbol{E}^{R}+\frac{\gamma_{2}}{\gamma_{2}} \boldsymbol{e}_{\theta} \otimes \boldsymbol{E}^{\Theta}+\boldsymbol{e}_{z} \otimes \boldsymbol{E}^{Z}
$$

where $\gamma_{1}$ and $\gamma_{2}$ are positive constants. If this were a deformation gradient, the reference coordinates-to-deformed coordinates part of the deformation would have the form

$$
r=\gamma_{1} R, \quad \theta=\frac{\gamma_{2}}{\gamma_{1}} \Theta, \quad z=Z
$$

The analogue of the right Cauchy-Green tensor is

$$
\begin{aligned}
\boldsymbol{G}^{T} \cdot \boldsymbol{G} & =\left(\gamma_{1} \boldsymbol{E}^{R} \otimes \boldsymbol{e}_{r}+\frac{\gamma_{2}}{\gamma_{2}} \boldsymbol{E}^{\Theta} \otimes \boldsymbol{e}_{\theta}+\boldsymbol{E}^{Z} \otimes \boldsymbol{e}_{z}\right) \cdot\left(\gamma_{1} \boldsymbol{e}_{r} \otimes \boldsymbol{E}^{R}+\frac{\gamma_{2}}{\gamma_{2}} \boldsymbol{e}_{\theta} \otimes \boldsymbol{E}^{\Theta}+\boldsymbol{e}_{z} \otimes \boldsymbol{E}^{Z}\right) \\
& =\gamma_{1}^{2} e_{r r} \boldsymbol{E}^{R} \otimes \boldsymbol{E}^{R}+\left(\frac{\gamma_{2}}{\gamma_{1}}\right)^{2} e_{\theta \theta} \boldsymbol{E}^{\Theta} \otimes \boldsymbol{E}^{\Theta}+e_{z z} \boldsymbol{E}^{Z} \otimes \boldsymbol{E}^{Z} \\
& =\gamma_{1}^{2} \boldsymbol{E}^{R} \otimes \boldsymbol{E}^{R}+\left(\frac{\gamma_{2}}{\gamma_{1}}\right)^{2} r^{2} \boldsymbol{E}^{\Theta} \otimes \boldsymbol{E}^{\Theta}+\boldsymbol{E}^{Z} \otimes \boldsymbol{E}^{Z} \\
& =\gamma_{1}^{2} \boldsymbol{E}^{R} \otimes \boldsymbol{E}^{R}+\gamma_{2}^{2} R^{2} \boldsymbol{E}^{\Theta} \otimes \boldsymbol{E}^{\Theta}+\boldsymbol{E}^{Z} \otimes \boldsymbol{E}^{Z}
\end{aligned}
$$

This corresponds to the metric tensor

$$
\gamma_{1}^{2} d R \otimes d R+\gamma_{2}^{2} R^{2} d \Theta \otimes d \Theta+d Z \otimes d Z
$$


on $\Psi(\mathcal{B})$. Applying Eqs. (38), (39), and (42) to the coefficients of the this metric yields an identically zero Riemann-Christoffel curvature tensor. Theorem 2.1 ensures the existence of an isometric immersion of any simply-connected subset of $\Psi(\mathcal{B})$.

Consider the maps $\widehat{\boldsymbol{x}} \circ f: \Psi(\mathcal{B}) \rightarrow \mathbb{E}^{3}$ shown in Figures 5 and 6 :

$$
\begin{aligned}
(\widehat{\boldsymbol{x}} \circ f)(R, \Theta, Z) & =\widehat{\boldsymbol{x}}\left(\gamma_{1} R, \frac{\gamma_{2}}{\gamma_{1}} \Theta, Z\right) \\
& =\gamma_{1} R \cos \left(\frac{\gamma_{2}}{\gamma_{1}} \Theta\right) \boldsymbol{i}+\gamma_{1} R \sin \left(\frac{\gamma_{2}}{\gamma_{1}} \Theta\right) \boldsymbol{j}+Z \boldsymbol{k} .
\end{aligned}
$$

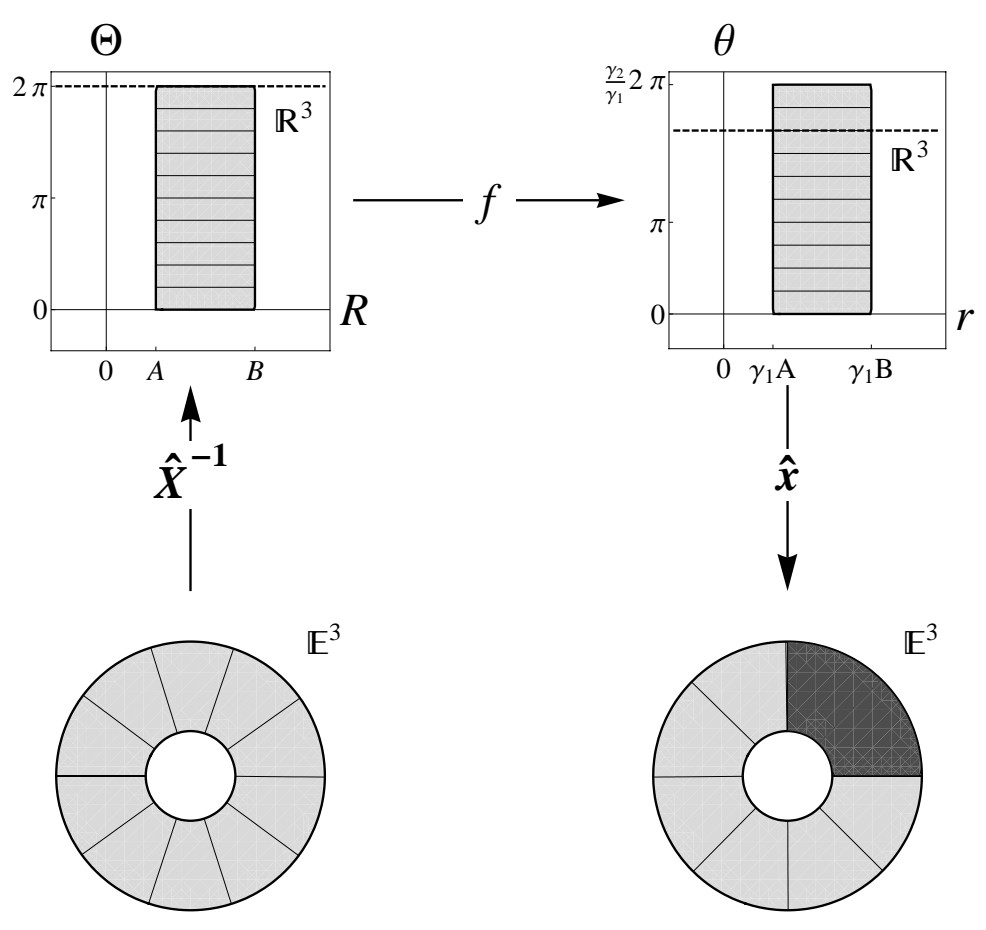

Figure 5: If $\gamma_{2}>\gamma_{1}$, then $\widehat{\boldsymbol{x}} \circ f: \Psi(\mathcal{B}) \rightarrow \mathbb{E}^{3}$ double-covers part of $\mathbb{E}^{3}$.

The coordinate basis vectors with respect to $(R, \Theta, Z)$ are

$$
\begin{aligned}
& \frac{\partial(\widehat{\boldsymbol{x}} \circ f)}{\partial R}=\gamma_{1} \cos \left(\frac{\gamma_{2}}{\gamma_{1}} \Theta\right) \boldsymbol{i}+\gamma_{1} \sin \left(\frac{\gamma_{2}}{\gamma_{1}} \Theta\right) \boldsymbol{j} \\
& \frac{\partial(\widehat{\boldsymbol{x}} \circ f)}{\partial \Theta}=-\gamma_{2} R \sin \left(\frac{\gamma_{2}}{\gamma_{1}} \Theta\right) \boldsymbol{i}+\gamma_{2} R \cos \left(\frac{\gamma_{2}}{\gamma_{1}} \Theta\right) \boldsymbol{j} \\
& \frac{\partial(\widehat{\boldsymbol{x}} \circ f)}{\partial Z}=\boldsymbol{k},
\end{aligned}
$$

whose dot products form a symmetric positive-definite matrix:

$$
\left(\begin{array}{ccc}
\gamma_{1}^{2} & 0 & 0 \\
0 & \gamma_{2} R^{2} & 0 \\
0 & 0 & 1
\end{array}\right)
$$




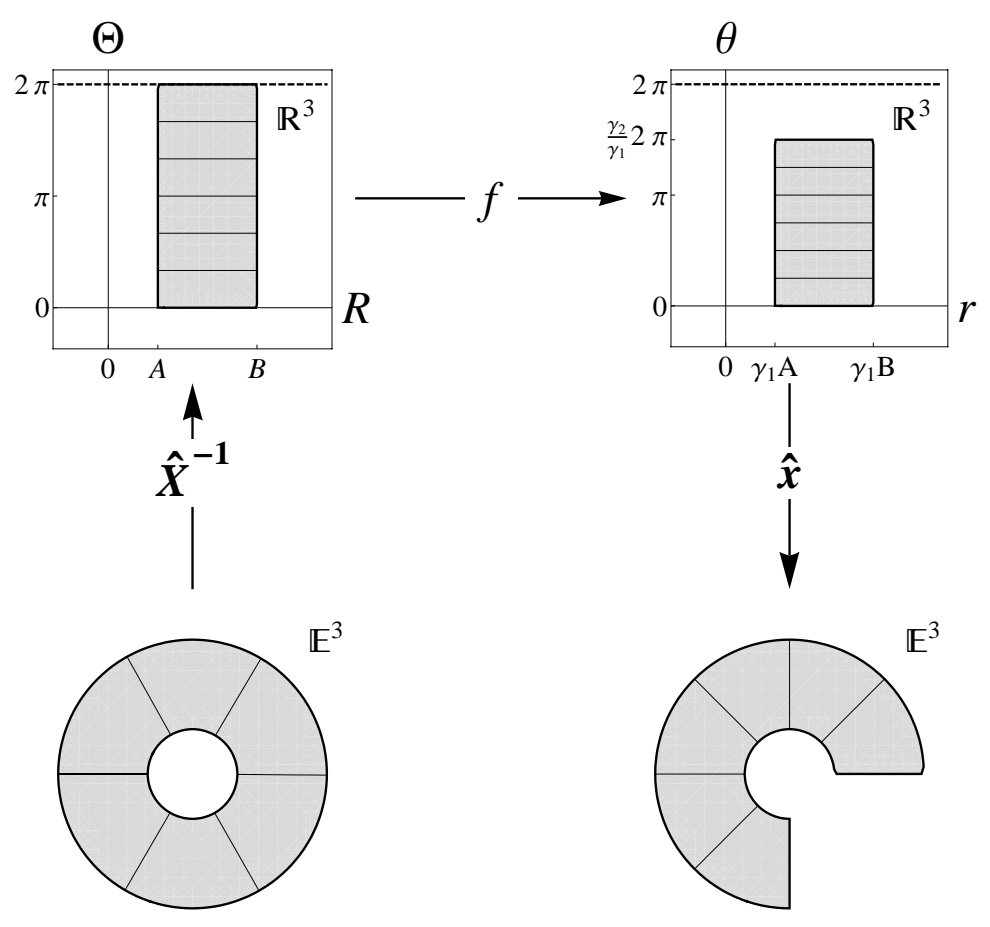

Figure 6: If $\gamma_{2}<\gamma_{1}$, then $\widehat{\boldsymbol{x}} \circ f$ does not map $\Psi(\mathcal{B})$ to a complete annulus in $\mathbb{E}^{3}$.

Thus $\widehat{\boldsymbol{x}} \circ f$ maps $\Psi(\mathcal{B})$ into $\mathbb{E}^{3}$ in an isometric immersion of $\Psi(\mathcal{B})$ with the metric in Eq. (47). But Figures 5 and 6 reveal that if $\gamma_{1} \neq \gamma_{2}$, then the image of $\Psi(\mathcal{B})$ under $\widehat{\boldsymbol{x}} \circ f$ is not a proper annulus.

A theorem on the "rigidity" of the class of isometric immersions shows that the problems found in $\widehat{\boldsymbol{x}} \circ f$ will be found in any isometric immersion of $\Psi(\mathcal{B})$ with the metric tensor in Eq. (47):

Theorem 2.2 Let $\Omega$ be an open, connected subset of $\mathbb{R}^{3}$, and let $\widehat{\boldsymbol{Y}}$ and $\widehat{\boldsymbol{Z}}$ be two $C^{1}$ immersions such that their associated metric tensors satisfy

$$
\frac{\partial \widehat{\boldsymbol{Y}}}{\partial \xi^{i}} \cdot \frac{\partial \widehat{\boldsymbol{Y}}}{\partial \xi^{j}}=\frac{\partial \widehat{\boldsymbol{Z}}}{\partial \xi^{i}} \cdot \frac{\partial \widehat{\boldsymbol{Z}}}{\partial \xi^{j}} \quad \text { in } \Omega .
$$

Then there exist a constant vector $\boldsymbol{c}$ and a constant orthogonal matrix $\boldsymbol{Q}$ such that

$$
\widehat{\boldsymbol{Z}}\left(\xi^{1}, \xi^{2}, \xi^{3}\right)=\boldsymbol{Q} \cdot \widehat{\boldsymbol{Y}}\left(\xi^{1}, \xi^{2}, \xi^{3}\right)+\boldsymbol{c} \text { for each }\left(\xi^{1}, \xi^{2}, \xi^{3}\right) \in \Omega .
$$

See Theorem $1.7-1$ in $[6]$.

No constant rotation and translation of $\widehat{\boldsymbol{x}} \circ f$ will solve the problems displayed in Figures 5 and 6. The annulus cannot be embedded in $\mathbb{E}^{3}$ in a fashion that agrees with the metric in Eq. (47). The map $\boldsymbol{\chi}=\widehat{\boldsymbol{x}} \circ f \circ \widehat{\boldsymbol{X}}^{-1}$ described above is a local deformation on subsets of the annulus, but it is not a global deformation. Hence $\boldsymbol{G}$ is not equal to a global deformation gradient; it is an incompatible growth field. 


\subsection{Lack of Immersion}

Consider next a growth field of the form

$$
\boldsymbol{G}=\gamma_{1} \boldsymbol{e}_{r} \otimes \boldsymbol{E}^{R}+\frac{\gamma_{2} R}{\int_{0}^{R} \gamma_{1}(s) d s} \boldsymbol{e}_{\theta} \otimes \boldsymbol{E}^{\Theta}+\boldsymbol{e}_{z} \otimes \boldsymbol{E}^{Z},
$$

where $\gamma_{1}$ and $\gamma_{2}$ are positive, continuously differentiable functions of $R$, and

$$
r(R)=\int_{0}^{R} \gamma_{1}(s) d s, \quad \theta(R, \Theta)=\frac{\gamma_{2}(R) R}{\int_{0}^{R} \gamma_{1}(s) d s} \Theta, \quad z=Z .
$$

The analogue of the right Cauchy-Green tensor is

$$
\begin{aligned}
\boldsymbol{G}^{T} \cdot \boldsymbol{G} & =\gamma_{1}^{2} e_{r r} \boldsymbol{E}^{R} \otimes \boldsymbol{E}^{R}+\left(\frac{\gamma_{2} R}{\int_{0}^{R} \gamma_{1}(s) d s}\right)^{2} e_{\theta \theta} \boldsymbol{E}^{\Theta} \otimes \boldsymbol{E}^{\Theta}+e_{z z} \boldsymbol{E}^{Z} \otimes \boldsymbol{E}^{Z} \\
& =\gamma_{1}^{2} \boldsymbol{E}^{R} \otimes \boldsymbol{E}^{R}+\left(\frac{\gamma_{2} R}{\int_{0}^{R} \gamma_{1}(s) d s}\right)^{2} r^{2} \boldsymbol{E}^{\Theta} \otimes \boldsymbol{E}^{\Theta}+\boldsymbol{E}^{Z} \otimes \boldsymbol{E}^{Z} \\
& =\gamma_{1}^{2} \boldsymbol{E}^{R} \otimes \boldsymbol{E}^{R}+\gamma_{2}^{2} R^{2} \boldsymbol{E}^{\Theta} \otimes \boldsymbol{E}^{\Theta}+\boldsymbol{E}^{Z} \otimes \boldsymbol{E}^{Z}
\end{aligned}
$$

which corresponds to the metric tensor

$$
\gamma_{1}^{2} d R \otimes d R+\gamma_{2}^{2} R^{2} d \Theta \otimes d \Theta+d Z \otimes d Z
$$

on $\Psi(\mathcal{B})$. Applying Eqs. (38), (39), and (42) to the coefficients of the metric in Eq. (54) yields a Riemann-Christoffel curvature tensor with four entries that may be nonzero:

$$
\mathcal{R}_{R \Theta R \Theta}=-\mathcal{R}_{R \Theta \Theta R}=\mathcal{R}_{\Theta R \Theta R}=-\mathcal{R}_{\Theta R R \Theta}=\frac{R \gamma_{2}}{\gamma_{1}}\left(\gamma_{2} \gamma_{1}^{\prime}+R \gamma_{1}^{\prime} \gamma_{2}^{\prime}-\gamma_{1}\left(2 \gamma_{2}^{\prime}+R \gamma_{2}^{\prime \prime}\right)\right)
$$

Unless

$$
\frac{\gamma_{2}+R \gamma_{2}^{\prime}}{\gamma_{1}}=\text { constant }
$$

the two-point tensor field $\boldsymbol{G}$ in Eq. (51) is an incompatible growth field.

\subsection{Non-Metric Complications}

Note that computing the Riemann-Christoffel curvature tensor tests properties of $\boldsymbol{G}^{T} \cdot \boldsymbol{G}$ and not of $\boldsymbol{G}$ alone. We provide here an example of a two-point tensor field with positive determinant and identically zero associated Riemann-Christoffel curvature that is not a deformation gradient, even locally.

Let

$$
\boldsymbol{G}=\left(\cos \phi \boldsymbol{e}_{r}-\sin \phi \boldsymbol{e}_{z}\right) \otimes \boldsymbol{E}^{R}+\left(-\sin \phi \boldsymbol{e}_{r}+\sin \phi \boldsymbol{e}_{z}\right) \otimes \boldsymbol{E}^{Z}+\boldsymbol{e}_{\theta} \otimes \boldsymbol{E}^{\Theta}
$$


with $r=R, \theta=\Theta$, and $z=Z$. $\phi$ is an $R$-dependent angle. This corresponds to a rotation of the vectors $\boldsymbol{e}_{r}$ and $\boldsymbol{e}_{z}$ in the $\theta=$ constant plane. The analogue of the right Cauchy-Green tensor is

$$
\begin{aligned}
\boldsymbol{G}^{T} \cdot \boldsymbol{G} & =\left(e_{r r} \cos ^{2} \phi+e_{z z} \sin ^{2} \phi\right) \boldsymbol{E}^{R} \otimes \boldsymbol{E}^{R}+e_{\theta \theta} \boldsymbol{E}^{\Theta} \otimes \boldsymbol{E}^{\Theta}+\left(e_{r r} \sin ^{2} \phi+e_{z z} \cos ^{2} \phi\right) \boldsymbol{E}^{Z} \otimes \boldsymbol{E}^{Z} \\
& =\boldsymbol{E}^{R} \otimes \boldsymbol{E}^{R}+R^{2} \boldsymbol{E}^{\Theta} \otimes \boldsymbol{E}^{\Theta}+\boldsymbol{E}^{Z} \otimes \boldsymbol{E}^{Z}
\end{aligned}
$$

which is the same as the Euclidean metric tensor induced by the position function $\widehat{\boldsymbol{X}}$ from cylindrical coordinates to $\mathbb{E}^{3}$. The associated Riemann-Christoffel curvature tensor is identically zero. This tells us that $\boldsymbol{G}^{T} \cdot \boldsymbol{G}$ is equal to the right Cauchy-Green tensor for some isometric immersion. It does not imply, however, that $\boldsymbol{G}$ is itself the gradient of an iometric immersion.

Consider the form of a deformation gradient using cylindrical coordinates $(R, \Theta, Z)$ and $(r, \theta, Z)$ :

$$
\begin{aligned}
& \boldsymbol{F}=\boldsymbol{e}_{r} \otimes \underbrace{\left(\frac{\partial r}{\partial R} \boldsymbol{E}^{R}+\frac{\partial r}{\partial \Theta} \boldsymbol{E}^{\Theta}+\frac{\partial r}{\partial Z} \boldsymbol{E}^{Z}\right)}_{\mathrm{GRAD} r}+\boldsymbol{e}_{\theta} \otimes \underbrace{\left(\frac{\partial \theta}{\partial R} \boldsymbol{E}^{R}+\frac{\partial \theta}{\partial \Theta} \boldsymbol{E}^{\Theta}+\frac{\partial \theta}{\partial Z} \boldsymbol{E}^{Z}\right)}_{\mathrm{GRAD} \theta} \\
&+\boldsymbol{e}_{z} \otimes \underbrace{\left(\frac{\partial z}{\partial R} \boldsymbol{E}^{R}+\frac{\partial z}{\partial \Theta} \boldsymbol{E}^{\Theta}+\frac{\partial z}{\partial Z} \boldsymbol{E}^{Z}\right)}_{\mathrm{GRAD} z} .
\end{aligned}
$$

If $\boldsymbol{G}$ in Eq. (57) is a deformation gradient, then

$$
\text { GRAD } r=\cos \phi \boldsymbol{E}^{R}-\sin \phi \boldsymbol{E}^{Z}
$$

and the vector field on the right-hand side should have identically zero curl. However,

$$
\operatorname{CURL}\left(\cos \phi \boldsymbol{E}^{R}-\sin \phi \boldsymbol{E}^{Z}\right)=R \phi^{\prime} \cos \phi \boldsymbol{E}^{\Theta},
$$

so for non-constant $\phi$ the two-point tensor field $\boldsymbol{G}$ in Eq. (57) is not an isometric immersion.

All information about orientation is absent from $\boldsymbol{G}^{T} \cdot \boldsymbol{G}$. $\boldsymbol{G}$ fails to be even a local deformation gradient because it describes a re-orientation of material fibers without any bending, stretching, or compression of the body. If the material fibers were re-oriented in this fashion in a deformation, the result of this microscopic re-orientation would be a macroscopic change in the shape of the body.

Considering incompatible growth such as that in Eq. (57) requires more advanced differential geometry, including the theory of linear connections. Incompatible growth of this kind will not be considered here.

\section{Deformation and Hyperelasticity}

\subsection{The Multiplicative Decomposition}

We imagine incompatible growth to be non-elastic, i.e. it generates no elastic strain energy. A Riemannian manifold that undergoes incompatible growth has intrinsic arclengths that 
preclude it from being isometrically immersed in $\mathbb{E}^{3}$. Since a solid body must be embedded isometrically in $\mathbb{E}^{3}$, the transformation of an incompatibly grown body is incomplete without some distortion that changes the "grown" metric tensor to one that has allows isometric immersion.

We assume that the body has an elastic response to the incompatible growth, and we assume that the full deformation gradient $\boldsymbol{F}$ can be decomposed as in [14] and related works:

$$
\boldsymbol{F}=\boldsymbol{A} \cdot \boldsymbol{G},
$$

where $\boldsymbol{A}$ is a two-point tensor field that describes the distortion caused by the body's elastic response to the incompatible growth field $\boldsymbol{G}$. A rough description is that the incompatible growth, described by $\boldsymbol{G}$, transforms infinitesimal neighborhoods in such a way that they cannot fit together into $\mathbb{E}^{3}$, and the elastic response, described by $\boldsymbol{A}$, transforms these neighborhoods so that they again form a solid body in $\mathbb{E}^{3}$, without overlaps or voids.

\subsection{Constitutive Relation of an Elastic Body with Growth}

\subsubsection{Traditional Hyperelasticity}

A growthless hyperelastic body has a strain-energy density $W(\boldsymbol{F})$ (a function of the CauchyGreen tensor $\boldsymbol{F} \cdot \boldsymbol{F}^{T}$ in an isotropic material) with units

$$
[W(\boldsymbol{F})]=\frac{\text { elastic energy }}{\text { reference volume }} .
$$

The nominal stress tensor, which is the transpose of the first Piola-Kirchhoff stress tensor, is

so the units of $\boldsymbol{S}$ are

$$
\boldsymbol{S}=\frac{\partial}{\partial \boldsymbol{F}} W(\boldsymbol{F})
$$

$$
[\boldsymbol{S}]=\frac{[W(\boldsymbol{F})]}{[\boldsymbol{F}]}=\frac{\text { elastic energy / reference volume }}{\text { deformed length / reference length }}=\frac{\text { elastic energy / deformed length }}{\text { reference area }} .
$$

If we recognize elastic energy per final length as elastic force in the deformed configuration, then the units of $\boldsymbol{S}$ reflect $\boldsymbol{S}$ 's rôle as a linear map converting area in the reference configuration into stress in the deformed configuration.

The Cauchy stress tensor $\boldsymbol{T}$ is related to $\boldsymbol{S}$ by

$$
\boldsymbol{T}=(\operatorname{det} \boldsymbol{F})^{-1} \boldsymbol{F} \cdot \boldsymbol{S}
$$

so the units of $\boldsymbol{T}$ are

$$
\begin{aligned}
{[\boldsymbol{T}] } & =[\operatorname{det} \boldsymbol{F}]^{-1}[\boldsymbol{F}][\boldsymbol{S}] \\
& =\left(\frac{\text { deformed volume }}{\text { reference volume }}\right)^{-1} \frac{\text { deformed length }}{\text { reference length }} \cdot \frac{\text { elastic energy / deformed length }}{\text { reference area }} \\
& =\frac{\text { elastic energy } / \text { deformed length }}{\text { deformed area }}
\end{aligned}
$$

These units reflect $\boldsymbol{T}$ 's rôle as a linear map converting area in the deformed configuration to force in the deformed configuration. 


\subsubsection{Hyperelasticity with Growth}

In a body with growth included via the multiplicative decomposition in Eq. (62), we distinguish three states of the body. The pre-growth state is the traditional reference configuration; the post-growth, pre-elastic response state (described by a non-embeddable Riemannian manifold) is called intermediate; and the final (Euclidean) configuration is found after the elastic response to the growth. The lengths, areas, and volumes attached to these three states will be considered distinct.

In this model, the elastic strain-energy density's argument is $\boldsymbol{A}$, the sole (first-order) descriptor of the elastic response. Just as $\boldsymbol{F}$ maps from a tangent space in the reference configuration to a tangent space in the deformed configuration in traditional hyperelasticity, $\boldsymbol{A}$ maps from a tangent space in the intermediate configuration to a tangent space in the final configuration. Considering the units of $W(\boldsymbol{F})$ in Eq. (63), we conclude that the units of $W(\boldsymbol{A})$ are

$$
[W(\boldsymbol{A})]=\frac{\text { elastic energy }}{\text { intermediate volume }} .
$$

The analogue of the nominal stress has units

$$
\left[\frac{\partial}{\partial \boldsymbol{A}} W(\boldsymbol{A})\right]=\frac{\text { elastic energy / intermediate volume }}{\text { final length / intermediate length }}=\frac{\text { elastic energy / final length }}{\text { intermediate area }},
$$

while the analogue of the Cauchy stress has units

$$
\begin{aligned}
& {\left[(\operatorname{det} \boldsymbol{A})^{-1} \boldsymbol{A} \cdot \frac{\partial}{\partial \boldsymbol{A}} W(\boldsymbol{A})\right] } \\
= & \left(\frac{\text { final volume }}{\text { intermediate volume }}\right)^{-1} \cdot \frac{\text { final length }}{\text { intermediate length }} \cdot \frac{\text { elastic energy / final length }}{\text { intermediate area }} \\
= & \frac{\text { elastic energy } / \text { final length }}{\text { final area }} .
\end{aligned}
$$

A value of this analogue of the Cauchy stress is a linear map that coverts area in the final configuration to stress in the final configuration. Since the elastic strain-energy depends only on the elastic response $\boldsymbol{A}$, this is the true Cauchy stress for a body in which the deformation gradient is decomposed as $\boldsymbol{F}=\boldsymbol{A} \cdot \boldsymbol{G}$.

The formula above provides the form of the (Eulerian) Cauchy stress tensor for a hyperelastic body with growth, but we will need the first Piola-Kirchhoff stress tensor $\boldsymbol{P}$ to express balance laws. In hyperelasticity with growth, $\boldsymbol{P}$ remains a linear map from tangent spaces in the reference configuration to tangent spaces in the final (post-growth, post-elastic response) configuration, and it is related to the Cauchy stress tensor in the traditional manner:

$$
\begin{aligned}
\boldsymbol{P} & =(\operatorname{det} \boldsymbol{F}) \boldsymbol{T} \cdot \boldsymbol{F}^{-T} \\
& =\left(\frac{\operatorname{det} \boldsymbol{F}}{\operatorname{det} \boldsymbol{A}}\right) \boldsymbol{A} \cdot\left(\frac{\partial}{\partial \boldsymbol{A}} W(\boldsymbol{A})\right) \cdot \boldsymbol{F}^{-T} \\
& =(\operatorname{det} \boldsymbol{G}) \boldsymbol{A} \cdot\left(\frac{\partial}{\partial \boldsymbol{A}} W(\boldsymbol{A})\right) \cdot \boldsymbol{F}^{-T} .
\end{aligned}
$$

A more complete argument for importing incompatible growth into elasticity in this fashion can be found in [5]. 


\section{Reduced Plate Theory}

In a reduced theory of shells or rods, the deformations allowed are restricted to a specific class. In the case of plates (a special type of shell), for example, the body is allowed to deform to any in a family of "plate-like" configurations. This is different from a constraint such as incompressibility in that restriction to such a class of deformations cannot be expressed as a set of local constraints. With global constraints in place, weak forms of balance laws are expressed in terms of virtual displacements tangent to the (infinite-dimensional) manifold of allowed deformations. The theory is developed in [2], [10], and section of [1].

Further, it is assumed that the first Piola-Kirchhoff stress tensor can be decomposed into the sum of an active, constitutively-defined portion plus a reactive or latent portion:

$$
\boldsymbol{P}=\boldsymbol{P}_{a c t}+\boldsymbol{P}_{l a t} .
$$

$\boldsymbol{P}_{\text {lat }}$ is the portion of the stress that keeps the body plate-like, and it is assumed to do no work in the sense that

$$
\int_{(\widehat{\boldsymbol{X}} \circ \Psi)(\mathcal{B})} \boldsymbol{P}_{l a t}: \frac{\partial \stackrel{\Delta}{\boldsymbol{x}}}{\partial \boldsymbol{X}} d V=0
$$

for each virtual displacement $\stackrel{\Delta}{\boldsymbol{x}}$ tangent to the manifold of allowed configurations, where $(\widehat{\boldsymbol{X}} \circ \Psi)(\mathcal{B})$ is the reference configuration of the body. This combination of assumptions form the Global Constraint Principle. $\boldsymbol{P}_{l a t}$ is that portion of the stress that constrains the body to its plate-like configuration but performs no work in doing so. The equations of equilibrium can be expressed in weak form as

$$
\int_{(\widehat{\boldsymbol{X}} \circ \Psi)(\mathcal{B})}\left(\boldsymbol{P}_{a c t}+\boldsymbol{P}_{l a t}\right): \frac{\partial \Delta}{\partial \boldsymbol{X}} d V=\oint_{\partial(\widehat{\boldsymbol{X}} \circ \Psi)(\mathcal{B})} \boldsymbol{\tau} \cdot \stackrel{\Delta}{\boldsymbol{x}} d S,
$$

where $\partial(\widehat{\boldsymbol{X}} \circ \Psi)(\mathcal{B})$ is the boundary of the reference configuration and $\boldsymbol{\tau}$ is the applied traction vector at a point on this boundary. By Eq. (73), then, the equations of equilibrium of the constrained body have the weak form

$$
\int_{(\widehat{\boldsymbol{X}} \circ \Psi)(\mathcal{B})} \boldsymbol{P}_{a c t}: \frac{\partial \dot{\boldsymbol{x}}}{\partial \boldsymbol{X}} d V=\oint_{\partial\left(\widehat{\boldsymbol{X}}_{\circ \Psi)(\mathcal{B})}\right.} \boldsymbol{\tau} \cdot \stackrel{\Delta}{\boldsymbol{x}} d S,
$$

where $\stackrel{\Delta}{\boldsymbol{x}}$ is again a virtual displacement tangent to the manifold of allowed configurations. As pointed out in section 4 of [10], Eq. (75) will amount to an integral condition, as opposed to a stronger pointwise condition, that the active stress $\boldsymbol{P}_{\text {act }}$ will be required to satisfy on the boundary. It is through simplifications such as this that the so-called Global Constraint Principle makes nonlinear rod and shell problems manageable.

\section{The Kirchhoff Plate}

\subsection{Kirchhoff Constraints}

In a Kirchhoff plate, the final configuration is assumed to have the form

$$
(\widehat{\boldsymbol{x}} \circ f)(R, \Theta, Z)=\boldsymbol{r}(R, \Theta)+Z \boldsymbol{d}(R, \Theta),
$$


where the function $\boldsymbol{r}$ describes a two-dimensional "middle surface" of the plate, and the director field $\boldsymbol{d}$ is a unit vector-valued function that satisfies

$$
\boldsymbol{d}=\frac{\partial \boldsymbol{r}}{\partial R} \times \frac{\partial \boldsymbol{r}}{\partial \Theta} /\left\|\frac{\partial \boldsymbol{r}}{\partial R} \times \frac{\partial \boldsymbol{r}}{\partial \Theta}\right\|
$$

Thus $\boldsymbol{d}$ can be viewed as a function of $\boldsymbol{r}$ and its partial derivatives. The three-dimensional Kirchhoff plate consists of a two-dimensional surface from which one-dimensional material fibers sprout in a perpendicular direction. See Figure 7.

Momentarily viewing $\widehat{\boldsymbol{x}}$ as a function of $\boldsymbol{r}$ and its derivatives for notational convenience, we find a virtual displacement from this class of configurations has the form

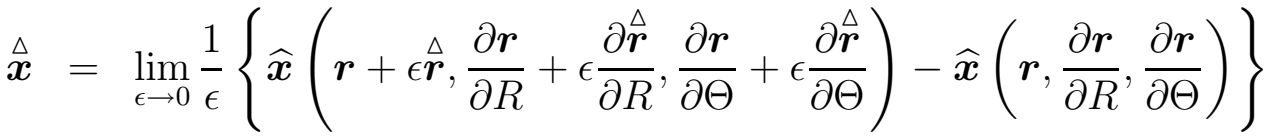

$$
\begin{aligned}
& =\lim _{\epsilon \rightarrow 0} \frac{1}{\epsilon}\left\{\left(\boldsymbol{r}+\epsilon \stackrel{\Delta}{\boldsymbol{r}}+Z \frac{\left(\boldsymbol{r}_{,}+\epsilon \hat{\boldsymbol{r}}_{,}\right) \times\left(\boldsymbol{r}_{, \Theta}+\epsilon \hat{\boldsymbol{r}}_{, \Theta}\right)}{\left\|\left(\boldsymbol{r}_{,}+\epsilon \hat{\boldsymbol{r}}_{,}\right) \times\left(\boldsymbol{r}_{, \Theta}+\epsilon \hat{\boldsymbol{r}}_{, \Theta}\right)\right\|}\right)-\left(\boldsymbol{r}+Z \frac{\boldsymbol{r}_{R} \times \boldsymbol{r}_{,}}{\left\|\boldsymbol{r},_{R} \times \boldsymbol{r}, \Theta\right\|}\right)\right\} \\
& =\stackrel{\Delta}{\boldsymbol{r}}+Z \frac{\stackrel{\Delta}{\boldsymbol{r}}, R_{R} \times \boldsymbol{r},_{\Theta}+\boldsymbol{r},_{R} \times \stackrel{\Delta}{\boldsymbol{r}}, \Theta_{,}}{\left\|\boldsymbol{r},_{R} \times \boldsymbol{r},_{\Theta}\right\|},
\end{aligned}
$$

where $\stackrel{\Delta}{\boldsymbol{r}}$ is an as-regular-as-needed function of $R$ and $\Theta$.

We could derive the vector equation of equilibrium by considering the weak form of the Method of Virtual Work with this virtual displacement, but there is an easier but less direct method. The director field must satisfy the (local) constraints

$$
\boldsymbol{d} \cdot \boldsymbol{d}=1 \quad \text { and } \quad \boldsymbol{d} \cdot \boldsymbol{r},_{\alpha}=0, \quad \alpha=R, \Theta .
$$

Encoding this into the expression $\boldsymbol{P}_{\text {act }}: \partial \Delta \overrightarrow{\boldsymbol{x}} / \partial \boldsymbol{X}$ is complicated, even in the axisymmetric case. If we introduce Lagrange multipliers $\lambda, \beta^{R}$, and $\beta^{\Theta}$, we can consider instead the integral of the following:

$$
\begin{aligned}
& \boldsymbol{\tau}^{k} \cdot(\stackrel{\Delta}{\boldsymbol{r}}+Z \stackrel{\Delta}{\boldsymbol{d}})_{, k}-\lambda \boldsymbol{d} \cdot \stackrel{\Delta}{\boldsymbol{d}}+\beta^{\alpha}\left(\stackrel{\Delta}{\boldsymbol{d}} \cdot \boldsymbol{r},,_{\alpha}+\boldsymbol{d} \cdot \stackrel{\Delta}{\boldsymbol{r}}_{, \alpha}\right) \\
& =\boldsymbol{\tau}^{k} \cdot \stackrel{\Delta}{\boldsymbol{r}}_{, k}+\beta^{\alpha} \boldsymbol{d} \cdot \boldsymbol{r},_{\alpha}+\boldsymbol{\tau}^{k} \cdot(Z \stackrel{\Delta}{\boldsymbol{d}})_{, k}+\left(\beta^{\alpha} \boldsymbol{r}_{, \alpha}-\lambda \boldsymbol{d}\right) \cdot \stackrel{\Delta}{\boldsymbol{d}} \\
& =\left(\boldsymbol{\tau}^{\alpha}+\beta^{\alpha} \boldsymbol{d}\right) \cdot \stackrel{\Delta}{\boldsymbol{r}}_{, \alpha}+\boldsymbol{\tau}^{\alpha} \cdot \ddot{\Delta}_{\boldsymbol{d}},_{\alpha}+\left(\boldsymbol{\tau}^{Z}+\beta^{\alpha} \boldsymbol{r}_{, \alpha}-\lambda \boldsymbol{d}\right) \cdot \stackrel{\Delta}{\boldsymbol{d}},
\end{aligned}
$$

where $k=R, \Theta, Z$ and $\alpha=R, \Theta$. Thanks to the Lagrange multipliers, we can treat $\stackrel{\Delta}{\boldsymbol{r}}$ and $\stackrel{\Delta}{\boldsymbol{d}}$ as though they were independent virtual displacements. We will be considering a plate with no body forces and no applied tractions, so the weak form of the equations of equilibrium will be

$$
\int_{(\widehat{\boldsymbol{X}} \circ \Psi)(\mathcal{B})}\left\{\left(\boldsymbol{\tau}^{\alpha}+\beta^{\alpha} \boldsymbol{d}\right) \cdot \stackrel{\Delta}{\boldsymbol{r}}_{,_{\alpha}}+\boldsymbol{\tau}^{\alpha} \cdot \ddot{Z}_{\boldsymbol{d}}^{,_{\alpha}}+\left(\boldsymbol{\tau}^{Z}+\beta^{\alpha} \boldsymbol{r}_{, \alpha}-\lambda \boldsymbol{d}\right) \cdot \stackrel{\Delta}{\boldsymbol{d}}\right\} d V=0
$$


for all admissible virtual displacements $\boldsymbol{r}$.

Note that

$$
\begin{aligned}
& \boldsymbol{\tau}^{R} \cdot(\stackrel{\Delta}{\boldsymbol{r}}+Z \stackrel{\Delta}{\boldsymbol{d}}),_{R}=\left(\boldsymbol{P}_{a c t} \cdot \boldsymbol{E}^{R}\right) \cdot \frac{\partial}{\partial R}(\stackrel{\Delta}{\boldsymbol{r}}+Z \stackrel{\Delta}{\boldsymbol{d}}) \\
& =\left(\boldsymbol{P}_{a c t} \cdot \boldsymbol{h}_{1}\right) \cdot \frac{\partial}{\partial R}(\stackrel{\Delta}{\boldsymbol{r}}+Z \stackrel{\Delta}{\boldsymbol{d}}) \\
& \boldsymbol{\tau}^{\Theta} \cdot(\stackrel{\Delta}{\boldsymbol{r}}+Z \stackrel{\Delta}{\boldsymbol{d}}){ }_{, \Theta}=\left(\boldsymbol{P}_{a c t} \cdot \boldsymbol{E}^{\Theta}\right) \cdot \frac{\partial}{\partial \Theta}(\stackrel{\Delta}{\boldsymbol{r}}+Z \stackrel{\Delta}{\boldsymbol{d}}) \\
& =\left(\boldsymbol{P}_{a c t} \cdot \frac{\boldsymbol{h}_{2}}{R}\right) \cdot \frac{\partial}{\partial \Theta}(\stackrel{\Delta}{\boldsymbol{r}}+Z \stackrel{\Delta}{\boldsymbol{d}}) \text {. }
\end{aligned}
$$

\subsection{Kinematics of an Axisymmetric Kirchhoff Plate}

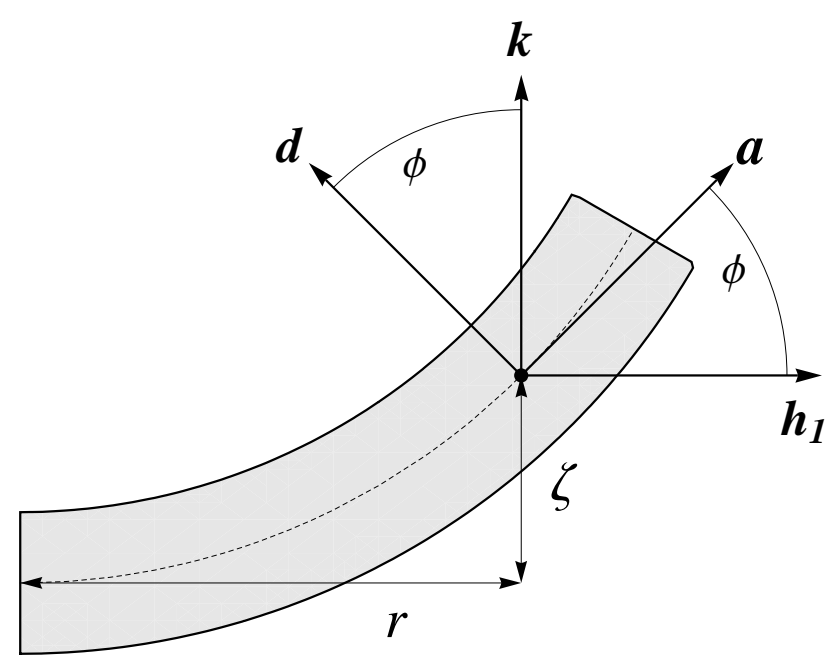

Figure 7: A radial slice of an axisymmetric Kirchhoff plate. $\zeta$ is the height of a point of the middle surface, and a material point's height above the middle surface is measured along the unit vector $\boldsymbol{b}=\boldsymbol{d}$, which is perpendicular to the middle surface.

We consider axisymmetric deformations of an axisymmetric Kirchhoff plate. The reference configuration has the form

$$
\widehat{\boldsymbol{X}}(R, \Theta, Z)=R \cos (\Theta) \boldsymbol{i}+R \sin (\Theta) \boldsymbol{j}+Z \boldsymbol{k}=R \boldsymbol{h}_{1}(\Theta)+Z \boldsymbol{k}
$$

where

$$
\boldsymbol{h}_{1}(\Theta)=\cos (\Theta) \boldsymbol{i}+\sin (\Theta) \boldsymbol{j} .
$$

As a function of $(R, \Theta, Z)$, the final configuration will have the form

$$
(\widehat{\boldsymbol{x}} \circ f)(R, \Theta, Z)=\underbrace{r(R) \boldsymbol{h}_{1}(\Theta)+\zeta(R) \boldsymbol{k}}_{\boldsymbol{r}(R, \Theta)}+Z \boldsymbol{d}(R, \Theta) .
$$


Here $r$ is the cylindrical radius of a point in the middle surface, and $\zeta$ is the height of a point in the middle surface. See Figure 7.

An outward-pointing radial tangent vector to the middle surface is

$$
\frac{d}{d R}\left(r(R) \boldsymbol{h}_{1}(\Theta)+\zeta(R) \boldsymbol{k}\right)=r^{\prime}(R) \boldsymbol{h}_{1}(\Theta)+\zeta^{\prime}(R) \boldsymbol{k} .
$$

We define $\boldsymbol{a}$ to be the corresponding unit vector:

$$
\boldsymbol{a}=\frac{r^{\prime} \boldsymbol{h}_{1}+\zeta^{\prime} \boldsymbol{k}}{\sqrt{\left(r^{\prime}\right)^{2}+\left(\zeta^{\prime}\right)^{2}}}=\cos \phi \boldsymbol{h}_{1}+\sin \phi \boldsymbol{k},
$$

where $\phi$ is the angle formed by $\boldsymbol{a}$ and the radial unit vector $\boldsymbol{h}_{1}$ :

$$
\phi=\arctan \left(\frac{\zeta^{\prime}}{r^{\prime}}\right) \text {. }
$$

The director $\boldsymbol{d}$ is the "upward"-pointing unit vector orthogonal to $\boldsymbol{a}$ :

$$
\boldsymbol{d}=-\sin \phi \boldsymbol{h}_{1}+\cos \phi \boldsymbol{k}
$$

The azimuthal unit vector $\boldsymbol{h}_{2}$ is the third member of the local orthonormal basis.

To construct the deformation gradient, we appeal to the relation

$$
\frac{\partial}{\partial \xi^{i}}(\chi \circ \widehat{\boldsymbol{X}})\left(\xi^{1}, \xi^{2}, \xi^{3}\right)=\boldsymbol{F} \cdot \boldsymbol{E}_{i}
$$

where $\boldsymbol{F}$ and the $\boldsymbol{E}_{i}$ are evaluated at $\widehat{\boldsymbol{X}}\left(\xi^{1}, \xi^{2}, \xi^{3}\right)$.

$$
\begin{aligned}
& \frac{\partial}{\partial R}\left(r(R) \boldsymbol{h}_{1}(\Theta)+\zeta(R) \boldsymbol{k}+Z \boldsymbol{d}(R, \Theta)\right)=r^{\prime}(R) \boldsymbol{h}_{1}(\Theta)+\zeta^{\prime}(R) \boldsymbol{k}+Z \frac{\partial \boldsymbol{d}}{\partial R}(R, \Theta) \\
& =r^{\prime} \boldsymbol{h}_{1}+\zeta^{\prime} \boldsymbol{k}-Z \phi^{\prime}\left(\cos \phi \boldsymbol{h}_{1}+\sin \phi \boldsymbol{k}\right) \\
& =r^{\prime} \boldsymbol{h}_{1}+\zeta^{\prime} \boldsymbol{k}-Z \phi^{\prime} \boldsymbol{a} \text {. } \\
& \frac{\partial}{\partial \Theta}\left(r(R) \boldsymbol{h}_{1}(\Theta)+\zeta(R) \boldsymbol{k}+Z \boldsymbol{d}(R, \Theta)\right)=r(R) \boldsymbol{h}_{2}(\Theta)+Z \frac{\partial \boldsymbol{d}}{\partial \Theta}(R, \Theta) \\
& =r \boldsymbol{h}_{2}-Z \sin \phi \frac{\partial \boldsymbol{h}_{1}}{\partial \Theta} \\
& =(r-Z \sin \phi) \boldsymbol{h}_{2} \text {. } \\
& \frac{\partial}{\partial Z}\left(r(R) \boldsymbol{h}_{1}(\Theta)+\zeta(R) \boldsymbol{k}+Z \boldsymbol{d}(R, \Theta)\right)=\boldsymbol{d}(R, \Theta)
\end{aligned}
$$

These results show that

$$
\begin{aligned}
\boldsymbol{F} & =\left(r^{\prime} \boldsymbol{h}_{1}+\zeta^{\prime} \boldsymbol{k}-Z \phi^{\prime} \boldsymbol{a}\right) \otimes \boldsymbol{E}^{R}+(r-Z \sin \phi) \boldsymbol{h}_{2} \otimes \boldsymbol{E}^{\Theta}+\boldsymbol{d} \otimes \boldsymbol{E}^{Z} \\
& =\left(r^{\prime} \boldsymbol{h}_{1}+\zeta^{\prime} \boldsymbol{k}-Z \phi^{\prime} \boldsymbol{a}\right) \otimes \boldsymbol{h}_{1}+\left(\frac{r-Z \sin \phi}{R}\right) \boldsymbol{h}_{2} \otimes \boldsymbol{h}_{2}+\boldsymbol{d} \otimes \boldsymbol{k}
\end{aligned}
$$


where it must be understood that the vectors on the right-hand sides of the tensor products are anchored at the reference point, while those on the left-hand sides are anchored at the deformed point.

We will find it convenient to use the orthonormal basis $\left\{\boldsymbol{a}, \boldsymbol{h}_{2}, \boldsymbol{d}\right\}$ in the deformed configuration, so we will re-write the left-hand side of the first tensor product in $\boldsymbol{F}$. Note that

$$
\left(\begin{array}{l}
\boldsymbol{a} \\
\boldsymbol{d}
\end{array}\right)=\left(\begin{array}{cc}
\cos \phi & \sin \phi \\
-\sin \phi & \cos \phi
\end{array}\right)\left(\begin{array}{c}
\boldsymbol{h}_{1} \\
\boldsymbol{k}
\end{array}\right) \Longrightarrow\left(\begin{array}{c}
\boldsymbol{h}_{1} \\
\boldsymbol{k}
\end{array}\right)=\left(\begin{array}{cc}
\cos \phi & -\sin \phi \\
\sin \phi & \cos \phi
\end{array}\right)\left(\begin{array}{l}
\boldsymbol{a} \\
\boldsymbol{d}
\end{array}\right)
$$

As a result,

$$
\begin{aligned}
r^{\prime} \boldsymbol{h}_{1}+\zeta^{\prime} \boldsymbol{k} & =r^{\prime}(\cos \phi \boldsymbol{a}-\sin \phi \boldsymbol{d})+\zeta^{\prime}(\sin \phi \boldsymbol{a}+\cos \phi \boldsymbol{d}) \\
& =\left(r^{\prime} \cos \phi+\zeta^{\prime} \sin \phi\right) \boldsymbol{a}+\left(\zeta^{\prime} \cos \phi-r^{\prime} \sin \phi\right) \boldsymbol{d}
\end{aligned}
$$

By definition of $\phi$, though, $\zeta^{\prime}=r^{\prime} \tan \phi$, so

$$
\begin{aligned}
r^{\prime} \cos \phi+\zeta^{\prime} \sin \phi & =r^{\prime}(\cos \phi+\tan \phi \sin \phi) \\
& =r^{\prime}\left(\cos \phi+\frac{\sin ^{2} \phi}{\cos \phi}\right) \\
& =r^{\prime} \sec \phi \\
\zeta^{\prime} \cos \phi-r^{\prime} \sin \phi & =r^{\prime}(\tan \phi \cos \phi-\sin \phi)=0 .
\end{aligned}
$$

The deformation gradient can thus be written as

$$
\boldsymbol{F}=\left(r^{\prime} \sec \phi-Z \phi^{\prime}\right) \boldsymbol{a} \otimes \boldsymbol{h}_{1}+\left(\frac{r-Z \sin \phi}{R}\right) \boldsymbol{h}_{2} \otimes \boldsymbol{h}_{2}+\boldsymbol{d} \otimes \boldsymbol{k}
$$

We will need the following expression for later computations:

$$
\boldsymbol{F}^{-T}=\left(r^{\prime} \sec \phi-Z \phi^{\prime}\right)^{-1} \boldsymbol{a} \otimes \boldsymbol{h}_{1}+\left(\frac{r-Z \sin \phi}{R}\right)^{-1} \boldsymbol{h}_{2} \otimes \boldsymbol{h}_{2}+\boldsymbol{d} \otimes \boldsymbol{k} .
$$

We will use incompatible growth tensors of the form presented in Eq. (44):

$$
\begin{aligned}
\boldsymbol{G} & =\gamma_{1} \boldsymbol{E}_{r} \otimes \boldsymbol{E}^{R}+\frac{\gamma_{2}}{\gamma_{1}} \boldsymbol{E}_{\theta} \otimes \boldsymbol{E}^{\Theta}+\boldsymbol{E}_{z} \otimes \boldsymbol{E}^{Z} \\
& =\gamma_{1} \boldsymbol{h}_{1} \otimes \boldsymbol{h}_{1}+\frac{\gamma_{2}}{\gamma_{1}} r \boldsymbol{h}_{2} \otimes \frac{\boldsymbol{h}_{2}}{R}+\boldsymbol{k} \otimes \boldsymbol{k} \\
& =\gamma_{1} \boldsymbol{h}_{1} \otimes \boldsymbol{h}_{1}+\gamma_{2} \boldsymbol{h}_{2} \otimes \boldsymbol{h}_{2}+\boldsymbol{k} \otimes \boldsymbol{k}, \quad\left(r=\gamma_{1} R\right)
\end{aligned}
$$

with $\gamma_{1}$ and $\gamma_{2}$ constant. It should be noted that in Eq. (102) the vectors on the right-hand side of each tensor product are anchored at a point in the reference configuration and each vector on the left-hand side of a tensor product is anchored at the image under the incompatible growth discussed above. 
With $\boldsymbol{F}$ and $\boldsymbol{G}$ so defined, the tensor describing the elastic response to growth is

$$
\begin{aligned}
\boldsymbol{A} & =\boldsymbol{F} \cdot \boldsymbol{G}^{-1} \\
& =\left(\frac{r^{\prime} \sec \phi-Z \phi^{\prime}}{\gamma_{1}}\right) \boldsymbol{a} \otimes \boldsymbol{h}_{1}+\left(\frac{r-Z \sin \phi}{\gamma_{2} R}\right) \boldsymbol{h}_{2} \otimes \boldsymbol{h}_{2}+\boldsymbol{d} \otimes \boldsymbol{k} .
\end{aligned}
$$

The vector on the right-hand side of each tensor product is anchored at a point in the incompatibly grown state, and each vector on the left-hand side of a tensor product is anchored at a point in the final configuration.

The most important tensor for the constitutive relation will be the Eulerian tensor

$$
\boldsymbol{A} \cdot \boldsymbol{A}^{T}=\left(\frac{r^{\prime} \sec \phi-Z \phi^{\prime}}{\gamma_{1}}\right)^{2} \boldsymbol{a} \otimes \boldsymbol{a}+\left(\frac{r-Z \sin \phi}{\gamma_{2} R}\right)^{2} \boldsymbol{h}_{2} \otimes \boldsymbol{h}_{2}+\boldsymbol{d} \otimes \boldsymbol{d} .
$$

We now consider variations with $\stackrel{\Delta}{\boldsymbol{r}}$. We start with the following expression

$$
\begin{aligned}
\int_{\text {plate }}\left(\boldsymbol{\tau}^{\alpha}+\beta^{\alpha} \boldsymbol{d}\right) \cdot \stackrel{\Delta}{\boldsymbol{r}}_{, \alpha} d V & =\int_{\text {plate }}\left\{\left(\boldsymbol{P}_{a c t} \cdot \boldsymbol{h}_{1}+\beta^{R} \boldsymbol{d}\right) \cdot \frac{\partial \overrightarrow{\boldsymbol{r}}}{\partial R}+\left(\boldsymbol{P}_{a c t} \cdot \frac{\boldsymbol{h}_{2}}{R}+\beta^{\Theta} \boldsymbol{d}\right) \cdot \frac{\partial \stackrel{\Delta}{\partial \Theta}\} d V}{}\right. \\
& =\int_{\text {plate }}\left\{\frac{\partial \overrightarrow{\boldsymbol{r}}}{\partial R} \cdot\left(\boldsymbol{P}_{a c t} \cdot \boldsymbol{h}_{1}+\beta^{R} \boldsymbol{d}\right)+\frac{\partial \overrightarrow{\boldsymbol{r}}}{\partial \Theta} \cdot\left(\boldsymbol{P}_{a c t} \cdot \frac{\boldsymbol{h}_{2}}{R}+\beta^{\Theta} \boldsymbol{d}\right)\right\} d V
\end{aligned}
$$

It will be helpful to consider $\beta^{R}$ and $\beta^{\Theta}$ as components of a vector $\boldsymbol{\beta}$ :

$$
\boldsymbol{\beta}=\beta^{R} \boldsymbol{E}_{R}+\beta^{\Theta} \boldsymbol{E}_{\Theta}=\beta^{R} \boldsymbol{h}_{1}+\beta^{\Theta} R \boldsymbol{h}_{2} .
$$

With $\boldsymbol{\beta}$ so defined, we can consider the two-point tensor field $\boldsymbol{P}_{a c t}+\boldsymbol{d} \otimes \boldsymbol{\beta}$.

Consider the planar portion of $\stackrel{\Delta}{\boldsymbol{r}} \cdot\left(\boldsymbol{P}_{a c t}+\boldsymbol{d} \otimes \boldsymbol{\beta}\right)$ :

$$
\begin{aligned}
\stackrel{\Delta}{\boldsymbol{r}} \cdot\left(\boldsymbol{P}_{a c t}+\boldsymbol{d} \otimes \boldsymbol{\beta}\right)_{\text {planar }} & =\left(\stackrel{\Delta}{\boldsymbol{r}} \cdot \boldsymbol{P}_{a c t} \cdot \boldsymbol{h}_{1}+(\stackrel{\Delta}{\boldsymbol{r}} \cdot \boldsymbol{d})\left(\boldsymbol{\beta} \cdot \boldsymbol{h}_{1}\right)\right) \boldsymbol{h}_{1} \\
& +\left(\stackrel{\Delta}{\boldsymbol{r}} \cdot \boldsymbol{P}_{a c t} \cdot \boldsymbol{h}_{2}+(\stackrel{\Delta}{\boldsymbol{r}} \cdot \boldsymbol{d})\left(\boldsymbol{\beta} \cdot \boldsymbol{h}_{2}\right)\right) \boldsymbol{h}_{2} .
\end{aligned}
$$

In polar coordinates, the divergence of a planar vector field is

$$
\operatorname{Div}\left(f \boldsymbol{h}_{1}+g \boldsymbol{h}_{2}\right)=\frac{1}{R} \frac{\partial}{\partial R}(R f)+\frac{1}{R} \frac{\partial g}{\partial \Theta},
$$


so the divergence of $\stackrel{\Delta}{\boldsymbol{r}} \cdot\left(\boldsymbol{P}_{a c t}+\boldsymbol{d} \otimes \boldsymbol{\beta}\right)_{\text {planar }}$ is

$$
\begin{aligned}
& \operatorname{Div}\left(\stackrel{\Delta}{\boldsymbol{r}} \cdot\left(\boldsymbol{P}_{a c t}+\boldsymbol{d} \otimes \boldsymbol{\beta}\right)_{\mathrm{planar}}\right)=\frac{1}{R} \frac{\partial}{\partial R}\left(R \stackrel{\Delta}{\boldsymbol{r}} \cdot \boldsymbol{P}_{a c t} \cdot \boldsymbol{h}_{1}+R(\stackrel{\Delta}{\boldsymbol{r}} \cdot \boldsymbol{d})\left(\boldsymbol{\beta} \cdot \boldsymbol{h}_{1}\right)\right) \\
& +\frac{1}{R} \frac{\partial}{\partial \Theta}\left(\stackrel{\Delta}{\boldsymbol{r}} \cdot \boldsymbol{P}_{a c t} \cdot \boldsymbol{h}_{2}+(\stackrel{\Delta}{\boldsymbol{r}} \cdot \boldsymbol{d})\left(\boldsymbol{\beta} \cdot \boldsymbol{h}_{2}\right)\right) \\
& =\frac{\partial \overrightarrow{\boldsymbol{r}}}{\partial R} \cdot\left(\boldsymbol{P}_{a c t} \cdot \boldsymbol{h}_{1}+\beta^{R} \boldsymbol{d}\right)+\stackrel{\Delta}{\boldsymbol{r}} \cdot \frac{1}{R} \frac{\partial}{\partial R}\left(R \boldsymbol{P}_{a c t} \cdot \boldsymbol{h}_{1}+R \beta^{R} \boldsymbol{d}\right) \\
& +\frac{1}{R} \frac{\partial \dot{\boldsymbol{r}}}{\partial \Theta} \cdot\left(\boldsymbol{P}_{a c t} \cdot \boldsymbol{h}_{2}+\left(\boldsymbol{\beta} \cdot \boldsymbol{h}_{2}\right) \boldsymbol{d}\right)+\stackrel{\Delta}{\boldsymbol{r}} \cdot \frac{1}{R} \frac{\partial}{\partial \Theta}\left(\boldsymbol{P}_{a c t} \cdot \boldsymbol{h}_{2}+\left(\boldsymbol{\beta} \cdot \boldsymbol{h}_{2}\right) \boldsymbol{d}\right) \\
& =\frac{\partial \stackrel{\Delta}{\partial}}{\partial R} \cdot\left(\boldsymbol{P}_{a c t} \cdot \boldsymbol{h}_{1}+\beta^{R} \boldsymbol{d}\right)+\stackrel{\Delta}{\boldsymbol{r}} \cdot \frac{1}{R} \frac{\partial}{\partial R}\left(R \boldsymbol{P}_{a c t} \cdot \boldsymbol{h}_{1}+R \beta^{R} \boldsymbol{d}\right) \\
& +\frac{1}{R} \frac{\partial \dot{\boldsymbol{r}}}{\partial \Theta} \cdot\left(\boldsymbol{P}_{a c t} \cdot \boldsymbol{h}_{2}+\beta^{\Theta} R \boldsymbol{d}\right)+\stackrel{\Delta}{\boldsymbol{r}} \cdot \frac{1}{R} \frac{\partial}{\partial \Theta}\left(\boldsymbol{P}_{a c t} \cdot \boldsymbol{h}_{2}+\beta^{\Theta} R \boldsymbol{d}\right) \\
& =\frac{\partial \hat{\boldsymbol{r}}}{\partial R} \cdot\left(\boldsymbol{P}_{a c t} \cdot \boldsymbol{h}_{1}+\beta^{R} \boldsymbol{d}\right)+\stackrel{\Delta}{\boldsymbol{r}} \cdot \frac{1}{R} \frac{\partial}{\partial R}\left(R \boldsymbol{P}_{a c t} \cdot \boldsymbol{h}_{1}+R \beta^{R} \boldsymbol{d}\right) \\
& +\frac{\partial \stackrel{\Delta}{r}}{\partial \Theta} \cdot\left(\boldsymbol{P}_{a c t} \cdot \frac{\boldsymbol{h}_{2}}{R}+\beta^{\Theta} \boldsymbol{d}\right)+\stackrel{\Delta}{\boldsymbol{r}} \cdot \frac{\partial}{\partial \Theta}\left(\boldsymbol{P}_{a c t} \cdot \frac{\boldsymbol{h}_{2}}{R}+\beta^{\Theta} \boldsymbol{d}\right) .
\end{aligned}
$$

The $\stackrel{\Delta}{\boldsymbol{r}}$ portion of Eq. (81) can be written as

$$
\begin{aligned}
& \int_{\text {plate }}\left\{\frac{\partial \overrightarrow{\boldsymbol{r}}}{\partial R} \cdot\left(\boldsymbol{P}_{a c t} \cdot \boldsymbol{h}_{1}+\beta^{R} \boldsymbol{d}\right)+\frac{\partial \dot{\boldsymbol{r}}}{\partial \Theta} \cdot\left(\boldsymbol{P}_{a c t} \cdot \frac{\boldsymbol{h}_{2}}{R}+\beta^{\Theta} \boldsymbol{d}\right)\right\} d V \\
= & \int_{\text {plate }} \operatorname{Div}\left(\stackrel{\Delta}{\boldsymbol{r}} \cdot\left(\boldsymbol{P}_{a c t}+\boldsymbol{d} \otimes \boldsymbol{\beta}\right)_{\text {planar }}\right) d V \\
- & \int_{\text {plate }} \stackrel{\Delta}{\boldsymbol{r}} \cdot\left\{\frac{1}{R} \frac{\partial}{\partial R}\left(R \boldsymbol{P}_{a c t} \cdot \boldsymbol{h}_{1}+R \beta^{R} \boldsymbol{d}\right)+\frac{\partial}{\partial \Theta}\left(\boldsymbol{P}_{a c t} \cdot \frac{\boldsymbol{h}_{2}}{R}+\beta^{\Theta} \boldsymbol{d}\right)\right\} d V .
\end{aligned}
$$

By the Divergence Theorem in the plane, the integral of the first integrand can be re-written

$$
\begin{aligned}
& \int_{\text {plate }} \operatorname{Div}\left(\stackrel{\Delta}{\boldsymbol{r}} \cdot\left(\boldsymbol{P}_{a c t}+\boldsymbol{d} \otimes \boldsymbol{\beta}\right)_{\text {planar }}\right) d v \\
& =\int_{H_{1}}^{H_{2}}\left\{\int_{\text {slice }} \operatorname{Div}\left(\stackrel{\Delta}{\boldsymbol{r}} \cdot\left(\boldsymbol{P}_{\text {act }}+\boldsymbol{d} \otimes \boldsymbol{\beta}\right)_{\text {planar }}\right) R d R d \Theta\right\} d Z \\
& =\int_{H_{1}}^{H_{2}}\left\{\oint_{\partial(\text { slice })}\left(\stackrel{\Delta}{\boldsymbol{r}} \cdot\left(\boldsymbol{P}_{a c t}+\boldsymbol{d} \otimes \boldsymbol{\beta}\right)_{\text {planar }}\right) \cdot \boldsymbol{\nu} d \ell\right\} d Z \\
& =\oint_{\partial(\text { slice })} \stackrel{\Delta}{\boldsymbol{r}} \cdot\left\{\int_{H_{1}}^{H_{2}}\left(\boldsymbol{P}_{a c t}+\boldsymbol{d} \otimes \boldsymbol{\beta}\right)_{\text {planar }} \cdot \boldsymbol{\nu} d Z\right\} d \ell,
\end{aligned}
$$

where the "slice" mentioned is a planar slice of the reference configuration of the plate, $\partial$ (slice) is the curve that forms the planar boundary of the slice, $\boldsymbol{\nu}$ is the outward-pointing unit normal on $\partial$ (slice), and $d \ell$ is arclength measure on $\partial$ (slice). 
In order to balance the boundary term in the weak form of the Principle of Virtual Work, we must specify the value of

$$
\int_{H_{1}}^{H_{2}}\left(\boldsymbol{P}_{a c t}+\boldsymbol{d} \otimes \boldsymbol{\beta}\right)_{\mathrm{planar}} \cdot \boldsymbol{\nu} d Z=\int_{H_{1}}^{H_{2}}\left(\boldsymbol{P}_{a c t}+\boldsymbol{d} \otimes \boldsymbol{\beta}\right) \cdot \boldsymbol{h}_{1} d Z
$$

on the boundary of the middle surface.

Now that boundary terms are balanced, we have

$$
\int_{\text {plate }} \stackrel{\Delta}{\boldsymbol{r}} \cdot\left\{\frac{1}{R} \frac{\partial}{\partial R}\left(R \boldsymbol{P}_{a c t} \cdot \boldsymbol{h}_{1}+R \beta^{R} \boldsymbol{d}\right)+\frac{\partial}{\partial \Theta}\left(\boldsymbol{P}_{a c t} \cdot \frac{\boldsymbol{h}_{2}}{R}+\beta^{\Theta} \boldsymbol{d}\right)\right\} d V=0
$$

for all as-smooth-as-needed function $\stackrel{\Delta}{\boldsymbol{r}}$ of $R$ and $\Theta$. Since

$$
\begin{aligned}
& \int_{\text {plate }} \stackrel{\Delta}{\boldsymbol{r}} \cdot\left\{\frac{1}{R} \frac{\partial}{\partial R}\left(R \boldsymbol{P}_{a c t} \cdot \boldsymbol{h}_{1}+R \beta^{R} \boldsymbol{d}\right)+\frac{\partial}{\partial \Theta}\left(\boldsymbol{P}_{a c t} \cdot \frac{\boldsymbol{h}_{2}}{R}+\beta^{\Theta} \boldsymbol{d}\right)\right\} d v \\
= & \int_{\text {slice }} \int_{H_{1}}^{H_{2}}\left\{\frac{1}{R} \frac{\partial}{\partial R}\left(R \boldsymbol{P}_{a c t} \cdot \boldsymbol{h}_{1}+R \beta^{R} \boldsymbol{d}\right)+\frac{\partial}{\partial \Theta}\left(\boldsymbol{P}_{a c t} \cdot \frac{\boldsymbol{h}_{2}}{R}+\beta^{\Theta} \boldsymbol{d}\right)\right\} d Z \cdot \stackrel{\boldsymbol{r}}{R} d R d \Theta \\
= & 0
\end{aligned}
$$

for such a large class of $\stackrel{\Delta}{\boldsymbol{r}}$, we conclude that the integral in $Z$ is identically zero:

$$
\int_{H_{1}}^{H_{2}}\left\{\frac{1}{R} \frac{\partial}{\partial R}\left(R \boldsymbol{P}_{a c t} \cdot \boldsymbol{h}_{1}+R \beta^{R} \boldsymbol{d}\right)+\frac{\partial}{\partial \Theta}\left(\boldsymbol{P}_{a c t} \cdot \frac{\boldsymbol{h}_{2}}{R}+\beta^{\Theta} \boldsymbol{d}\right)\right\} d Z=\mathbf{0 .}
$$

Next, we consider the variation in $\stackrel{\Delta}{\boldsymbol{d}}$. We apply the same procedure as for the variation in $\stackrel{\Delta}{\boldsymbol{r}}$. The result will be a weak version of the balance of angular momentum in the constrained plate with zero applied moments.

$$
\begin{aligned}
& \int_{\text {plate }}\left\{\boldsymbol{\tau}^{\alpha} \cdot Z \stackrel{\Delta}{\boldsymbol{d}}_{\alpha}+\left(\boldsymbol{\tau}^{Z}+\beta^{\alpha} \boldsymbol{r},,_{\alpha}-\lambda \boldsymbol{d}\right) \cdot \stackrel{\Delta}{\boldsymbol{d}}\right\} d V \\
& =\int_{\text {plate }}\left\{\boldsymbol{\tau}^{R} \cdot Z \frac{\partial \dot{\boldsymbol{d}}}{\partial R}+\boldsymbol{\tau}^{\Theta} \cdot Z \frac{\partial \stackrel{\Delta}{\boldsymbol{d}}}{\partial \Theta}+\left(\boldsymbol{\tau}^{Z}+\beta^{\alpha} \boldsymbol{r}_{, \alpha}-\lambda \boldsymbol{d}\right) \cdot \stackrel{\Delta}{\boldsymbol{d}}\right\} d V \\
& =\int_{\text {plate }} Z \operatorname{Div}\left(\stackrel{\Delta}{\boldsymbol{d}} \cdot \boldsymbol{P}_{\text {act }}\right)_{\text {planar }} d V \\
& +\int_{\text {plate }} \stackrel{\Delta}{\boldsymbol{d}} \cdot\left\{\left(\boldsymbol{\tau}^{Z}+\beta^{\alpha} \boldsymbol{r}_{\alpha}-\lambda \boldsymbol{d}\right)-Z \frac{1}{R} \frac{\partial}{\partial R}\left(R \boldsymbol{P}_{a c t} \cdot \boldsymbol{h}_{1}\right)-Z \frac{\partial}{\partial \Theta}\left(\boldsymbol{P}_{a c t} \cdot \frac{\boldsymbol{h}_{2}}{R}\right)\right\} d V \\
& =\oint_{\partial \text { (slice) }} \stackrel{\Delta}{\boldsymbol{d}} \cdot\left(\int_{H_{1}}^{H_{2}} Z \boldsymbol{P}_{a c t} d Z\right) \cdot \boldsymbol{\nu} d \ell \\
& +\int_{\text {slice }} \stackrel{\Delta}{\boldsymbol{d}} \cdot \int_{H_{1}}^{H_{2}}\left\{\boldsymbol{\tau}^{Z}+\beta^{\alpha} \boldsymbol{r},,_{\alpha}-\lambda \boldsymbol{d}-Z \frac{1}{R} \frac{\partial}{\partial R}\left(R \boldsymbol{P}_{a c t} \cdot \boldsymbol{h}_{1}\right)-Z \frac{\partial}{\partial \Theta}\left(\boldsymbol{P}_{a c t} \cdot \frac{\boldsymbol{h}_{2}}{R}\right)\right\} d Z R d R d \theta \text {. }
\end{aligned}
$$


As before, $\boldsymbol{\nu}=\boldsymbol{h}_{1}$ is the outward-pointing unit normal to the planar boundary $\partial$ (slice) of the slice in the reference configuration, and $d \ell$ is arclength measure on $\partial$ (slice).

To balance the boundary terms, we must set values of the moment

$$
\int_{H_{1}}^{H_{2}} Z\left(\boldsymbol{P}_{a c t} \cdot \boldsymbol{h}_{1}\right) d Z
$$

on the planar boundary of the middle surface.

We have found that

$\int_{\text {slice }} \stackrel{\Delta}{\boldsymbol{d}} \cdot \int_{H_{1}}^{H_{2}}\left\{\boldsymbol{\tau}^{Z}+\beta^{\alpha} \boldsymbol{r},,_{\alpha}-\lambda \boldsymbol{d}-Z \frac{1}{R} \frac{\partial}{\partial R}\left(R \boldsymbol{P}_{a c t} \cdot \boldsymbol{h}_{1}\right)-Z \frac{\partial}{\partial \Theta}\left(\boldsymbol{P}_{a c t} \cdot \frac{\boldsymbol{h}_{2}}{R}\right)\right\} d Z R d R d \theta=0$

for all as-smooth-as-needed functions $\stackrel{\Delta}{\boldsymbol{d}}$ of $R$ and $\Theta$. We conclude that

$$
\int_{H_{1}}^{H_{2}}\left\{\boldsymbol{\tau}^{Z}+\beta^{\alpha} \boldsymbol{r}_{,_{\alpha}}-\lambda \boldsymbol{d}-Z \frac{1}{R} \frac{\partial}{\partial R}\left(R \boldsymbol{P}_{a c t} \cdot \boldsymbol{h}_{1}\right)-Z \frac{\partial}{\partial \Theta}\left(\boldsymbol{P}_{a c t} \cdot \frac{\boldsymbol{h}_{2}}{R}\right)\right\} d Z=\mathbf{0} .
$$

\subsection{Addressing Lagrange Multipliers}

We are not yet ready to introduce the constitutive relation to close the system of equations. The quantities $\lambda, \beta^{R}$, and $\beta^{\Theta}$ are not constitutively defined. However, there is enough information in the equations above to get around this complication.

First we claim that if we take the cross-product with $\boldsymbol{d}$, the term $\lambda \boldsymbol{d}$ will vanish. By the form of $\boldsymbol{G}$ we are assuming and the form of $\boldsymbol{F}^{-T}$ found above, we can be sure that $\boldsymbol{F}^{-T} \cdot \boldsymbol{k}=\boldsymbol{d}$. If we use an isotropic hyperelastic constitutive relation, then the form of $\boldsymbol{A}=\boldsymbol{F} \cdot \boldsymbol{G}^{-1}$ in such a relation will guarantee that $\boldsymbol{T} \cdot \boldsymbol{d}$ points in the direction $\boldsymbol{d}$. As a result,

$$
\begin{aligned}
\int_{H_{1}}^{H_{2}} \boldsymbol{\tau}^{Z} d Z & =\int_{H_{1}}^{H_{2}}\left(\boldsymbol{P}_{a c t} \cdot \boldsymbol{k}\right) d Z \\
& =\int_{H_{1}}^{H_{2}}(\operatorname{det} \boldsymbol{F})\left(\boldsymbol{T} \cdot \boldsymbol{F}^{-T} \cdot \boldsymbol{k}\right) d Z \\
& =\int_{H_{1}}^{H_{2}}(\operatorname{det} \boldsymbol{F})(\boldsymbol{T} \cdot \boldsymbol{d}) d Z \propto \boldsymbol{d}
\end{aligned}
$$

which implies

$$
\boldsymbol{d} \times \int_{H_{1}}^{H_{2}} \boldsymbol{\tau}^{Z} d Z=\mathbf{0}
$$

the elimination we sought. 
When we take the cross-product of $\boldsymbol{d}$ with Eq. (118), we have

$$
\begin{aligned}
\boldsymbol{d} \times \frac{1}{R} \frac{\partial}{\partial R}\left(R \int_{H_{1}}^{H_{2}} Z\left(\boldsymbol{P}_{a c t} \cdot \boldsymbol{h}_{1}\right) d Z\right)+\boldsymbol{d} \times \frac{\partial}{\partial \Theta} \int_{H_{1}}^{H_{2}} Z\left(\boldsymbol{P}_{a c t} \cdot \frac{\boldsymbol{h}_{2}}{R}\right) d Z & \\
-\boldsymbol{d} \times \boldsymbol{r}, \int_{H_{1}} \int^{H_{2}} \beta^{\alpha} d Z & =\mathbf{0 .}
\end{aligned}
$$

We will consider all these terms in detail to find expressions for $\beta^{R}$ and $\beta^{\Theta}$ in terms of kinematic and constitutively-defined quantities.

By the symmetry of the deformation, the vector field $\boldsymbol{P}_{a c t} \cdot \boldsymbol{h}_{1}$ has zero projection onto $\boldsymbol{h}_{2}$. It is determined solely by its projections onto $\boldsymbol{h}_{1}$ and $\boldsymbol{k}$ :

$$
\left(\boldsymbol{P}_{a c t} \cdot \boldsymbol{h}_{1}\right)=\left(\boldsymbol{h}_{1} \cdot \boldsymbol{P}_{a c t} \cdot \boldsymbol{h}_{1}\right) \boldsymbol{h}_{1}+\left(\boldsymbol{k} \cdot \boldsymbol{P}_{a c t} \cdot \boldsymbol{h}_{1}\right) \boldsymbol{k}
$$

Since $\boldsymbol{h}_{1}$ and $\boldsymbol{k}$ are $R$-independent,

$$
\frac{1}{R} \frac{\partial}{\partial R} R\left(\boldsymbol{P}_{a c t} \cdot \boldsymbol{h}_{1}\right)=\boldsymbol{h}_{1} \frac{1}{R} \frac{\partial}{\partial R} R\left(\boldsymbol{h}_{1} \cdot \boldsymbol{P}_{a c t} \cdot \boldsymbol{h}_{1}\right)+\boldsymbol{k} \frac{1}{R} \frac{\partial}{\partial R} R\left(\boldsymbol{k} \cdot \boldsymbol{P}_{a c t} \cdot \boldsymbol{h}_{1}\right) .
$$

In particular,

$$
\begin{aligned}
\boldsymbol{h}_{1} \cdot \frac{1}{R} \frac{\partial}{\partial R} R\left(\boldsymbol{P}_{a c t} \cdot \boldsymbol{h}_{1}\right) & =\frac{1}{R} \frac{\partial}{\partial R} R\left(\boldsymbol{h}_{1} \cdot \boldsymbol{P}_{a c t} \cdot \boldsymbol{h}_{1}\right) \\
\boldsymbol{k} \cdot \frac{1}{R} \frac{\partial}{\partial R} R\left(\boldsymbol{P}_{a c t} \cdot \boldsymbol{h}_{1}\right) & =\frac{1}{R} \frac{\partial}{\partial R} R\left(\boldsymbol{k} \cdot \boldsymbol{P}_{a c t} \cdot \boldsymbol{h}_{1}\right)
\end{aligned}
$$

We have

$$
\begin{aligned}
& \boldsymbol{d} \times \frac{1}{R} \frac{\partial}{\partial R}\left(R \int_{H_{1}}^{H_{2}} Z\left(\boldsymbol{P}_{a c t} \cdot \boldsymbol{h}_{1}\right) d Z\right) \\
= & \left(\cos \phi \boldsymbol{k}-\sin \phi \boldsymbol{h}_{1}\right) \times\left\{\boldsymbol{h}_{1} \frac{1}{R} \frac{\partial}{\partial R}\left(R \int_{H_{1}}^{H_{2}} Z\left(\boldsymbol{h}_{1} \cdot \boldsymbol{P}_{a c t} \cdot \boldsymbol{h}_{1}\right) d Z\right)\right. \\
& \left.\quad+\boldsymbol{k} \frac{1}{R} \frac{\partial}{\partial R}\left(R \int_{H_{1}}^{H_{2}} Z\left(\boldsymbol{k} \cdot \boldsymbol{P}_{a c t} \cdot \boldsymbol{h}_{1}\right) d Z\right)\right\} \\
= & \boldsymbol{h}_{2} \frac{\cos \phi}{R} \frac{\partial}{\partial R}\left(R \int_{H_{1}}^{H_{2}} Z\left(\boldsymbol{h}_{1} \cdot \boldsymbol{P}_{a c t} \cdot \boldsymbol{h}_{1}\right) d Z\right)+\boldsymbol{h}_{2} \frac{\sin \phi}{R} \frac{\partial}{\partial R}\left(R \int_{H_{1}}^{H_{2}} Z\left(\boldsymbol{k} \cdot \boldsymbol{P}_{a c t} \cdot \boldsymbol{h}_{1}\right) d Z\right) \\
= & \boldsymbol{h}_{2}\left\{\frac{\cos \phi}{R} \frac{\partial}{\partial R}\left(R \int_{H_{1}}^{H_{2}} Z\left(\boldsymbol{h}_{1} \cdot \boldsymbol{P}_{a c t} \cdot \boldsymbol{h}_{1}\right) d Z\right)+\frac{\sin \phi}{R} \frac{\partial}{\partial R}\left(R \int_{H_{1}}^{H_{2}} Z\left(\boldsymbol{k} \cdot \boldsymbol{P}_{a c t} \cdot \boldsymbol{h}_{1}\right) d Z\right)\right\} .
\end{aligned}
$$

The symmetry of the deformation also requires that $\boldsymbol{P}_{\text {act }} \cdot \boldsymbol{h}_{2}$ point along $\boldsymbol{h}_{2}$ :

$$
\left(\boldsymbol{P}_{a c t} \cdot \boldsymbol{h}_{2}\right)=\left(\boldsymbol{h}_{2} \cdot \boldsymbol{P}_{a c t} \cdot \boldsymbol{h}_{2}\right) \boldsymbol{h}_{2} .
$$

Further, the coefficient $\left(\boldsymbol{h}_{2} \cdot \boldsymbol{P}_{a c t} \cdot \boldsymbol{h}_{2}\right)$ is $\Theta$-independent, so the $\Theta$-derivative of $\left(\boldsymbol{P}_{a c t} \cdot \boldsymbol{h}_{2}\right)$ is

$$
\begin{aligned}
\frac{\partial}{\partial \Theta}\left(\boldsymbol{P}_{a c t} \cdot \boldsymbol{h}_{2}\right) & =\frac{\partial}{\partial \Theta}\left(\boldsymbol{h}_{2} \cdot \boldsymbol{P}_{a c t} \cdot \boldsymbol{h}_{2}\right) \boldsymbol{h}_{2} \\
& =\left(\boldsymbol{h}_{2} \cdot \boldsymbol{P}_{a c t} \cdot \boldsymbol{h}_{2}\right) \frac{\partial \boldsymbol{h}_{2}}{\partial \Theta} \\
& =-\left(\boldsymbol{h}_{2} \cdot \boldsymbol{P}_{a c t} \cdot \boldsymbol{h}_{2}\right) \boldsymbol{h}_{1} .
\end{aligned}
$$


The cross-product with $\boldsymbol{d}$ is

$$
\begin{aligned}
\boldsymbol{d} \times \frac{\partial}{\partial \Theta} \int_{H_{1}}^{H_{2}} Z\left(\boldsymbol{P}_{a c t} \cdot \frac{\boldsymbol{h}_{2}}{R}\right) d Z & =\left(\cos \phi \boldsymbol{k}-\sin \phi \boldsymbol{h}_{1}\right) \times\left(-\boldsymbol{h}_{1} \int_{H_{1}}^{H_{2}} Z\left(\boldsymbol{h}_{2} \cdot \boldsymbol{P}_{a c t} \cdot \frac{\boldsymbol{h}_{2}}{R}\right) d Z\right) \\
& =-\boldsymbol{h}_{2} \frac{\cos \phi}{R} \int_{H_{1}}^{H_{2}} Z\left(\boldsymbol{h}_{2} \cdot \boldsymbol{P}_{a c t} \cdot \boldsymbol{h}_{2}\right) d Z .
\end{aligned}
$$

The remaining terms are

$$
\begin{aligned}
\boldsymbol{d} \times \boldsymbol{r},_{\alpha} \int_{H_{1}}^{H_{2}} \beta^{\alpha} d Z & =\boldsymbol{d} \times \frac{\partial \boldsymbol{r}}{\partial R} \int_{H_{1}}^{H_{2}} \beta^{\alpha} d Z+\boldsymbol{d} \times \frac{\partial \boldsymbol{r}}{\partial \Theta} \int_{H_{1}}^{H_{2}} \beta^{\alpha} d Z \\
& =\left(\cos \phi \boldsymbol{k}-\sin \phi \boldsymbol{h}_{1}\right) \times\left\{\left(r^{\prime} \boldsymbol{h}_{1}+\zeta^{\prime} \boldsymbol{k}\right) \int_{H_{1}}^{H_{2}} \beta^{R} d Z+r \boldsymbol{h}_{2} \int_{H_{1}}^{H_{2}} \beta^{\Theta} d Z\right\} \\
& =\left(r^{\prime} \cos \phi \int_{H_{1}}^{H_{2}} \beta^{R} d Z\right) \boldsymbol{h}_{2}+\left(\zeta^{\prime} \sin \phi \int_{H_{1}}^{H_{2}} \beta^{R} d Z\right) \boldsymbol{h}_{2} \\
& -\left(r \cos \phi \int_{H_{1}}^{H_{2}} \beta^{\Theta}\right) \boldsymbol{h}_{1}-\left(r \sin \phi \int_{H_{1}}^{H_{2}} \beta^{\Theta}\right) \boldsymbol{k} .
\end{aligned}
$$

What we have shown is

$$
\begin{aligned}
& \left\{\frac{\cos \phi}{R} \frac{\partial}{\partial R}\left(R \int_{H_{1}}^{H_{2}} Z\left(\boldsymbol{h}_{1} \cdot \boldsymbol{P}_{a c t} \cdot \boldsymbol{h}_{1}\right) d Z\right)+\frac{\sin \phi}{R} \frac{\partial}{\partial R}\left(R \int_{H_{1}}^{H_{2}} Z\left(\boldsymbol{k} \cdot \boldsymbol{P}_{a c t} \cdot \boldsymbol{h}_{1}\right) d Z\right)\right. \\
- & \left.\frac{\cos \phi}{R} \int_{H_{1}}^{H_{2}} Z\left(\boldsymbol{h}_{2} \cdot \boldsymbol{P}_{a c t} \cdot \boldsymbol{h}_{2}\right) d Z\right\} \boldsymbol{h}_{2} \\
= & \left(r^{\prime} \cos \phi+\zeta^{\prime} \sin \phi\right)\left(\int_{H_{1}}^{H_{2}} \beta^{R} d Z\right) \boldsymbol{h}_{2}-r\left(\cos \phi \boldsymbol{h}_{1}-\sin \phi \boldsymbol{k}\right) \int_{H_{1}}^{H_{2}} \beta^{\Theta} d Z .
\end{aligned}
$$

Note that the left-hand side is a scalar multiple of $\boldsymbol{h}_{2}$, and that $\int_{H_{1}}^{H_{2}} \beta^{\Theta} d Z$ on the right-hand side is the coefficient of a linear combination of $\boldsymbol{h}_{1}$ and $\boldsymbol{k}$, both of which are orthogonal to $\boldsymbol{h}_{2}$. Since this holds for each $(R, \Theta)$, we see that $\int_{H_{1}}^{H_{2}} \beta^{\Theta} d Z=0$. Computing the $\boldsymbol{h}_{2}$-projection reveals

$$
\begin{aligned}
& \frac{\cos \phi}{R} \frac{\partial}{\partial R}\left(R \int_{H_{1}}^{H_{2}} Z\left(\boldsymbol{h}_{1} \cdot \boldsymbol{P}_{a c t} \cdot \boldsymbol{h}_{1}\right) d Z\right) \\
& +\frac{\sin \phi}{R} \frac{\partial}{\partial R}\left(R \int_{H_{1}}^{H_{2}} Z\left(\boldsymbol{k} \cdot \boldsymbol{P}_{a c t} \cdot \boldsymbol{h}_{1}\right) d Z\right) \\
& \quad-\frac{\cos \phi}{R} \int_{H_{1}}^{H_{2}} Z\left(\boldsymbol{h}_{2} \cdot \boldsymbol{P}_{a c t} \cdot \boldsymbol{h}_{2}\right) d Z=\left(r^{\prime} \cos \phi+\zeta^{\prime} \sin \phi\right)\left(\int_{H_{1}}^{H_{2}} \beta^{R} d Z\right) .
\end{aligned}
$$


If we multiply by $R$ and divide by $\cos \phi$, we have

$$
\begin{aligned}
\frac{\partial}{\partial R}\left(R \int_{H_{1}}^{H_{2}} Z\left(\boldsymbol{h}_{1} \cdot \boldsymbol{P}_{a c t} \cdot \boldsymbol{h}_{1}\right) d Z\right) & \\
+\tan \phi \frac{\partial}{\partial R}\left(R \int_{H_{1}}^{H_{2}} Z\left(\boldsymbol{k} \cdot \boldsymbol{P}_{a c t} \cdot \boldsymbol{h}_{1}\right) d Z\right) & \\
-\int_{H_{1}}^{H_{2}} Z\left(\boldsymbol{h}_{2} \cdot \boldsymbol{P}_{a c t} \cdot \boldsymbol{h}_{2}\right) d Z & =R\left(r^{\prime}+\zeta^{\prime} \tan \phi\right)\left(\int_{H_{1}}^{H_{2}} \beta^{R} d Z\right) \\
& =R\left(r^{\prime}+r^{\prime} \tan ^{2} \phi\right)\left(\int_{H_{1}}^{H_{2}} \beta^{R} d Z\right) \\
& =R r^{\prime} \sec ^{2} \phi\left(\int_{H_{1}}^{H_{2}} \beta^{R} d Z\right) .
\end{aligned}
$$

In the next section we will find another expression for the integral of $\beta^{R}$, and this equation will become a differential equation in terms of kinematical and constitutively-defined quantities.

\section{Formulation of the problem as a system of ODEs}

Since we have eliminated all differentiation with respect to $\Theta$ in the differential equations, we can view them as a system of ordinary differential equations with independent variable $R$.

Thanks to the discoveries of the last section, Eq. (114) can now be written as

$$
\begin{aligned}
& \frac{1}{R} \frac{d}{d R}\left\{\boldsymbol{h}_{1} R \int_{H_{1}}^{H_{2}}\left(\boldsymbol{h}_{1} \cdot \boldsymbol{P}_{a c t} \cdot \boldsymbol{h}_{1}\right) d Z+\boldsymbol{k} R \int_{H_{1}}^{H_{2}}\left(\boldsymbol{k} \cdot \boldsymbol{P}_{a c t} \cdot \boldsymbol{h}_{1}\right) d Z+R \boldsymbol{d} \int_{H_{1}}^{H_{2}} \beta^{R} d Z\right\} \\
- & \boldsymbol{h}_{1} \int_{H_{1}}^{H_{2}}\left(\boldsymbol{h}_{2} \cdot \boldsymbol{P}_{a c t} \cdot \frac{\boldsymbol{h}_{2}}{R}\right) d Z=\mathbf{0} .
\end{aligned}
$$

The projection of this equation onto $\boldsymbol{k}$ is

$$
\frac{1}{R} \frac{d}{d R}\left\{R \int_{H_{1}}^{H_{2}}\left(\boldsymbol{k} \cdot \boldsymbol{P}_{a c t} \cdot \boldsymbol{h}_{1}\right) d Z+R \cos \phi \int_{H_{1}}^{H_{2}} \beta^{R} d Z\right\}=0,
$$

so that

$$
R \int_{H_{1}}^{H_{2}}\left(\boldsymbol{k} \cdot \boldsymbol{P}_{a c t} \cdot \boldsymbol{h}_{1}\right) d Z+R \cos \phi \int_{H_{1}}^{H_{2}} \beta^{R} d Z=\text { constant. }
$$

Recall that we must assign the value of the stress

$$
\int_{H_{1}}^{H_{2}}\left(\boldsymbol{P}_{a c t} \cdot \boldsymbol{h}_{1}+\beta^{R} \boldsymbol{d}\right) d Z
$$

on the boundary $\left(R=R_{\max }\right)$ of the middle surface. If this stress is zero, then in particular its $\boldsymbol{k}$-projection is zero. This implies that the constant on the right-hand side of Eq. (136) is zero, and

$$
\int_{H_{1}}^{H_{2}} \beta^{R} d Z=-\sec \phi \int_{H_{1}}^{H_{2}}\left(\boldsymbol{k} \cdot \boldsymbol{P}_{a c t} \cdot \boldsymbol{h}_{1}\right) d Z \text {. }
$$


We will see later that the isotropic hyperelastic constitutive relation we choose ensures that $\boldsymbol{P}_{\text {act }} \cdot \boldsymbol{h}_{1}$ points in the direction $\boldsymbol{a}=\cos \phi \boldsymbol{h}_{1}+\sin \phi \boldsymbol{k}$. As a result of the direction of $\boldsymbol{P}_{a c t} \cdot \boldsymbol{h}_{1}$,

$$
\boldsymbol{P}_{a c t} \cdot \boldsymbol{h}_{1}=\left(\boldsymbol{a} \cdot \boldsymbol{P}_{a c t} \cdot \boldsymbol{h}_{1}\right) \boldsymbol{a},
$$

so $\left(\boldsymbol{h}_{1} \cdot \boldsymbol{P}_{\text {act }} \cdot \boldsymbol{h}_{1}\right)$ and $\left(\boldsymbol{k} \cdot \boldsymbol{P}_{\text {act }} \cdot \boldsymbol{h}_{1}\right)$ satisfy

$$
\begin{aligned}
\boldsymbol{k} \cdot \boldsymbol{P}_{a c t} \cdot \boldsymbol{h}_{1} & =\left(\boldsymbol{a} \cdot \boldsymbol{P}_{a c t} \cdot \boldsymbol{h}_{1}\right) \boldsymbol{k} \cdot \boldsymbol{a}=\sin \phi\left(\boldsymbol{a} \cdot \boldsymbol{P}_{a c t} \cdot \boldsymbol{h}_{1}\right), \\
\boldsymbol{h}_{1} \cdot \boldsymbol{P}_{a c t} \cdot \boldsymbol{h}_{1} & =\left(\boldsymbol{a} \cdot \boldsymbol{P}_{a c t} \cdot \boldsymbol{h}_{1}\right) \boldsymbol{h}_{1} \cdot \boldsymbol{a}=\cos \phi\left(\boldsymbol{a} \cdot \boldsymbol{P}_{a c t} \cdot \boldsymbol{h}_{1}\right), \\
\text { and } \quad\left(\boldsymbol{k} \cdot \boldsymbol{P}_{a c t} \cdot \boldsymbol{h}_{1}\right) & =\tan \phi\left(\boldsymbol{h}_{1} \cdot \boldsymbol{P}_{a c t} \cdot \boldsymbol{h}_{1}\right) .
\end{aligned}
$$

and

$$
\int_{H_{1}}^{H_{2}} \beta^{R} d Z=-\sec \phi \tan \phi \int_{H_{1}}^{H_{2}}\left(\boldsymbol{h}_{1} \cdot \boldsymbol{P}_{a c t} \cdot \boldsymbol{h}_{1}\right) d Z \text {. }
$$

The $\boldsymbol{h}_{1}$-projection of Eq. (134) becomes

$$
\begin{aligned}
\frac{1}{R} \frac{d}{d R}\left\{R \int_{H_{1}}^{H_{2}}\left(\boldsymbol{h}_{1} \cdot \boldsymbol{P}_{a c t} \cdot \boldsymbol{h}_{1}\right) d Z-R \sin \phi \int_{H_{1}}^{H_{2}} \beta^{R} d Z\right\} & =\int_{H_{1}}^{H_{2}}\left(\boldsymbol{h}_{2} \cdot \boldsymbol{P}_{a c t} \cdot \frac{\boldsymbol{h}_{2}}{R}\right) d Z \\
\frac{1}{R} \frac{d}{d R}\left\{R \int_{H_{1}}^{H_{2}}\left(\boldsymbol{h}_{1} \cdot \boldsymbol{P}_{a c t} \cdot \boldsymbol{h}_{1}\right) d Z+R \tan ^{2} \phi \int_{H_{1}}^{H_{2}}\left(\boldsymbol{h}_{1} \cdot \boldsymbol{P}_{a c t} \cdot \boldsymbol{h}_{1}\right) d Z\right\} & =\int_{H_{1}}^{H_{2}}\left(\boldsymbol{h}_{2} \cdot \boldsymbol{P}_{a c t} \cdot \frac{\boldsymbol{h}_{2}}{R}\right) d Z \\
\frac{d}{d R}\left\{R \sec ^{2} \phi \int_{H_{1}}^{H_{2}}\left(\boldsymbol{h}_{1} \cdot \boldsymbol{P}_{a c t} \cdot \boldsymbol{h}_{1}\right) d Z\right\} & =\int_{H_{1}}^{H_{2}}\left(\boldsymbol{h}_{2} \cdot \boldsymbol{P}_{a c t} \cdot \boldsymbol{h}_{2}\right) d Z .
\end{aligned}
$$

We have one more scalar ordinary differential equation from the previous section:

$$
\begin{aligned}
\frac{d}{d R}\left(R \int_{H_{1}}^{H_{2}} Z\left(\boldsymbol{h}_{1} \cdot \boldsymbol{P}_{a c t} \cdot \boldsymbol{h}_{1}\right) d Z\right) & \\
+\tan \phi \frac{d}{d R}\left(R \int_{H_{1}}^{H_{2}} Z\left(\boldsymbol{k} \cdot \boldsymbol{P}_{a c t} \cdot \boldsymbol{h}_{1}\right) d Z\right) & \\
\quad-\int_{H_{1}}^{H_{2}} Z\left(\boldsymbol{h}_{2} \cdot \boldsymbol{P}_{a c t} \cdot \boldsymbol{h}_{2}\right) d Z & =R r^{\prime} \sec ^{2} \phi\left(\int_{H_{1}}^{H_{2}} \beta^{R} d Z\right) \\
& =-R r^{\prime} \tan \phi \sec ^{3} \phi \int_{H_{1}}^{H_{2}}\left(\boldsymbol{h}_{1} \cdot \boldsymbol{P}_{a c t} \cdot \boldsymbol{h}_{1}\right) d Z,
\end{aligned}
$$

where we have used Eq. (143) to re-write the right-hand side. Note that

$$
\begin{aligned}
& \tan \phi \frac{d}{d R}\left(R \int_{H_{1}}^{H_{2}} Z\left(\boldsymbol{k} \cdot \boldsymbol{P}_{a c t} \cdot \boldsymbol{h}_{1}\right) d Z\right) \\
= & \tan \phi \frac{d}{d R}\left(R \tan \phi \int_{H_{1}}^{H_{2}} Z\left(\boldsymbol{h}_{1} \cdot \boldsymbol{P}_{a c t} \cdot \boldsymbol{h}_{1}\right) d Z\right) \\
= & \phi^{\prime} \tan \phi \sec ^{2} \phi R \int_{H_{1}}^{H_{2}} Z\left(\boldsymbol{h}_{1} \cdot \boldsymbol{P}_{a c t} \cdot \boldsymbol{h}_{1}\right) d Z+\tan ^{2} \phi \frac{d}{d R}\left(R \int_{H_{1}}^{H_{2}} Z\left(\boldsymbol{h}_{1} \cdot \boldsymbol{P}_{a c t} \cdot \boldsymbol{h}_{1}\right) d Z\right) .
\end{aligned}
$$


Eq. (145) can be re-written as

$$
\begin{aligned}
& \left(1+\tan ^{2} \phi\right) \frac{d}{d R}\left(R \int_{H_{1}}^{H_{2}} Z\left(\boldsymbol{h}_{1} \cdot \boldsymbol{P}_{a c t} \cdot \boldsymbol{h}_{1}\right) d Z\right)+\phi^{\prime} \tan \phi \sec ^{2} \phi R \int_{H_{1}}^{H_{2}} Z\left(\boldsymbol{h}_{1} \cdot \boldsymbol{P}_{a c t} \cdot \boldsymbol{h}_{1}\right) d Z \\
= & \int_{H_{1}}^{H_{2}} Z\left(\boldsymbol{h}_{2} \cdot \boldsymbol{P}_{a c t} \cdot \boldsymbol{h}_{2}\right) d Z-R r^{\prime} \tan \phi \sec ^{3} \phi \int_{H_{1}}^{H_{2}}\left(\boldsymbol{h}_{1} \cdot \boldsymbol{P}_{a c t} \cdot \boldsymbol{h}_{1}\right) d Z .
\end{aligned}
$$

Since $1+\tan ^{2} \phi=\sec ^{2} \phi=(\cos \phi)^{-2}$, we can re-write this as

$$
\begin{aligned}
\frac{d}{d R}\left(R \int_{H_{1}}^{H_{2}} Z\left(\boldsymbol{h}_{1} \cdot \boldsymbol{P}_{a c t} \cdot \boldsymbol{h}_{1}\right) d Z\right) & =\cos ^{2} \phi \int_{H_{1}}^{H_{2}} Z\left(\boldsymbol{h}_{2} \cdot \boldsymbol{P}_{a c t} \cdot \boldsymbol{h}_{2}\right) d Z \\
& -\left(R \phi^{\prime}+R r^{\prime} \sec \phi\right) \tan \phi \int_{H_{1}}^{H_{2}} Z\left(\boldsymbol{h}_{1} \cdot \boldsymbol{P}_{a c t} \cdot \boldsymbol{h}_{1}\right) d Z .
\end{aligned}
$$

In summary, the balance laws for linear momentum and angular momentum for the plate are

$$
\begin{aligned}
\frac{d}{d R}\left(R \sec ^{2} \phi \int_{H_{1}}^{H_{2}}\left(\boldsymbol{h}_{1} \cdot \boldsymbol{P}_{a c t} \cdot \boldsymbol{h}_{1}\right) d Z\right) & =\int_{H_{1}}^{H_{2}}\left(\boldsymbol{h}_{2} \cdot \boldsymbol{P}_{a c t} \cdot \boldsymbol{h}_{2}\right) d Z \\
\frac{d}{d R}\left(R \int_{H_{1}}^{H_{2}} Z\left(\boldsymbol{h}_{1} \cdot \boldsymbol{P}_{a c t} \cdot \boldsymbol{h}_{1}\right) d Z\right) & =\cos ^{2} \phi \int_{H_{1}}^{H_{2}} Z\left(\boldsymbol{h}_{2} \cdot \boldsymbol{P}_{a c t} \cdot \boldsymbol{h}_{2}\right) d Z \\
& -\left(R \phi^{\prime}+R r^{\prime} \sec \phi\right) \tan \phi \int_{H_{1}}^{H_{2}} Z\left(\boldsymbol{h}_{1} \cdot \boldsymbol{P}_{a c t} \cdot \boldsymbol{h}_{1}\right) d Z .
\end{aligned}
$$

We have already ensured that

$$
\int_{H_{1}}^{H_{2}}\left(\boldsymbol{k} \cdot \boldsymbol{P}_{a c t} \cdot \boldsymbol{h}_{1}\right) d Z+\cos \phi \int_{H_{1}}^{H_{2}} \beta^{R} d Z=\boldsymbol{k} \cdot\left\{\int_{H_{1}}^{H_{2}}\left(\boldsymbol{P}_{a c t} \cdot \boldsymbol{h}_{1}\right) d Z+\boldsymbol{d} \int_{H_{1}}^{H_{2}} \beta^{R} d Z\right\}
$$

is identically zero. One of the boundary conditions is that the analogous $\boldsymbol{h}_{1}$-projection is zero at the boundary of the middle surface:

$$
\begin{aligned}
& \boldsymbol{h}_{1} \cdot\left\{\int_{H_{1}}^{H_{2}}\left(\boldsymbol{P}_{a c t} \cdot \boldsymbol{h}_{1}\right) d Z+\boldsymbol{d} \int_{H_{1}}^{H_{2}} \beta^{R} d Z\right\} \\
= & \int_{H_{1}}^{H_{2}}\left(\boldsymbol{h}_{1} \cdot \boldsymbol{P}_{a c t} \cdot \boldsymbol{h}_{1}\right) d Z-\sin \phi \int_{H_{1}}^{H_{2}} \beta^{R} d Z \\
= & \int_{H_{1}}^{H_{2}}\left(\boldsymbol{h}_{1} \cdot \boldsymbol{P}_{a c t} \cdot \boldsymbol{h}_{1}\right) d Z+\sin \phi \sec \phi \int_{H_{1}}^{H_{2}}\left(\boldsymbol{k} \cdot \boldsymbol{P}_{a c t} \cdot \boldsymbol{h}_{1}\right) d Z \\
= & \left(1+\tan ^{2} \phi\right) \int_{H_{1}}^{H_{2}}\left(\boldsymbol{h}_{1} \cdot \boldsymbol{P}_{a c t} \cdot \boldsymbol{h}_{1}\right) d Z \\
= & \sec ^{2} \phi \int_{H_{1}}^{H_{2}}\left(\boldsymbol{h}_{1} \cdot \boldsymbol{P}_{a c t} \cdot \boldsymbol{h}_{1}\right) d Z .
\end{aligned}
$$


Hence the boundary condition for stress is

$$
\left.\sec ^{2} \phi \int_{H_{1}}^{H_{2}}\left(\boldsymbol{h}_{1} \cdot \boldsymbol{P}_{a c t} \cdot \boldsymbol{h}_{1}\right) d Z\right|_{R \in\left\{R_{\min }, R_{\max }\right\}}=0,
$$

where we consider $R_{\max }$ in every case and $R_{\min }$ only in the case of an annulus.

The boundary condition for moment is

$$
\left.\int_{H_{1}}^{H_{2}} Z\left(\boldsymbol{P}_{a c t} \cdot \boldsymbol{h}_{1}\right) d Z\right|_{R \in\left\{R_{\min }, R_{\max }\right\}}=\mathbf{0} .
$$

We will find a scalar equation that ensures that the $\boldsymbol{h}_{1^{-}}$and $\boldsymbol{k}$-projections are both zero.

\section{Applying the Constitutive Relation}

We consider an isotropic hyperelastic constitutive relation derived from a strain energy density of neo-Hookean type:

$$
W\left(\lambda_{1}, \lambda_{2}, \lambda_{3}\right)=\mu\left(\frac{1}{2}\left(\lambda_{1}^{2}+\lambda_{2}^{2}+\lambda_{3}^{3}-3\right)-\ln J\right)+\lambda(J-1-\ln J),
$$

where $\lambda_{i}, i=1,2,3$, are the principal stretches of the deformation, and $J=\lambda_{1} \lambda_{2} \lambda_{3}$. The constants $\lambda$ and $\mu$ correspond to the Lamé moduli of linear elasticity.

\subsection{Without Growth}

We will first consider the case without growth and establish the form of Cauchy stress tensor as a function of the deformation gradient $\boldsymbol{F}$.

One of the polar decompositions of the deformation gradient is $\boldsymbol{F}=\boldsymbol{R} \cdot \boldsymbol{U}$, where $\boldsymbol{R}$ is a proper rotation and $\boldsymbol{U}=\sqrt{\boldsymbol{F}^{T} \boldsymbol{F}}$ is a symmetric positive-definite tensor called the right stretch tensor. Since the strain-energy density $W\left(\lambda_{1}, \lambda_{2}, \lambda_{3}\right)$ is an isotropic function of the deformation gradient, it is equal to some function $\widetilde{W}(\boldsymbol{U})$. We compute the Biot stress or the Jaumann stress

$$
\boldsymbol{T}^{(1)}=\frac{\partial \widetilde{W}}{\partial \boldsymbol{U}}=\sum_{i=1}^{3} \frac{\partial W}{\partial \lambda_{i}} \boldsymbol{u}^{(i)} \otimes \boldsymbol{u}^{(i)},
$$

where the $\boldsymbol{u}^{(i)}$ are the unit eigenvectors of $\boldsymbol{U}$.

In isotropic hyperelasticity without local constraints, the Cauchy stress and Biot/Jaumann stress are related by

$$
\begin{aligned}
J \boldsymbol{R}^{T} \cdot \boldsymbol{T} \cdot \boldsymbol{R} & =\boldsymbol{U} \cdot \boldsymbol{T}^{(1)} \\
& =\sum_{i=1}^{3} \frac{\partial W}{\partial \lambda_{i}}\left(\boldsymbol{U} \cdot \boldsymbol{u}^{(i)}\right) \otimes \boldsymbol{u}^{(i)} \\
& =\sum_{i=1}^{3} \lambda_{i} \frac{\partial W}{\partial \lambda_{i}} \boldsymbol{u}^{(i)} \otimes \boldsymbol{u}^{(i)}
\end{aligned}
$$


See section 4.3 of [13].

The principal components of the active Cauchy stress (the constitutively-defined portion of the Cauchy stress) are then

$$
\left(\boldsymbol{T}_{a c t}\right)_{i}=\frac{\lambda_{i}}{J} \frac{\partial W}{\partial \lambda_{i}}=\frac{1}{J}\left(\mu\left(\lambda_{i}^{2}-1\right)+\lambda(J-1)\right) .
$$

\subsection{With Growth}

When growth is included via multiplicative decomposition of the deformation gradient $\boldsymbol{F}=$ $\boldsymbol{A} \cdot \boldsymbol{G}$, the (active) Cauchy stress is a function of the elastic portion $\boldsymbol{A}$ alone. However, we can import the results found above. Eq. (158) still holds, but the eigenvalues are eigenvalues of $\boldsymbol{A}$ (actually, square roots of eigenvalues of $\boldsymbol{A}^{T} \cdot \boldsymbol{A}$ ), and the principal directions are the eigenvectors of $\boldsymbol{A}^{T} \cdot \boldsymbol{A}$. By Eq. (104), the full active Cauchy stress tensor is

$$
\boldsymbol{T}_{a c t}=\frac{1}{J}\left(\mu\left(\lambda_{1}^{2} \boldsymbol{a} \otimes \boldsymbol{a}+\lambda_{2}^{2} \boldsymbol{h}_{2} \otimes \boldsymbol{h}_{2}+\lambda_{3}^{2} \boldsymbol{d} \otimes \boldsymbol{d}\right)+(\lambda J-(\lambda+\mu)) \boldsymbol{I}\right),
$$

where the $\lambda_{i}$ are the "pseudo-stretches"

$$
\lambda_{1}=\frac{r^{\prime} \sec \phi-Z \phi^{\prime}}{\gamma_{1}}, \quad \lambda_{2}=\frac{r-Z \sin \phi}{\gamma_{2} R}, \quad \lambda_{3}=1,
$$

and $J=\operatorname{det} \boldsymbol{A}=\lambda_{1} \lambda_{2} \lambda_{3}$.

To differential equations Eqs. (149) and (150) are written in terms of $\boldsymbol{P}_{a c t}=(\operatorname{det} \boldsymbol{F}) \boldsymbol{T}_{a c t}$. $\boldsymbol{F}^{-T}$, but we need only certain projections of this tensor. Note that

$$
\begin{aligned}
\boldsymbol{F}^{-T} \cdot \boldsymbol{h}_{1} & =\left(r^{\prime} \sec \phi-Z \phi^{\prime}\right)^{-1} \boldsymbol{a}, \\
\boldsymbol{F}^{-T} \cdot \boldsymbol{h}_{2} & =\left(\frac{r-Z \sin \phi}{R}\right)^{-1} \boldsymbol{h}_{2} .
\end{aligned}
$$

We have

$$
\begin{aligned}
\boldsymbol{P}_{a c t} \cdot \boldsymbol{h}_{1} & =(\operatorname{det} \boldsymbol{F}) \boldsymbol{T}_{a c t} \cdot \boldsymbol{F}^{-T} \cdot \boldsymbol{h}_{1} \\
& =J \gamma_{1} \gamma_{2} \boldsymbol{T}_{a c t} \cdot\left(r^{\prime} \sec \phi-Z \phi^{\prime}\right)^{-1} \boldsymbol{a} \\
& =\gamma_{1} \gamma_{2}\left(\frac{\mu}{\gamma_{1}^{2}}\left(r^{\prime} \sec \phi-Z \phi^{\prime}\right)+\frac{\lambda}{\gamma_{1} \gamma_{2}}\left(r^{\prime} \sec \phi-Z \phi^{\prime}\right)-\frac{\lambda+\mu}{r^{\prime} \sec \phi-Z \phi^{\prime}}\right) \boldsymbol{a},(16
\end{aligned}
$$

where

$$
\operatorname{det} \boldsymbol{F}=(\operatorname{det} \boldsymbol{A})(\operatorname{det} \boldsymbol{G})=(J)\left(\gamma_{1} \gamma_{2}\right) .
$$

A similar calculation shows that

$$
\boldsymbol{P}_{a c t} \cdot \boldsymbol{h}_{2}=\gamma_{1} \gamma_{2}\left(\frac{\mu}{\gamma_{2}^{2}}\left(\frac{r-Z \sin \phi}{R}\right)+\frac{\lambda}{\gamma_{1} \gamma_{2} \gamma_{3}}\left(\frac{r-Z \sin \phi}{R}\right)-\frac{\lambda+\mu}{(r-Z \sin \phi) / R}\right) \boldsymbol{h}_{2} \text {. }
$$

The projections pertinent for the differential equations are

$$
\begin{aligned}
& \boldsymbol{h}_{1} \cdot \boldsymbol{P}_{a c t} \cdot \boldsymbol{h}_{1}=\cos \phi\left(\frac{\mu \gamma_{2}}{\gamma_{1}}\left(r^{\prime} \sec \phi-\phi^{\prime} Z\right)+\lambda\left(\frac{r-Z \sin \phi}{R}\right)-\frac{(\lambda+\mu) \gamma_{1} \gamma_{2}}{r^{\prime} \sec \phi-Z \phi^{\prime}}\right) \\
& \boldsymbol{h}_{2} \cdot \boldsymbol{P}_{a c t} \cdot \boldsymbol{h}_{2}=\frac{\mu \gamma_{1}}{\gamma_{2}}\left(\frac{r-Z \sin \phi}{R}\right)+\lambda\left(r^{\prime} \sec \phi-Z \phi^{\prime}\right)-\frac{(\lambda+\mu) \gamma_{1} \gamma_{2}}{(r-Z \sin \phi) / R}
\end{aligned}
$$


We also assume that the middle surface occupies the true middle of the plate, so that $H_{1}=-H$ and $H_{2}=H$ for some $H>0$. The $Z$-integrals then have the forms

$$
\begin{aligned}
\int_{-H}^{H}\left(\boldsymbol{h}_{1} \cdot \boldsymbol{P}_{a c t} \cdot \boldsymbol{h}_{1}\right) d Z= & \cos \phi\left\{2 H\left(\frac{\mu \gamma_{2}}{\gamma_{1}} r^{\prime} \sec \phi+\lambda \frac{r}{R}\right)-\frac{2(\lambda+\mu) \gamma_{1} \gamma_{2}}{\phi^{\prime}} \operatorname{arctanh}\left(\frac{H \phi^{\prime}}{r^{\prime} \sec \phi}\right)\right\} \\
\int_{-H}^{H}\left(\boldsymbol{h}_{2} \cdot \boldsymbol{P}_{a c t} \cdot \boldsymbol{h}_{2}\right) d Z= & 2 H\left(\frac{\mu \gamma_{1}}{\gamma_{2}} \frac{r}{R}+\lambda r^{\prime} \sec \phi\right)-\frac{2(\lambda+\mu) \gamma_{1} \gamma_{2} R}{\sin \phi} \operatorname{arctanh}\left(\frac{H \sin \phi}{r}\right)(169) \\
\int_{-H}^{H} Z\left(\boldsymbol{h}_{1} \cdot \boldsymbol{P}_{a c t} \cdot \boldsymbol{h}_{1}\right) d Z= & -\cos \phi\left\{\frac{2}{3} H^{3}\left(\frac{\mu \gamma_{2}}{\gamma_{1}} \phi^{\prime}+\lambda \frac{\sin \phi}{R}\right)\right. \\
& \left.+\frac{2(\lambda+\mu) \gamma_{1} \gamma_{2}}{\left(\phi^{\prime}\right)^{2}}\left(r^{\prime} \sec \phi \operatorname{arctanh}\left(\frac{H \phi^{\prime}}{r^{\prime} \sec \phi}\right)-H \phi^{\prime}\right)\right\} \\
\int_{-H}^{H} Z\left(\boldsymbol{h}_{2} \cdot \boldsymbol{P}_{a c t} \cdot \boldsymbol{h}_{2}\right) d Z= & -\frac{2}{3} H^{3}\left(\frac{\mu \gamma_{1}}{\gamma_{2}} \frac{\sin \phi}{R}+\lambda \phi^{\prime}\right) \\
& -\frac{2(\lambda+\mu) \gamma_{1} \gamma_{2} R}{\sin ^{2} \phi}\left(r \operatorname{arctanh}\left(\frac{H \sin \phi}{r}\right)-H \sin \phi\right)
\end{aligned}
$$

We get a further simplification if we divide by $\mu$ :

$$
\begin{aligned}
\frac{1}{\mu} \int_{-H}^{H}\left(\boldsymbol{h}_{1} \cdot \boldsymbol{P}_{a c t} \cdot \boldsymbol{h}_{1}\right) d Z= & \cos \phi\left\{2 H\left(\frac{\gamma_{2}}{\gamma_{1}} r^{\prime} \sec \phi+\kappa \frac{r}{R}\right)-\frac{2(1+\kappa) \gamma_{1} \gamma_{2}}{\phi^{\prime}} \operatorname{arctanh}\left(\frac{H \phi^{\prime}}{r^{\prime} \sec \phi}\right)\right\} \\
\frac{1}{\mu} \int_{-H}^{H}\left(\boldsymbol{h}_{2} \cdot \boldsymbol{P}_{a c t} \cdot \boldsymbol{h}_{2}\right) d Z= & 2 H\left(\frac{\gamma_{1}}{\gamma_{2}} \frac{r}{R}+\kappa r^{\prime} \sec \phi\right)-\frac{2(1+\kappa) \gamma_{1} \gamma_{2} R}{\sin \phi} \operatorname{arctanh}\left(\frac{H \sin \phi}{r}\right)(173) \\
\frac{1}{\mu} \int_{-H}^{H} Z\left(\boldsymbol{h}_{1} \cdot \boldsymbol{P}_{a c t} \cdot \boldsymbol{h}_{1}\right) d Z= & -\cos \phi\left\{\frac{2}{3} H^{3}\left(\frac{\gamma_{2}}{\gamma_{1}} \phi^{\prime}+\kappa \frac{\sin \phi}{R}\right)\right. \\
& \left.+\frac{2(1+\kappa) \gamma_{1} \gamma_{2}}{\left(\phi^{\prime}\right)^{2}}\left(r^{\prime} \sec \phi \operatorname{arctanh}\left(\frac{H \phi^{\prime}}{r^{\prime} \sec \phi}\right)-H \phi^{\prime}\right)\right\} \\
\frac{1}{\mu} \int_{-H}^{H} Z\left(\boldsymbol{h}_{2} \cdot \boldsymbol{P}_{a c t} \cdot \boldsymbol{h}_{2}\right) d Z= & -\frac{2}{3} H^{3}\left(\frac{\gamma_{1}}{\gamma_{2}} \frac{\sin \phi}{R}+\kappa \phi^{\prime}\right) \\
& -\frac{2(1+\kappa) \gamma_{1} \gamma_{2} R}{\sin ^{2} \phi}\left(r \operatorname{arctanh}\left(\frac{H \sin \phi}{r}\right)-H \sin \phi\right),
\end{aligned}
$$

where $\kappa=\lambda / \mu$. The differential equations have the same form if each stress and moment is replaced by the same quantity divided by $\mu$.

\section{$8 \quad$ Stability of the Flat Plate}

\subsection{The Flat Plate}

If the plate is un-buckled, then there is no moment, $\boldsymbol{P}_{a c t} \cdot \boldsymbol{h}_{1}$ has no $Z$-dependence, and the radial Piola-Kirchhoff stress is radial only, i.e. it has no vertical component. In the case of 
constant $\gamma_{1}$ and $\gamma_{2}$, the equations for the un-buckled plate can be converted into a pair of autonomous ordinary differential equations, as detailed in [3]. If we set

$$
\begin{aligned}
R & =R_{\max } e^{s-1} \\
\tau & =\frac{r}{R}, \\
N & =\frac{\gamma_{2}}{\gamma_{1}} r^{\prime}+\kappa \frac{r}{R}-\frac{(1+\kappa) \gamma_{1} \gamma_{2}}{r^{\prime}} .
\end{aligned}
$$

When $\tau$ and $N$ are viewed as functions of the independent variable $s$, they satisfy

$$
\begin{aligned}
\frac{d \tau}{d s} & =\frac{\gamma_{1}}{2 \gamma_{2}}\left(N-\kappa \tau+\sqrt{(N-\kappa \tau)^{2}+4(1+\kappa) \gamma_{2}^{2}}\right)-\tau \\
\frac{d N}{d s} & =\frac{\gamma_{1}}{\gamma_{2}} \tau+\frac{\kappa \gamma_{1}}{2 \gamma_{2}}\left(N-\kappa \tau+\sqrt{(N-\kappa \tau)^{2}+4(1+\kappa) \gamma_{2}^{2}}\right)-\frac{(1+\kappa) \gamma_{1} \gamma_{2}}{\tau}-N
\end{aligned}
$$

We consider an annulus that has zero radial stress at its inner and outer faces. We can find numerically an initial value for $\tau$ such that the boundary conditions $n\left(s_{0}\right)=0$ and $n\left(s_{1}\right)=0$. The functions $n(s)$ and $\tau(s)$ correspond to functions $N(R)$ and $T(R)$ via

$$
N(R)=n\left(1+\ln \left(\frac{R}{R_{\max }}\right)\right), \quad T(R)=\tau\left(1+\ln \left(\frac{R}{R_{\max }}\right)\right) .
$$

This correspondence also gives us a function $r(R)$ :

$$
r(R)=R T(R)=R \tau\left(1+\ln \left(\frac{R}{R_{\max }}\right)\right),
$$

which also provides a function $r^{\prime}(R)$.

\subsection{Differential Equations for Bifurcation}

Finding numerical solutions of the boundary-value problem for the buckled plate has proved a daunting task. To demonstrate the existence of buckled solutions, we consider perturbation about the flat configuration.

Eqs. (149) and (150) for the buckled plate, i.e. with stresses and moments given by Eqs. (168)-(171), can be expressed in the form

$$
\underbrace{\left(\begin{array}{cccc}
1 & 0 & 0 & 0 \\
0 & 1 & 0 & 0 \\
0 & 0 & a_{33} & a_{34} \\
0 & 0 & a_{43} & a_{44}
\end{array}\right)}_{A\left(R, r, \phi, r^{\prime}, \phi^{\prime}\right)} \frac{d}{d R}\left(\begin{array}{c}
r \\
\phi \\
r^{\prime} \\
\phi^{\prime}
\end{array}\right)=\underbrace{\left(\begin{array}{c}
r^{\prime} \\
\phi^{\prime} \\
f_{3} \\
f_{4}
\end{array}\right)}_{\mathbf{f}\left(R, r, \phi, r^{\prime}, \phi^{\prime}\right)},
$$

with

$$
a_{33}=2 H R\left(\frac{\gamma_{2} \sec ^{2} \phi}{\gamma_{1}}+\frac{\gamma_{1} \gamma_{2}(1+\kappa)}{\left(r^{\prime}\right)^{2}-H^{2}\left(\phi^{\prime}\right)^{2} \cos ^{2} \phi}\right)
$$




$$
\begin{aligned}
& a_{34}=2 R\left\{\frac{\gamma_{1} \gamma_{2}(1+\kappa) \sec \phi}{\left(\phi^{\prime}\right)^{2}} \operatorname{arctanh}\left(\frac{H \phi^{\prime} \cos \phi}{r^{\prime}}\right)\right. \\
& \left.-\frac{H \gamma_{1} \gamma_{2}(1+\kappa)}{r^{\prime} \phi^{\prime}\left(1-\frac{H^{2}\left(\phi^{\prime}\right)^{2} \cos ^{2} \phi}{\left(r^{\prime}\right)^{2}}\right)}\right\} \\
& a_{43}=2 R\left\{-\frac{\gamma_{1} \gamma_{2}(1+\kappa)}{\left(\phi^{\prime}\right)^{2}} \operatorname{arctanh}\left(\frac{H \phi^{\prime} \cos \phi}{r^{\prime}}\right)\right. \\
& \left.+\frac{H \gamma_{1} \gamma_{2}(1+\kappa) \cos \phi}{r^{\prime} \phi^{\prime}\left(1-\frac{H^{2}\left(\phi^{\prime}\right)^{2} \cos ^{2} \phi}{\left(r^{\prime}\right)^{2}}\right)}\right\} \\
& a_{44}=2 R\left\{-\frac{H^{3} \gamma_{2} \cos \phi}{3 \gamma_{1}}+\frac{H \gamma_{1} \gamma_{2}(1+\kappa) \cos \phi}{\left(\phi^{\prime}\right)^{2}}\right. \\
& +\frac{2 \gamma_{1} \gamma_{2}(1+\kappa) \cos \phi\left(\operatorname{arctanh}\left(\frac{H \phi^{\prime} \cos \phi}{r^{\prime}}\right) r^{\prime} \sec \phi-H \phi^{\prime}\right)}{\left(\phi^{\prime}\right)^{3}} \\
& \left.-\frac{H \gamma_{1} \gamma_{2}(1+\kappa) \cos \phi}{\left(\phi^{\prime}\right)^{2}\left(1-\frac{H^{2}\left(\phi^{\prime}\right)^{2} \cos ^{2} \phi}{\left(r^{\prime}\right)^{2}}\right)}\right\} \\
& f_{3}=2 R \gamma_{1} \gamma_{2}(1+\kappa) \operatorname{arctanh}\left(\frac{H \sin \phi}{r}\right) \csc \phi-\frac{2 H \kappa r \sec \phi}{R} \\
& +2 H \kappa r^{\prime} \sec \phi-2 H\left(\frac{\gamma_{1} r}{R \gamma_{2}}+\kappa r^{\prime} \sec \phi\right) \\
& +\sec \phi\left(2 H\left(\frac{\kappa r}{R}+\frac{\gamma_{2} r^{\prime} \sec \phi}{\gamma_{1}}\right)-\frac{2 \gamma_{1} \gamma_{2}(1+\kappa) \operatorname{arctanh}\left(\frac{H \phi^{\prime} \cos \phi}{r^{\prime}}\right)}{\phi}\right) \\
& +\frac{2 H R \gamma_{2} r^{\prime} \phi^{\prime} \sec ^{2} \phi \tan \phi}{\gamma_{1}} \\
& +R \sec \phi \tan \phi\left(2 H\left(\frac{\kappa r}{R}+\frac{\gamma_{2} r^{\prime} \sec \phi}{\gamma_{1}}\right)-\frac{2 \gamma_{1} \gamma_{2}(1+\kappa) \operatorname{arctanh}\left(\frac{H \phi^{\prime} \cos \phi}{r^{\prime}}\right)}{\phi^{\prime}}\right) \phi^{\prime} \\
& +\frac{2 H R \gamma_{1} \gamma_{2}(1+\kappa) \phi^{\prime} \tan \phi}{r^{\prime}\left(1-\frac{H^{2}\left(\phi^{\prime}\right)^{2} \cos ^{2} \phi}{\left(r^{\prime}\right)^{2}}\right)}
\end{aligned}
$$




$$
\begin{aligned}
f_{4} & =\frac{2 H^{3} \kappa \cos \phi \sin \phi}{3 R} \\
& +R r^{\prime} \tan \phi\left(2 H\left(\frac{\kappa r}{R}+\frac{\gamma_{2} r^{\prime} \sec \phi}{\gamma_{1}}\right)-\frac{2 \gamma_{1} \gamma_{2}(1+\kappa) \operatorname{arctanh}\left(\frac{H \phi^{\prime} \cos \phi}{r^{\prime}}\right)}{\phi^{\prime}}\right) \\
& -\frac{2 R \gamma_{1} \gamma_{2}(1+\kappa) \operatorname{arctanh}\left(\frac{H \phi^{\prime} \cos \phi}{r^{\prime}}\right) r^{\prime} \tan \phi}{\phi^{\prime}} \\
& -\frac{2}{3} H^{3} \kappa \phi^{\prime} \cos ^{2} \phi+\frac{2 H R \gamma_{1} \gamma_{2}(1+\kappa) \sin \phi}{1-\frac{H^{2}\left(\phi^{\prime}\right)^{2} \cos ^{2} \phi}{\left(r^{\prime}\right)^{2}}} \\
& -\cos \phi\left\{\frac{2 \gamma_{1} \gamma_{2}(1+\kappa)\left(\operatorname{arctanh}^{\prime}\left(\frac{H \phi^{\prime} \cos \phi}{r^{\prime}}\right) r^{\prime} \sec \phi-H \phi^{\prime}\right)}{\left(\phi^{\prime}\right)^{2}}\right. \\
& \left.+\frac{2}{3} H^{3}\left(\frac{\kappa \sin \phi}{R}+\frac{\gamma_{2} \phi^{\prime}}{\gamma_{1}}\right)\right\} \\
- & \cos \phi\left\{-2 R \gamma_{1} \gamma_{2}(1+\kappa) \csc ^{2} \phi\left(r \operatorname{arctanh}\left(\frac{H \sin \phi}{r}\right)-H \sin \phi\right)\right. \\
- & \left.\frac{2}{3} H^{3}\left(\frac{\gamma_{1} \sin \phi}{R \gamma_{2}}+\kappa \phi^{\prime}\right)\right\}
\end{aligned}
$$

The matrix $A$ is invertible symbolically, so the equations can be expressed in quasilinear form:

$$
\frac{d}{d R}\left(\begin{array}{c}
r \\
\phi \\
r^{\prime} \\
\phi^{\prime}
\end{array}\right)=A^{-1} \mathbf{f}
$$

We consider expressions $r=r_{0}+\epsilon r_{1}$ and $\phi=\epsilon \phi_{1}$, where $r_{0}$ is the radius function in Eq. (182), i.e. the solution of the boundary-value problem for the un-buckled plate. Inserting these into Eq. (190), we have

$$
\begin{aligned}
& \frac{d}{d R}\left(\begin{array}{c}
r_{0} \\
0 \\
r_{0}^{\prime} \\
0
\end{array}\right)+\epsilon \frac{d}{d R}\left(\begin{array}{c}
r_{1} \\
\phi_{1} \\
r_{1}^{\prime} \\
\phi_{1}^{\prime}
\end{array}\right) \\
& =\left.A^{-1} \mathbf{f}\right|_{\substack{r=r_{0}, r^{\prime}=r_{0}^{\prime} \\
\phi=0, \phi^{\prime}=0}}+\left.\epsilon \frac{\partial A^{-1} \mathbf{f}}{\partial\left(r, \phi, r^{\prime}, \phi\right)}\right|_{\substack{r=r_{0}, r^{\prime}=r_{0}^{\prime} \\
\phi=0, \phi^{\prime}=0}} \cdot\left(\begin{array}{c}
r_{1} \\
\phi_{1} \\
r_{1}^{\prime} \\
\phi_{1}^{\prime}
\end{array}\right)+\mathcal{O}\left(\epsilon^{2}\right) .
\end{aligned}
$$

Equating terms with equal powers of $\epsilon$, we are left with a system of linear equations for $\left(r_{1}, \phi_{1}, r_{1}^{\prime}, \phi_{1}^{\prime}\right)$ :

$$
\frac{d}{d R}\left(\begin{array}{c}
r_{1} \\
\phi_{1} \\
r_{1}^{\prime} \\
\phi_{1}^{\prime}
\end{array}\right)=\left.\frac{\partial A^{-1} \mathbf{f}}{\partial\left(r, \phi, r^{\prime}, \phi\right)}\right|_{\substack{r=r_{0}, r^{\prime}=r_{0}^{\prime} \\
\phi=0, \phi^{\prime}=0}} \cdot\left(\begin{array}{c}
r_{1} \\
\phi_{1} \\
r_{1}^{\prime} \\
\phi_{1}^{\prime}
\end{array}\right) .
$$




\subsection{Linear Boundary Conditions for Bifurcation}

We emply first-order expansions of the scalar coefficient of the radial stress in Eq. (168):

$$
\begin{aligned}
\frac{1}{\mu} \int_{-H}^{H}\left(\boldsymbol{a} \cdot \boldsymbol{P}_{a c t} \cdot \boldsymbol{h}_{1}\right) d Z & =\left.\left.\frac{1}{\mu} \int_{-H}^{H}\left(\boldsymbol{a} \cdot \boldsymbol{P}_{a c t} \cdot \boldsymbol{h}_{1}\right) d Z\right|_{\substack{r=r_{0}, r^{\prime}=r_{0}^{\prime} \\
\phi=0, \phi^{\prime}=0}}\right|_{\substack{r=r_{0}, r^{\prime}=r_{0}^{\prime} \\
\phi=0, \phi^{\prime}=0}} \cdot \epsilon\left(\begin{array}{c}
r_{1} \\
\phi_{1} \\
r_{1}^{\prime} \\
\phi_{1}^{\prime}
\end{array}\right)+\mathcal{O}\left(\epsilon^{2}\right) \\
& +\frac{\left.\left.\partial\left(\frac{1}{\mu} \int_{-H}^{H}\left(\boldsymbol{a} \cdot \boldsymbol{P}_{a c t} \cdot \boldsymbol{h}_{1}\right) d Z\right)\right|^{\prime}, \phi, r^{\prime}, \phi^{\prime}\right)}{} \\
& =2 H\left(\frac{\gamma_{2}}{\gamma_{1}} r_{0}^{\prime}+\kappa \frac{r_{0}}{R}-\frac{(1+\kappa) \gamma_{1} \gamma_{2}}{r_{0}^{\prime}}\right) \\
& +\epsilon\left(\frac{2 H \kappa}{R} r_{1}+\left(\frac{2 H \gamma_{2}}{\gamma_{1}}+\frac{2 H(1+\kappa) \gamma_{1} \gamma_{2}}{\left(r_{0}^{\prime}\right)^{2}}\right) r_{1}^{\prime}\right)+\mathcal{O}\left(\epsilon^{2}\right) .
\end{aligned}
$$

At $R=R_{0}$ and $R=R_{1}$, the radial stress of the flat annulus is zero, so for $i=0,1$,

$\left.\frac{1}{\mu} \int_{-H}^{H}\left(\boldsymbol{a} \cdot \boldsymbol{P}_{a c t} \cdot \boldsymbol{h}_{1}\right) d Z\right|_{R=R_{i}}=\left.\epsilon\left(\frac{2 H \kappa}{R} r_{1}+\left(\frac{2 H \gamma_{2}}{\gamma_{1}}+\frac{2 H(1+\kappa) \gamma_{1} \gamma_{2}}{\left(r_{0}^{\prime}\right)^{2}}\right) r_{1}^{\prime}\right)\right|_{R=R_{i}}+\mathcal{O}\left(\epsilon^{2}\right)$.

This provides one linear boundary condition for $R=R_{0}$ and one for $R=R_{1}$ :

$$
\frac{\kappa}{R} r_{1}+\left.\left(\frac{\gamma_{2}}{\gamma_{1}}+\frac{(1+\kappa) \gamma_{1} \gamma_{2}}{\left(r_{0}^{\prime}\right)^{2}}\right) r_{1}^{\prime}\right|_{R=R_{i}}=0
$$

We perform the same expansion procedure for the moment in Eq. (170):

$$
\begin{aligned}
\frac{1}{\mu} \int_{-H}^{H}\left(\boldsymbol{a} \cdot \boldsymbol{P}_{a c t} \cdot \boldsymbol{h}_{1}\right) Z d Z & =\left.\left.\frac{1}{\mu} \int_{-H}^{H}\left(\boldsymbol{a} \cdot \boldsymbol{P}_{a c t} \cdot \boldsymbol{h}_{1}\right) Z d Z\right|_{\substack{r=r_{0}, r^{\prime}=r_{0}^{\prime} \\
\phi=0, \phi^{\prime}=0}}\right|_{\substack{r=r_{0}, r^{\prime}=r_{0}^{\prime} \\
\phi=0, \phi^{\prime}=0}} \cdot \epsilon\left(\begin{array}{c}
r_{1} \\
\phi_{1} \\
r_{1}^{\prime} \\
\phi_{1}^{\prime}
\end{array}\right)+\mathcal{O}\left(\epsilon^{2}\right) \\
& +\frac{\left.\left.\partial\left(\frac{1}{\mu} \int_{-H}^{H}\left(\boldsymbol{a} \cdot \boldsymbol{P}_{a c t} \cdot \boldsymbol{h}_{1}\right) Z d Z\right)\right|^{\prime}, \phi, r^{\prime}, \phi^{\prime}\right)}{(196)} \\
& =0+\frac{2 H^{3} \epsilon}{3}\left(\frac{\kappa}{R} \phi_{1}+\left(\frac{\gamma_{2}}{\gamma_{1}}+\frac{(1+\kappa) \gamma_{1} \gamma_{2}}{\left(r_{0}^{\prime}\right)^{2}}\right) \phi_{1}^{\prime}\right)+\mathcal{O}\left(\epsilon^{2}\right) .
\end{aligned}
$$

Recall that the un-buckled plate has identically zero moment. This provides linear boundary conditions at $R=R_{0}$ and $R=R_{1}$ :

$$
\frac{\kappa}{R} \phi_{1}+\left.\left(\frac{\gamma_{2}}{\gamma_{1}}+\frac{(1+\kappa) \gamma_{1} \gamma_{2}}{\left(r_{0}^{\prime}\right)^{2}}\right) \phi_{1}^{\prime}\right|_{R=R_{i}}=0
$$

\subsection{Evidence of Bifurcation}

We now have a system of four linear ordinary differential equations and four linear boundary conditions. The linear boundary conditions at $R=R_{0}$ have a two-dimensional vector space 
of solutions. Considering that the boundary conditions at $R=R_{0}$ can be expressed as

$$
\left.\left(\begin{array}{cccc}
\frac{\kappa}{R} & \frac{\gamma_{2}}{\gamma_{1}}+\frac{(1+\kappa) \gamma_{1} \gamma_{2}}{\left(r_{0}^{\prime}\right)^{2}} & 0 & 0 \\
0 & 0 & \frac{\kappa}{R} & \frac{\gamma_{2}}{\gamma_{1}}+\frac{(1+\kappa) \gamma_{1} \gamma_{2}}{\left(r_{0}^{\prime}\right)^{2}}
\end{array}\right)\left(\begin{array}{c}
r_{1} \\
r_{1}^{\prime} \\
\phi_{1}^{\prime} \\
\phi_{1}^{\prime}
\end{array}\right)\right|_{R=R_{0}}=\left(\begin{array}{l}
0 \\
0
\end{array}\right)
$$

we see that the two-dimensional solution space at $R=R_{0}$ is spanned by, for example,

$$
\left.\left(\begin{array}{c}
r_{1} \\
r_{1}^{\prime} \\
\phi_{1}^{\prime} \\
\phi_{1}^{\prime}
\end{array}\right)\right|_{R=R_{0}}=\left.\left(\begin{array}{c}
\frac{\gamma_{2}}{\gamma_{1}}+\frac{(1+\kappa) \gamma_{1} \gamma_{2}}{\left(r_{0}^{\prime}\right)^{2}} \\
-\frac{\kappa}{R} \\
0 \\
0
\end{array}\right)\right|_{R=R_{0}} \text { and }\left.\left(\begin{array}{c}
r_{1} \\
r_{1}^{\prime} \\
\phi_{1}^{\prime} \\
\phi_{1}^{\prime}
\end{array}\right)\right|_{R=R_{0}}=\left.\left(\begin{array}{c}
0 \\
0 \\
\frac{\gamma_{2}}{\gamma_{1}}+\frac{(1+\kappa) \gamma_{1} \gamma_{2}}{\left(\frac{\kappa_{0}^{\prime}}{R}\right.} \\
-\frac{\kappa}{R}
\end{array}\right)\right|_{R=R_{0}}
$$

Let $\mathbf{u}$ and $\mathbf{v}$ be solutions of Eq. (192) with the initial $\left(R=R_{0}\right)$ conditions above. Then $\mathbf{u}$ and $\mathbf{v}$ have the forms

$$
\left(\begin{array}{l}
u_{1} \\
u_{2} \\
u_{3} \\
u_{4}
\end{array}\right)=\left(\begin{array}{c}
r_{1} \\
r_{1}^{\prime} \\
0 \\
0
\end{array}\right) \quad \text { and } \quad\left(\begin{array}{c}
v_{1} \\
v_{2} \\
v_{3} \\
v_{4}
\end{array}\right)=\left(\begin{array}{c}
0 \\
0 \\
\phi_{1} \\
\phi_{1}^{\prime}
\end{array}\right) .
$$

Every linear combination $\alpha \mathbf{u}+\beta \mathbf{v}$ is a solution of the differential equations that satisfies the linear boundary conditions Eq. (195) and (197) at $R=R_{0}$.

We seek a linear combination of this form that also satisfies the linear boundary conditions at $R=R_{1}$. That is, we want

$$
\left.\left(\begin{array}{cccc}
\frac{\kappa}{R} & \frac{\gamma_{2}}{\gamma_{1}}+\frac{(1+\kappa) \gamma_{1} \gamma_{2}}{\left(r_{0}^{\prime}\right)^{2}} & 0 & 0 \\
0 & 0 & \frac{\kappa}{R} & \frac{\gamma_{2}}{\gamma_{1}}+\frac{(1+\kappa) \gamma_{1} \gamma_{2}}{\left(r_{0}^{\prime}\right)^{2}}
\end{array}\right)\left(\begin{array}{l}
\alpha u_{1} \\
\alpha u_{2} \\
\beta v_{3} \\
\beta v_{4}
\end{array}\right)\right|_{R=R_{1}}=\left(\begin{array}{l}
0 \\
0
\end{array}\right) .
$$

We can seek the existence of such a linear combination by re-writing this condition as

$$
\left.\left(\begin{array}{cc}
\frac{\kappa}{R} u_{1}+\left(\frac{\gamma_{2}}{\gamma_{1}}+\frac{(1+\kappa) \gamma_{1} \gamma_{2}}{\left(r_{0}^{\prime}\right)^{2}}\right) u_{2} & 0 \\
0 & \frac{\kappa}{R} v_{1}+\left(\frac{\gamma_{2}}{\gamma_{1}}+\frac{(1+\kappa) \gamma_{1} \gamma_{2}}{\left(r_{0}^{\prime}\right)^{2}}\right) v_{2}
\end{array}\right)\right|_{R=R_{1}}\left(\begin{array}{l}
\alpha \\
\beta
\end{array}\right)=\left(\begin{array}{l}
0 \\
0
\end{array}\right)
$$

If the matrix has zero determinant, then there is a non-trivial solution $(\alpha, \beta)^{T}$ of this pair of linear equations at $R=R_{1}$.

We know that if $\gamma_{1}=\gamma_{2}$, then the growth tensor is a true deformation gradient, and there is no residual stress in the un-buckled annulus. We fix a value for $\gamma_{2}$ and let $\gamma_{1}$ vary until the determinant of the matrix in Eq. (202) is zero. In truth, we seek the first $\gamma_{1}$-value at which either of the non-zero entries in the matrix is zero. 


\subsection{Numerical Results}

In all numerical results found so far, the first term to drop to zero is the $(2,2)$-entry of the matrix in Eq. (202). This means that we can choose a perturbation of the moment alone. Further, the $(2,2)$-entry reaches zero at some $\gamma_{1}>\gamma_{2}$, which indicates buckled solutions for the case in which incompatible growth corresponds to azimuthal contraction.

In the following example, we set $\gamma_{2}=1.01, H=0.01, R_{0}=0.01, R_{1}=1$, and $\kappa=1$. As seen in Figures 8 and 9 , as functions of $\gamma_{1}$, the stress perturbation (dependent on $r_{1}$ alone) at $R=R_{1}$ has no zeros, while the moment perturbation (dependent on $\phi_{1}$ alone) at $R=R_{1}$ has multiple zeros, all greater than $\gamma_{2}$. The first four zeros are found at $\gamma_{1}=1.01086$, 1.01348, 1.01793, and 1.0242.

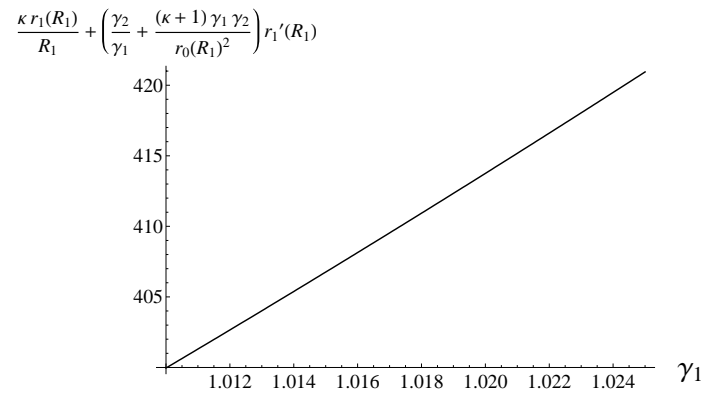

Figure 8: The stress perturbation (dependent on $r_{1}$ ) at $R=R_{1}$ has no zeros.

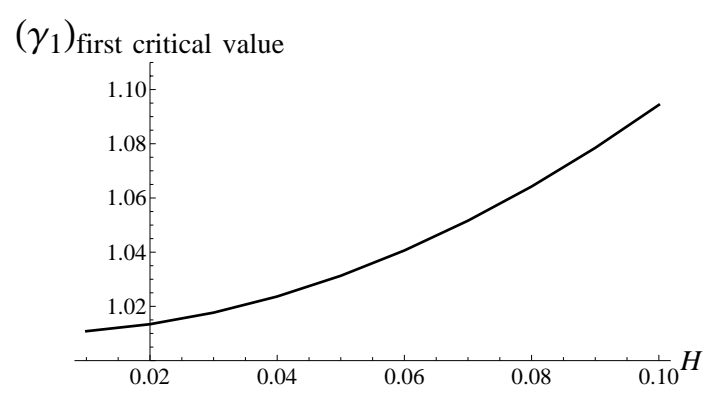

Figure 10: The first bifurcation value of $\gamma_{1}$, as a function of half-thickness $H$

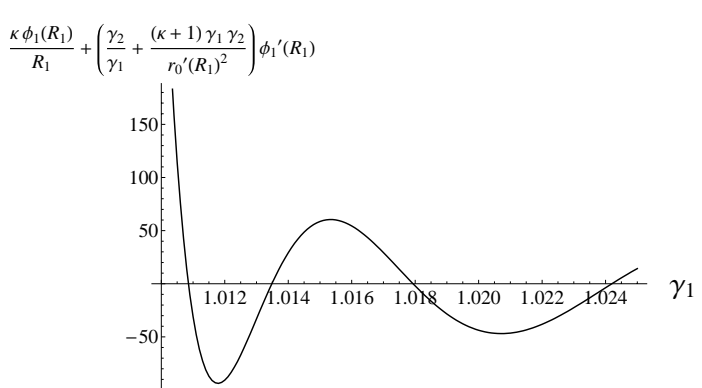

Figure 9: The moment perturbation (dependent on $\left.\phi_{1}\right)$ at $R=R_{1}$ has multiple zeros.

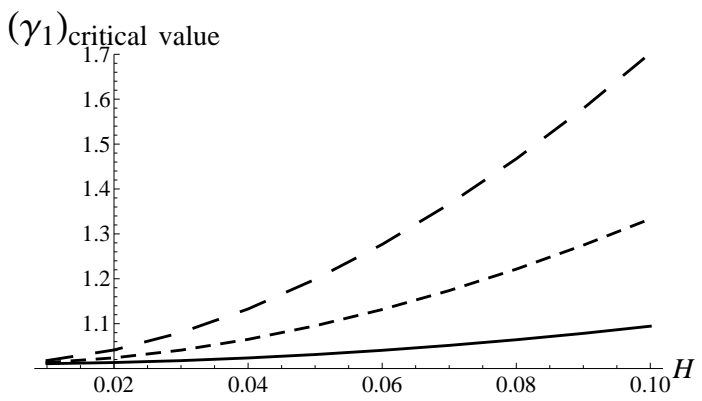

Figure 11: The first three critical values of $\gamma_{1}$, as functions of $H$

Figures 10 and 11 show that an increase in the half-thickness $H$ of the plate causes a delay in the onset of buckling. Figures 12-15 show the values of the buckling angle $\phi$, the middle surface height $\zeta$, and the thickness-integrated radial and azimuthal stresses and radial and azimuthal moments for $\gamma_{1}=56026090 / 55424431 \approx 1.01086$, which is very near a bifurcation value. in these figures, quantities computed with solutions of the perturbation equations are plotted with dashed lines, and those computed with solutions of the full nonlinear equations are plotted with solid lines. Since $\gamma_{1}$ is so close to the bifurcation value, the buckling is very small. 


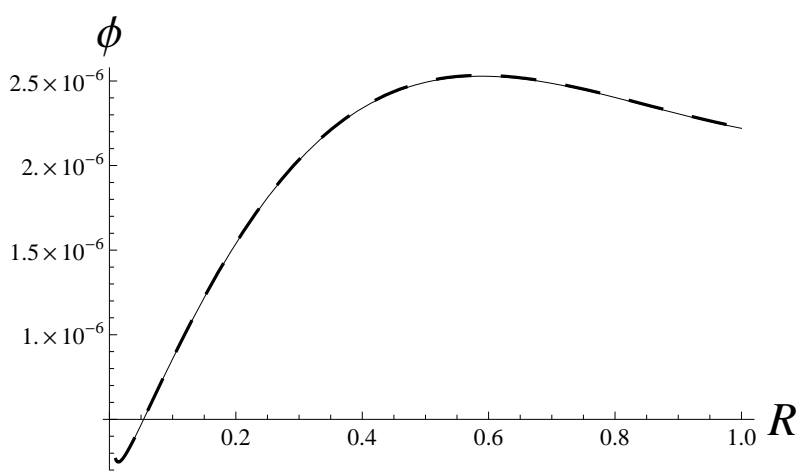

Figure 12: The buckling angle $\phi$ as a function of $R$.

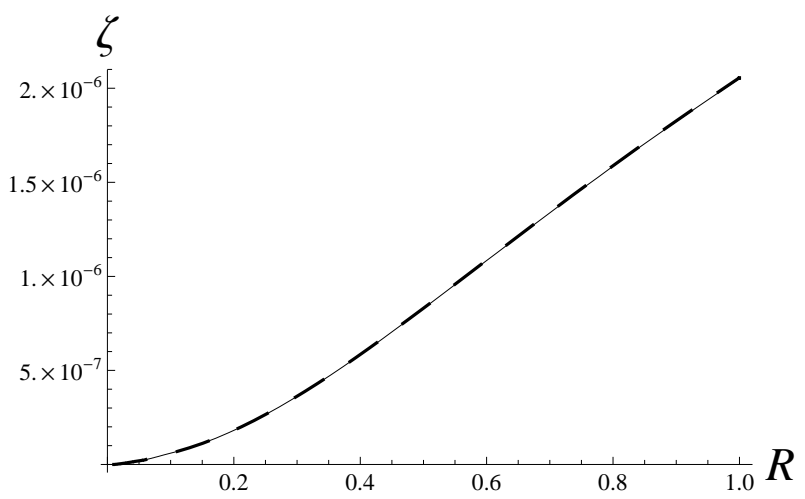

Figure 13: The height $\zeta$ of the middle surface as a function of $R$.

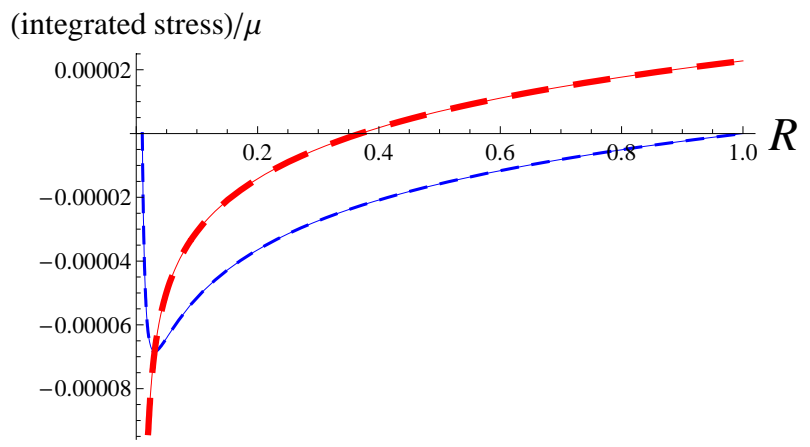

Figure 14: Radial (rapid dashed) and azimuthal (slower dashes) stresses as function of $R$. The results of perturbation and of solving the full nonlinear system of equations are overlaid.

\section{$9 \quad$ Large Buckling Due to Immersibility-Precluding Growth}

We consider a growth field of the kind in Eq. (51):

$$
\begin{aligned}
\boldsymbol{G} & =\gamma_{1} \boldsymbol{e}_{r} \otimes \boldsymbol{E}^{R}+\frac{\gamma_{2} R}{\int_{0}^{R} \gamma_{1}(s) d s} \boldsymbol{e}_{\theta} \otimes \boldsymbol{E}^{\Theta}+\boldsymbol{e}_{z} \otimes \boldsymbol{E}^{Z} \\
& =\gamma_{1} \boldsymbol{h}_{1} \otimes \boldsymbol{h}_{1}+\frac{\gamma_{2} R}{\int_{0}^{R} \gamma_{1}(s) d s} r \boldsymbol{h}_{2} \otimes \frac{\boldsymbol{h}_{2}}{R}+\boldsymbol{k} \otimes \boldsymbol{k} \\
& =\gamma_{1} \boldsymbol{h}_{1} \otimes \boldsymbol{h}_{1}+\gamma_{2} \boldsymbol{h}_{2} \otimes \boldsymbol{h}_{2}+\boldsymbol{k} \otimes \boldsymbol{k}
\end{aligned}
$$




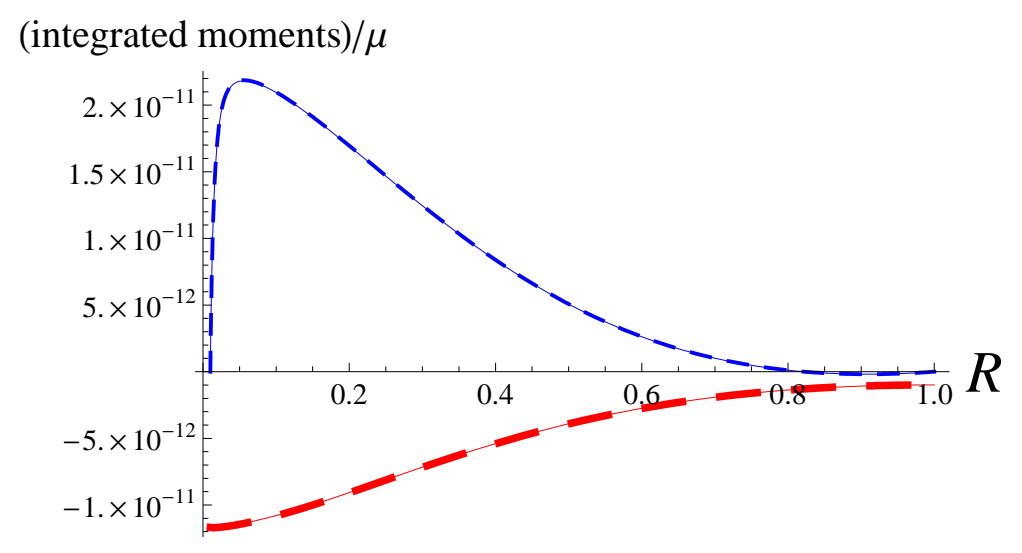

Figure 15: Radial (rapid dashes) and azimuthal (slower dashes) moments as function of $R$. The results of perturbation and of solving the full nonlinear system of equations are overlaid.

where $\gamma_{1}$ and $\gamma_{2}$ are continuously differentiable positive functions of $R$, and

$$
r(R)=\int_{0}^{R} \gamma_{1}(s) d s, \quad \theta(R, \Theta)=\frac{\gamma_{2}(R) R}{\int_{0}^{R} \gamma_{1}(s) d s} \Theta, \quad z=Z .
$$

This growth tensor is incorporated into the constitutive relation in the same fashion, and the differential equations are Eqs. (149) and (150), and the boundary conditions are Eqs. (153) and (154).

With parameters $R_{0}=0.2, R_{1}=0.709 H=0.01$, and $\kappa=1$, and growth factors

$$
\gamma_{1}(R) \equiv 1.9, \quad \gamma_{2}(R)=1.9-0.9 R^{2}
$$

we found a solution exhibiting significant buckling. Figures (16)-(19) show plots of the buckling angle, height of the buckled middle surface, stresses, and moments in the final configuration. The largest value of $\zeta$ is 0.064 , around $9 \%$ of the radius of the reference configuration. Buckling of this size is visible in Figure 20, which features a top view of the buckled middle surface.

\section{Conclusion}

We have demonstrated the relation of the standard kinematics of finite elasticity to the geometry of differentiable manifolds, and we have seen that kinematics can be adapted slightly to include incompatible growth. We have shown that incompatible growth is equivalent to a change of metric tensor on a Riemannian manifold such that the manifold can no longer be isometrically embedded in Euclidean three-dimensional space. We have also shown that the elastic response found in the multiplicative decomposition $\boldsymbol{F}=\boldsymbol{A} \cdot \boldsymbol{G}$ of the deformation gradient, amounts to a second change of metric, so that the manifold can again be isometrically embedded in $\mathbb{E}^{3}$. We have imported incompatible growth into geometrically exact models of plates and have solved models showing that incompatible growth alone can induce buckling, without the assistance of applied tractions. 


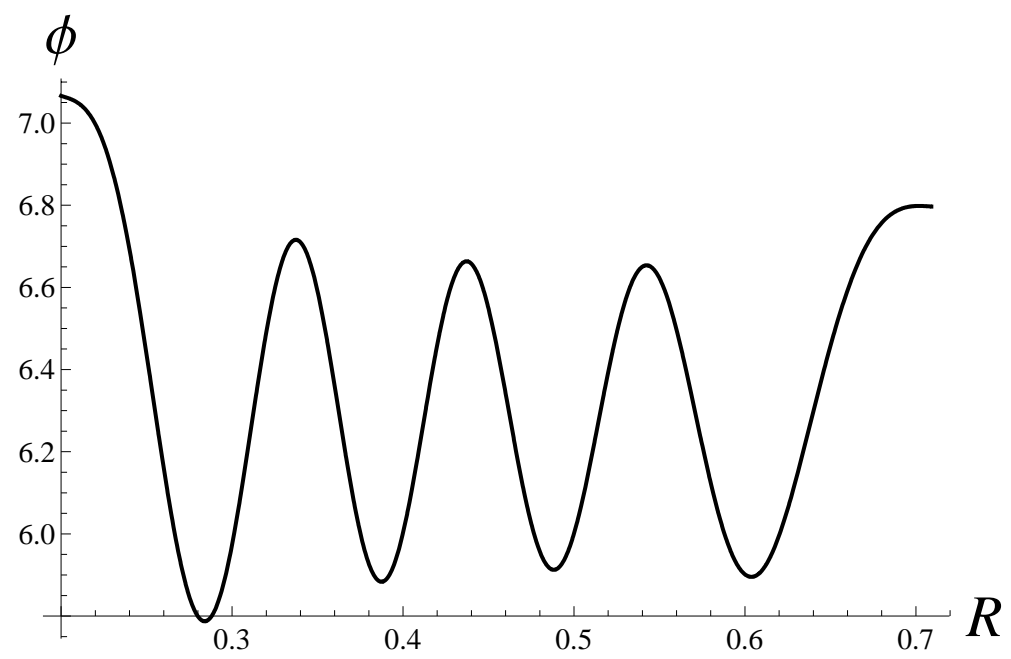

Figure 16: The buckling angle $\phi$ as a function of $R$.

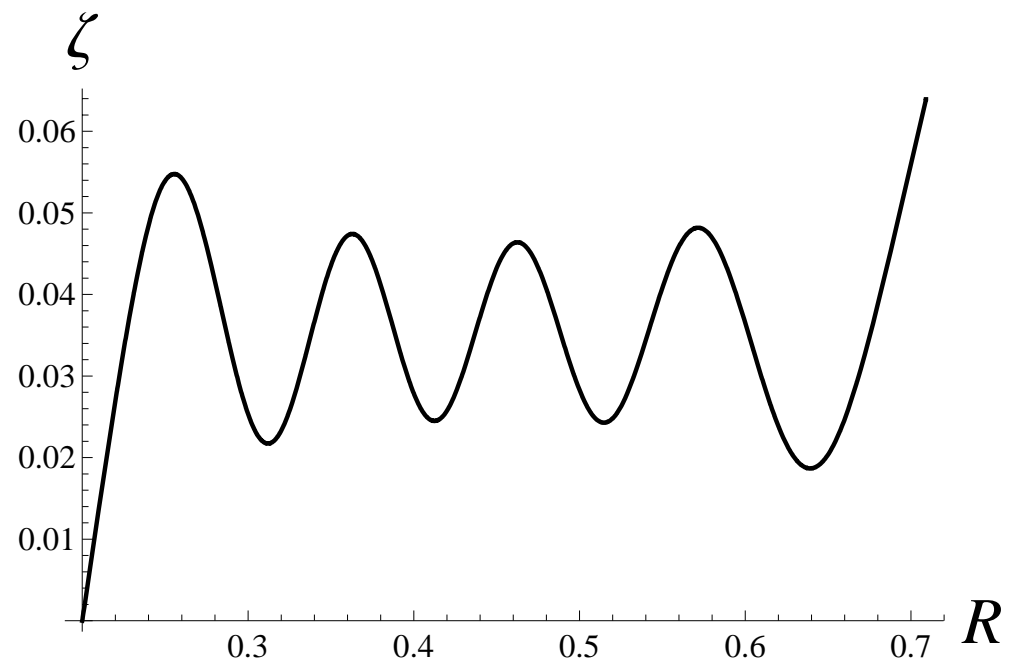

Figure 17: The height $\zeta$ of the middle surface as a function of $R$.

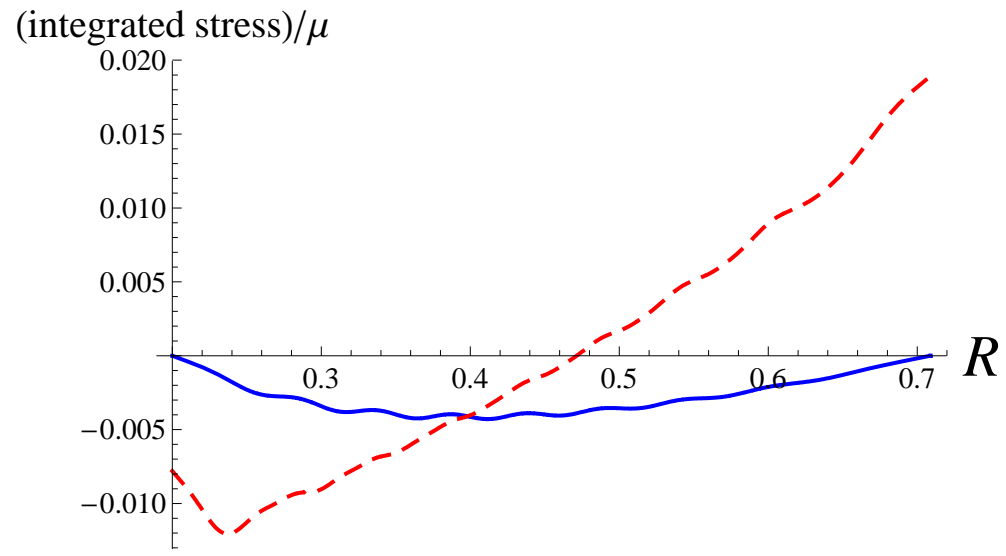

Figure 18: Radial (solid) and azimuthal (dashed) stresses as function of $R$ 


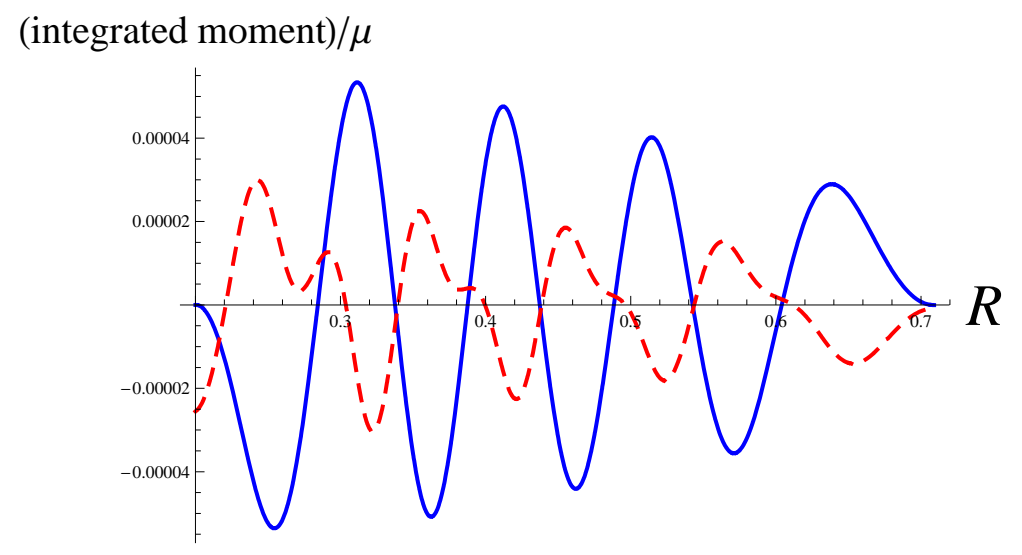

Figure 19: Radial (solid) and azimuthal (dashed) moments as function of $R$

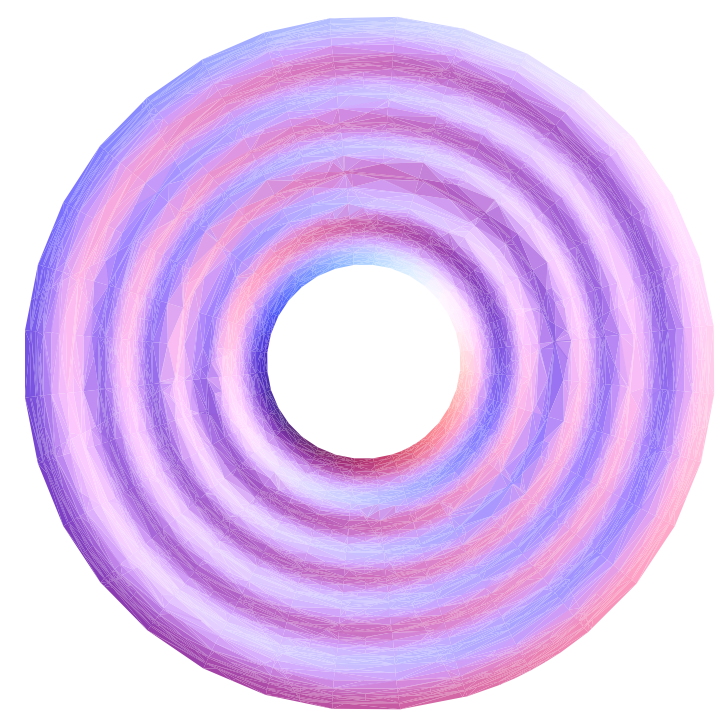

Figure 20: A top view of the buckled middle surface reveals buckling on the order of one-tenth the radius of the plate.

Acknowledgments: This publication is based on work supported by Award No. KUK-C1013-04, made by King Abdullah University of Science and Technology (KAUST), and based in part upon work supported by the National Science Foundation under grants DMS-0907773 $(\mathrm{AG})$ 


\section{References}

[1] S. S. Antman. Nonlinear Problems of Elasticity. Springer, Berlin, 1995.

[2] S. S. Antman and R. S. Marlow. Material constraints, Lagrange multipliers, and compatibility. Archive for Rational Mechanics and Analysis, 116:257-299, 1991.

[3] S. S. Antman and P. V. Negrón-Marrero. The remarkable nature of radially symmetric equilibrium states of nonlinearly elastic bodies. Journal of Elasticity, 18:131-164, 1987.

[4] M. Ben Amar and A. Goriely. Growth and instability in elastic tissues. J. Mech. Phys. Solids, 53:2284-2319, 2005.

[5] Y.-C. Chen and A. Hoger. Constitutive functions of elastic materials in finite growth and deformation. Journal of Elasticity, 59:173-193, 2000.

[6] P. G. Ciarlet. An Introduction to Differential Geometry with Applications to Elasticity. Springer Netherlands, 2005.

[7] J. Dervaux, P. Ciarletta, and M. Ben Amar. Morphogenesis of thin hyperelastic plates: A constitutive theory of biological growth in the Föppl-von Kármán limit. Journal of the Mechanics and Physics of Solids, 57(3):458-471, 2009.

[8] E. Efrati, E. Sharon, and R. Kupferman. Elastic theory of unconstrained non-Euclidean plates. Journal of the Mechanics and Physics of Solids, 57:762-775, 2009.

[9] A. Goriely and M. Ben Amar. Differential growth and instability in elastic shells. Phys. Rev. Lett., 94(19):198103, 2005.

[10] R. S. Marlow. Global material constraints and their associated reactive stresses. Journal of Elasticity, 33:203-212, 1993.

[11] J. McMahon. Geometry and Mechanics of Growing, Nonlinearly Elastic Plates and Membranes. PhD thesis, The University of Arizona, Tucson, Arizona, 2009.

[12] J. McMahon, A. Goriely, and M. Tabor. Growing nonlinearly elastic plates I: the genesis of residual stress. Preprint, 2010.

[13] R. W. Ogden. Non-Linear Elastic Deformations. Dover, Mineola, NY, 1984.

[14] E. K. Rodriguez, A. Hoger, and A. D. McCulloch. Stress-dependent finite growth law in soft elastic tissue. Journal of Biomechanics, 27:455-467, 1994. 


\section{RECENT REPORTS}

37/09 Optimal L2-error estimates for the semidiscrete Galerkin approximation to a second order linear parabolic initial and boundary value problem with nonsmooth initial data

Goswami

Pani

Goswami

Pani

Yadav

Kilpatrick

Bressloff neuronal network with synaptic depression

Kilpatrick

Bressloff synaptic depression

41/09 Homogenization for advection-diffusion in a perforated domain

Haynes

Hoang

Norris

Zygalakis

42/09 Fast stochastic simulation of biochemical reaction systems by alternative formulations of the Chemical Langevin Equation

Melykuti

Burrage

Zygalakis

Lazic

Finka et al.

Wright

Flyer

Yates

Baker

Erban

Maini

47/09 Stochastic Partial Differential Equations as priors in ensemble methods for solving inverse problems

Potsepaev

Farmer

Aziz

Cominetti et al.

Guazzelli

Hinch

02/10 Determining the equation of state of highly plasticised metals from Hinch

boundary velocimetry 
03/10 Stability of bumps in piecewise smooth neural Kilpatrick elds with nonlinear adaptation

Bressloff

04/10 Random intermittent search and the tug-of--war model of motor-

Newby driven transport

Bressloff

05/10 Ergodic directional switching in mobile insect groups

Escudero et al.

06/10 Derivation of a dual porosity model for the uptake of nutrients by root hairs

Zygalakis

Roose

07/10 Frost heave in compressible soils

Majumdar

Peppin

Style

Sander

08/10 A volume-preserving sharpening approach for the propagation of

Reis sharp phase boundaries in multiphase lattice Boltzmann simulations

09/10 Anticavitation and differential growth in elastic shells

Moulton

Goriely

10/10 On the mechanical stability of growing arteries

Goriely

Vandiver

11/10 Nonlinear Correction to the Euler Buckling Formula for Compressible Cylinders

De Pascalis

Destrade

Goriely

12/10 Nonlinear Morphoelastic Plates I: Genesis of Residual Stress

McMahon

Goriely

Tabor

Copies of these, and any other OCCAM reports can be obtained from:

Oxford Centre for Collaborative Applied Mathematics Mathematical Institute

24 - 29 St Giles'

Oxford

OX1 3LB

England

www.maths.ox.ac.uk/occam 